UNIVERSIDADE DE SÃO PAULO

INSTITUTO DE FÍSICA

\title{
PROBABILIDADE DE DETECÇÃO DE UM GAP DE RAPIDEZ NA PRODUÇÃO DO BOSON DE HIGGS
}

THAIS SCATTOLINI LORENA LUNGOV

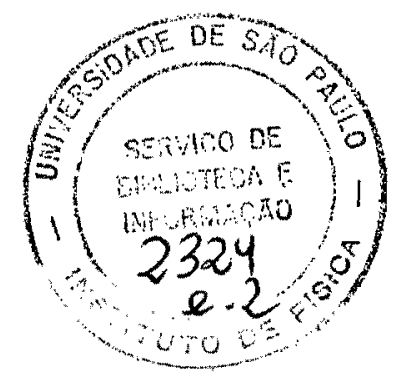

Tese de Doutoramento submetida ao Instituto de Física da Universidade de São Paulo

Prof. Dr. Carlos Ourivio Escobar (USP - Orientador)

Profa. Dra. Sandra dos Santos Padula (IFT-UNESP)

Prof. Dr. João Carlos dos Anjos (CBPF)

Prof. Dr. Adriano Antonio Natale (IFT-UNESP)

Prof. Dr. Marcelo Guzzo (UNICAMP)

São Paulo

11 de setembro de 1995
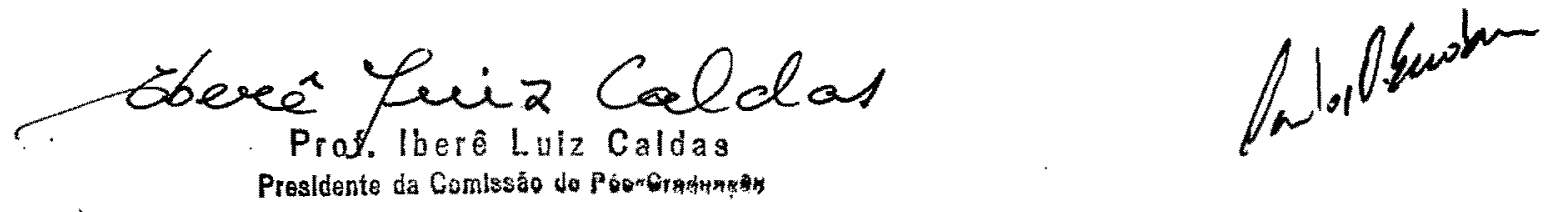

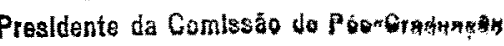

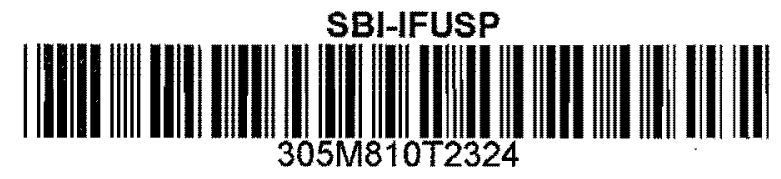




\section{FICHA CATALOGRÁFICA}

Preparada pelo Serviço de Biblioteca e Informação

do Instituto de Física da Universidade de São Paulo

Lungov, Thais Scattolini Lorena

Probabilidade de deteç̧ão de um gap de rapidez

na produção do boson de Higgs. São Paulo, 1995.

Tese (Doutoramento) - Universidade de São

Paulo. Instituto de Física. Departamento de Física Nuclear

Área de Concentração: Física das Partículas

Elementares

Orientador: Prof. Dr. Carlos Ourivio Escobar

Unitermos: 1. Espalhamento múltiplo; 2. Interações eletrofracas e fortes;3. Boson de Higgs; 4 . teoria de Regge; 5. Dualidade.

USP/IF/SBI - 43/95 
ao Wagner, aos meus filhos, aos meus pais. 
... all you touch and all you see is all your life will ever be. Roger Waters 


\section{AGRADECIMENTOS}

Agradeço ao Prof. Dr. Carlos Ourivio Escobar pela sugestão do tema, amizade, orientação e incentivo.

Ao Prof. Dr. Oscar José Pinto Éboli e à Prof. Dra. Renata Zukanovich Funchal pelas discussões, apoio ao trabalho e grande amizade.

A todos os amigos do Instituto, em especial à Rosana e à Maria Augusta pela amizade e longas conversas sobre física ou não.

À Gina, pela colaboração dada colocando à minha disposição os serviços da secretaria, muitas vezes além do seu horário de trabalho.

Ao Wagner, pelas figuras, e especialmente pela paciência em todos esses anos.

Aos meus filhos, Felipe, Paula e André pelo apoio e incentivo.

À FAPESP e ao CNPq pelo suporte financeiro. 


\section{RESUMO}

Foi analisada, através de simulação Monte Carlo, a probabilidade de que um gap de rapidez seja observado na produção do boson de Higgs por fusão de bosons vetoriais com massa (W), no LHC (Large Hadron Collider). Foi analisada, também através do uso de Monte Carlo, a possibilidade de que a existência ou não de um gap de rapidez seja usada na distinção de um higgs produzido por fusão de bosons vetoriais $W$ de outro produzido por fusão de gluons. Os diferentes modelos para tratamento de processos não perturbativos dos códigos Monte Carlo DTUJET e PYTHIA foram comparados. 


\begin{abstract}
The gap survival probability for a higgs boson produced by $W$ fusion at the LHC (Large Hadron Collider) is analysed through Monte Carlo simulation. The possibility of distinguishing between two higgs production mechanisms, $W$ and gluon fusion is studied using Monte Carlo simulation. The two different models for non perturbative processes, used by DTUJET and PYTHIA are compared.
\end{abstract}




\section{Índice}

1 Introdução 3

2 O Modelo Padrão 6

2.1 Quebra espontânea de Simetria . . . . . . . . . . . 8

2.2 Geração de Massa no Modelo Padrão . . . . . . . . . . . 10

2.3 A Invariância de Gauge . . . . . . . . . . . . . . . . . 16

2.4 Unitariedade. . . . . . . . . . . . . . . . . 21

2.5 Problemas com o Modelo Padrão . . . . . . . . . 22

3 Higgs 26

3.1 Determinação da Massa . . . . . . . . . . . . . 26

3.1.1 Correções Radiativas . . . . . . . . . . . . . 26

3.1 .2 Limites Experimentais . . . . . . . . . . . . . 29

3.1 .3 Limites Teóricos. . . . . . . . . . . . . . . . . . . 29

3.2 Modos de Decaimento . . . . . . . . . . . . . . 32

3.2.1 Teorema de Baixas Energias para Interações do Higgs . 33

3.2.2 Decaimento em Fermions . . . . . . . . . . . . . . 33

3.2 .3 Decaimento em Gluons . . . . . . . . . . . . . . 34

3.2 .4 Decaimento em Fotons . . . . . . . . . . . . 37

3.2 .5 Decaimento em $Z Z^{*}$ e $W^{*} W \ldots \ldots \ldots$. . . . . 39

3.2.6 Decaimento em $Z Z$ ou $W^{+} W^{-} \ldots \ldots \ldots \ldots$

3.3 Modos de Produção . . . . . . . . . . . . . . . . 41

3.3.1 Fusão de Fermions . . . . . . . . . . . . . . . . . 42

3.3 .2 Bremsstrahlung de $Z \ldots \ldots \ldots \ldots \ldots$

3.3.3 Bremsstrahlung de $W^{ \pm} \ldots \ldots \ldots \ldots \ldots$. . . . . 44

3.3.4 Fusăo de Gluons . . . . . . . . . . . . . . . 45

3.3.5 Fusão de W's ou de Z's . . . . . . . . . . . . . . . 46 
4 Colisões Hadrônicas $\quad \mathbf{5 1}$

4.1 Seção de Choque Total . . . . . . . . . . . . . . . 51

4.1.1 Processos Difrativos .............. . 52

4.1.2 Processos Não Difrativos .............. 57

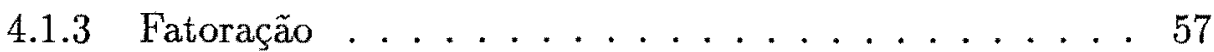

4.2 Geradores de Eventos . . . . . . . . . . . . . 60

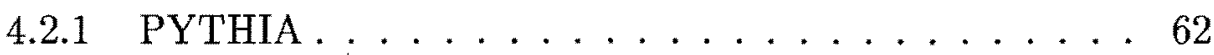

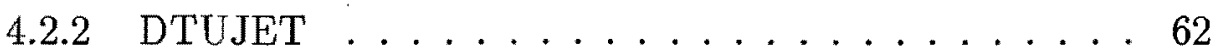

5 Gap de Rapidez $\quad \mathbf{7 0}$

5.1 Dificuldades na Deteç̧ão do Gap . . . . . . . . . . . 75

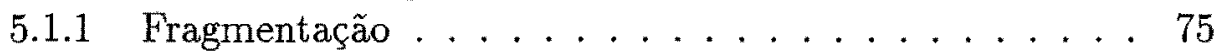

5.1 .2 Interações Múltiplas . . . . . . . . . . . . 77

5.1 .3 Pile Up .................... . . . 80

5.2 Probabilidade de Sobrevivência do Gap . . . . . . . . . . . 81

5.2.1 Cálculo de $\left\langle|S|^{2}\right\rangle \ldots \ldots . \ldots . \ldots 8$

6 Análise $\quad 87$

6.1 A Geração dos Eventos . . . . . . . . . . . . . . . . 88

6.1 .1 Algumas Informações . . . . . . . . . . . . . . . . 88

6.1.2 Seleção dos Eventos . . . . . . . . . . . . . 90

6.1.3 Processos Analisados ... . . . . . . . . . . 93

6.1.4 Cortes Adotados para Seleção dos Eventos . . . . . . 95

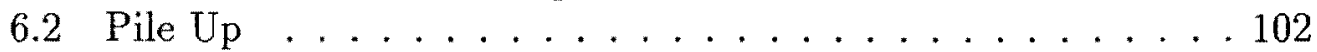

7 Resultados e Discussões $\quad 104$

7.1 Probabilidade de Sobrevivência do Gap . . . . . . . . . . . . . 104

7.2 Seção de Choque e Luminosidade . . . . . . . . . . . . . . 115

7.3 Comparando Dois Modelos de Minimum Bias . . . . . . . 125

8 Conclusão 


\section{Capítulo 1}

\section{Introdução}

No final da década de 70, a Física de Altas Energias já possuía estruturados os modelos que a descrevem até os dias de hoje. O modelo de Weinberg-SalamGlashow já havia sido desenvolvido, bem como a QCD (Cromodinâmica Quântica), ambas baseadas em teoria de campos de gauge, e renormalizáveis. No início da década de 80 , todas as partículas previstas pelo modelo, com exeção do quark top, do $\nu_{\tau}$ e do higgs, já haviam sido observadas em laboratório. Desde então, diversas áreas têm se desenvolvido, mas nenhuma modificação fundamental foi inserida. A física de correções radiativas desenvolveu-se bastante, fato que permitiu que sejam feitos testes de precisão do modelo, sempre com grande concordância.

Recentemente, a observação do quark top no FERMILAB, trouxe mais uma confirmação para o modelo padrão, completando a terceira geração de fermions (há evidências indiretas da existência do $\nu_{\tau}$ ).

É certo, todavia, que o modelo padrão não é uma teoria final, pois deixa de responder muitas perguntas fundamentais. No entanto, com as energias atualmente disponíveis nos aceleradores, não foi possível encontrar nem desvios desse modelo, e nem indicações de como seria um novo modelo, ou mais abrangente, ou que o substituísse.

Há diversos aspectos acerca dos quais não foi possível obter informações, sendo o principal deles o setor de quebra de simetria. Não existe evidência experimental até o momento para o boson de Higgs. Todos os motivos para que ele exista são teóricos. Por outro lado, não há tampouco informação experimental que indique uma nova direção a seguir. Um importante detalhe que surgiu recentemente com a observação do top, e que talvez seja sinal de 
que pode haver física nova, é o fato de sua massa ser muito grande. Como as interações dos fermions com o higgs são proporcionais à massa do fermion, o top deve interagir fortemente com o higgs, o que não ocorre com nenhum dos outros quarks.

A deteção do higgs, assim como o estudo detalhado do setor de quebra de simetria devem trazer informações sobre a física que engloba o modelo padrão. Torna-se portanto necessária por exemplo, a determinação correta do acoplamento do higgs com cada uma das outras partículas com que ele interage, e especialmente com os bosons longitudinais.

Em 1986 Khoze, Dokshitzer e Troyan propuseram uma forma de distinguir o sinal de um higgs produzido pela fusão de W's de outro produzido pela fusão de gluons, baseada na diferença entre os fluxos de cor desses dois processos.

Gluons emitem radiação, que posteriormente se transforma em hadrons produzidos na região central de rapidez; o mesmo não ocorre para os bosons W (nem para o Z), de forma que uma fusão de W's produzindo o higgs conduz a. um evento sem hadrons na região central do detetor, ou seja com um gap de rapidez.

Porém diversos obstáculos precisam ser contornados antes que tal característica possa ser aproveitada. Em primeiro lugar as seções de choque envolvidas são muito pequenas, de forma que mesmo em anéis de colisão com grande energía no centro de massa serão produzidos poucos eventos por ano. É necessário por isso, que a diferença entre os processos seja muito clara, para que venha a ser útil. Mas infelizmente não é o que ocorre. As interações que ocorrem entre as partículas que restam no feixe incidente após a interação dura contaminam o gap com grande freqüência. Pior que isso, essas interações envolvem pequena troca de momento transversal, de forma que não podem ser tratadas perturbativamente. Existem diversos modelos que descrevem essa física, em perfeito acordo com os dados atualmente disponíveis, porém partindo de princípios diferentes e algumas vezes inconciliáveis, e com diferentes previsões para energias mais altas que as já testadas até o momento.

Os objetivos desse trabalho são:

- Verificar de forma ampla e detalhada a possibilidade da detecção experimental de um eventual gap de rapidez ocorrido num evento de produção de higgs por fusão de bosons vetoriais com massa ${ }^{1}$ num acelerador

\footnotetext{
${ }^{1}$ Será considerado somente o higgs descrito pelo modelo padrão.
} 
como o LHC (Large Hadron Collider), atualmente em construção no CERN.

- Verificar a possibilidade de que a existência ou não de gap de rapidez seja usada na distinção dos dois modos de produção do higgs mencionados (fusão de gluons e fusão de bosons vetoriais com massa) no LHC.

- Comparar os resultados de dois geradores Monte Carlo que se baseiam em modelos diferentes para as interações secundárias, compostas da fragmentação das partículas remanescentes dos feixes, das colisões duras e soft que ocorrem além da colisão dura principal e dos processos difrativos. Os dois modelos utilizados, o DTUJET e o PYTHIA estão escritos na forma de geradores de eventos Monte Carlo. O PYTHIA não é um modelo em si, mas sim uma compilação de diferentes modelos usados em diferentes situações. Essa observação ficará clara no decorrer da leitura da tese.

O trabalho consiste basicamente de geração de eventos por método Monte Carlo, e subseqüente análise. A tese está dividida da seguinte maneira. No cap. 2 é feita uma apresentação geral do Modelo Padrão, com ênfase na teoria eletrofraca e uma breve discussão sobre alguns de seus problemas. O cap.3 é dedicado ao estudo do higgs, analisando com certo detalhe seus modos de produção e decaimento principais. O cap.4 apresenta alguns conceitos fundamentais da teoria de hadrons, e a forma como as interações hadrônicas são tratadas nos dois modelos utilizados no trabalho, o DTUJET e o PYTHIA. No cap.5 é apresentada a idéia de gap de rapidez e são discutidas as dificuldades que devem advir da tentativa de utilização dessa característica na distinção dos modos de produção do higgs. Aqui, é aprofundada a análise das diferenças entre os dois geradores de eventos. No cap. 6 são apresentados os métodos utilizados na geração e análise dos eventos. Finalmente, no cap.7 são apresentados e discutidos os resultados obtidos com a simulação. $\mathrm{O}$ cap.8 apresenta algumas conclusões finais.

É utilizado, em todo o trabalho, o Sistema Natural de Unidades, em que $\hbar=c=1$. Sendo assim, massa, energia e momento possuem todos a mesma unidade, que na maior parte das vezes será o $\mathrm{GeV}$. 


\section{Capítulo 2}

\section{O Modelo Padrão}

As partículas fundamentais conhecidas atualmente agrupam-se, de acordo com as teorias atualmente consideradas (Modelo de Weinberg-Salam e QCD) da seguinte forma:

- bosons de gauge - são partículas que mediam as interações: fotons, $W^{+}, W^{-}, Z_{0}$, gluons (8).

- fermions - quarks e leptons, que estão distribuídos em 3 gerações.

A tabela (2) mostra os fermions conhecidos agrupados em três gerações, conforme o Modelo Padrão.

O quark top foi detetado recentemente, preenchendo a lacuna que havia no setor de quarks da terceira geração. A única partícula dessa tabela que resta sem haver sido observada é o neutrino do tau $\left(\nu_{\tau}\right)$, mas existem diversas evidências indiretas de sua existência (principalmente devido à conservação do número leptônico e à cinemática dos decaimentos do $\tau$.).

As interações são descritas através de uma teoria de gauge baseada no grupo $S U(3)_{C} \otimes S U(2)_{L} \otimes U(1)_{Y}$.

A QCD (Quantum Chromodynamics) é uma teoria de gauge renormalizável, que usa o grupo de simetria $S U(3)_{C}$ para descrever as interações fortes entre os quarks, sendo os gluons os bosons vetoriais mediadores dessas interações.

Cada um dos quarks existe em três cores; a cor é um número quântico introduzido para resolver alguns problemas como:

- anomalia quiral: o Modelo Padrão é uma teoria quiral porque os bosons de gauge acoplam-se de forma diferente com os fermions de mão direita e 


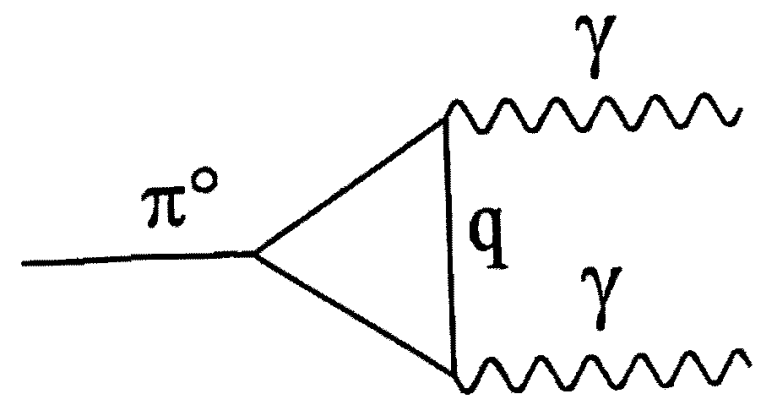

Figura 2.1: Exemplo de diagrama de triângulo que dá origem à anomalia quiral.

\begin{tabular}{ccrcc} 
& & \multicolumn{3}{c}{ massa } \\
nome & símbolo & carga & $(\mathrm{GeV})$ & tipo \\
up & $u$ & $\frac{2}{3}$ & $4 \times 10^{-3}$ & quark \\
down & $d$ & $-\frac{1}{3}$ & $7 \times 10^{-3}$ & quark \\
$\begin{array}{c}\text { - neutrino } \\
\text { electron }\end{array}$ & $\nu_{e}$ & 0 & $<1.8 \times 10^{-8}$ & lepton \\
charm & $c$ & -1 & $5.1 \times 10^{-4}$ & lepton \\
strange & $s$ & $-\frac{1}{3}$ & 1.5 & quark \\
$\mu-$ neutrino & $\nu_{\mu}$ & 0 & 0.2 & quark \\
muon & $\mu$ & -1 & 0.106 & lepton \\
top (truth) & $t$ & $\frac{2}{3}$ & 174 & quark \\
bottom (beauty) & $b$ & $-\frac{1}{3}$ & 4.7 & quark \\
$\tau-$ neutrino & $\nu_{\tau}$ & 0 & $<7 \times 10^{-2}$ & lepton \\
tau & $\tau$ & -1 & 1.78 & lepton
\end{tabular}

Tabela 2.1: Fermions Integrantes das três gerações conhecidas do Modelo Padrão. Tabela retirada da ref. [1] 
gauge parcial", que era quebrada explicitamente pelos termos de massa na Lagrangiana. Já eram conhecidas as correntes fracas carregadas e a eletromagnética, de forma que foram propostos três bosons para intermediar essas interações: $W^{+}, W^{-}$e $\gamma$. O fato dos bosons $W^{ \pm}$posssuírem massa, (o que ficava evidente a partir dos resultados experimentais), prejudicava muito a tentativa de analogia com a QED, que na época já era uma teoria bem estabelecida. Glashow verificou que a forma mais simples de introduzir um termo de massa para os W's na Lagrangiana era consider a existência de correntes fracas neutras, além das carregadas, passando portanto a considerar, além dos três bosons já mencionados, um outro neutro, o $Z_{0}$. Nessa época já haviam sido introduzidos os grupos $S U(2) \otimes U(1)$, e portanto o isospin fraco e a hipercarga fraca.

Vale notar que o termo de massa para os fermions também viola a invariância de gauge quando colocado diretamente na Lagrangiana. Um termo do tipo $m\left(\overline{\psi_{L}} \psi_{R}+\overline{\psi_{R}} \psi_{L}\right)$ não é invariante de gauge se os fermions de mão direita e mão esquerda se acoplam de forma diferente com os bosons de gauge. Se isso for modificado, colocando-se acoplamentos iguais para os fermions com as duas quiralidades, decorre a conservação de paridade, contrária à experiência.

Em 1967 Weinberg [3] e em 1968 Salam [4] propuseram (em trabalhos independentes), que a massa dos bosons $W^{ \pm}$e $Z_{0}$ fossem introduzidas através de uma quebra espontânea de simetria, ou seja, o termo de massa não seria colocado explicitamente na Lagrangiana, mas surgiria como resultado da assimetria do vácuo.

Esse mecanismo, conhecido como Mecanismo de Higgs, havia sido proposto em 1964 por P. Higgs [5] (e paralelamente por Englert e Brout [6]). Segundo ele, a quebra de simetria ocorre quando o Hamiltoniano e as relações de comutação de uma teoria apresentam uma simetria que não é acompanhada por seus estados físicos. No caso do Modelo Padrão, o vácuo não é invariante por uma transformação de gauge, embora o Hamiltoniano (e a Lagrangiana) o sejam. Esse vácuo não vazio gera massa para os bosons vetoriais.

Em 1967 Kibble [7] estendeu o mecanismo às teorias não abelianas, deixando o mecanismo de Higgs na forma como é usado atualmente no Modelo Padrão. Foi com esse trabalho de Kibble que Weinberg teve contato, associando-o à unificação das teoria fracas e eletromagnética, e à mistura dos bosons proposta por Glashow. 


\subsection{Geração de Massa no Modelo Padrão}

A Lagrangiana antes da quebra espontânea de simetria (em que nem os fermions nem os bosons possuem massa) é:

$$
\mathcal{L}=-\frac{1}{4} \vec{W}^{\mu \nu} \cdot \vec{W}_{\mu \nu}-\frac{1}{4} B^{\mu \nu} B_{\mu \nu}+\bar{\psi}_{i} \gamma^{\mu} D_{\mu} \psi_{i}
$$

onde

$$
\vec{W}^{\mu \nu}=\partial_{\mu} \vec{W}_{\nu}-\partial_{\nu} \vec{W}_{\mu}-g \vec{W}_{\mu} \times \vec{W}_{\nu}
$$

sendo $\vec{W}_{\mu}$ um isotripleto de gauge de Yang-Mills, que é o boson que carrega a interação $S U(2)_{L}$. Cada boson $\mathrm{W}$ possui somente duas polarizações transversais, porque não possuem massa.

$$
B_{\mu \nu}=\partial_{\mu} B_{\nu}-\partial_{\nu} B_{\mu}
$$

sendo $B_{\mu}$ o boson de gauge do grupo $U(1)_{Y}$, possuindo também dois modos de polarização transversais.

A derivada covariante é dada por:

$$
D_{\mu}=\partial_{\mu}+i g \vec{W}_{\mu} \cdot \vec{T}+i g^{\prime} \frac{1}{2} B_{\mu} Y
$$

sendo que

- $g$ é a constante de acoplamento de $S U(2)_{L}$;

- $g^{\prime}$ é a constante de acoplamento de $U(1)_{Y}$;

- $\vec{T}$ é o operador de isospin fraco;

- $Y$ é a hipercarga fraca.

Os bosons de gauge físicos $W^{+}, W^{-}, Z_{0}$ e $\gamma$ são obtidos a partir de misturas de $W_{1}, W_{2}, W_{3}$ e B:

$$
\begin{aligned}
& W^{+}=\frac{1}{\sqrt{2}}\left(W_{1}+i W_{2}\right) \\
& W^{-}=\frac{1}{\sqrt{2}}\left(W_{1}-i W_{2}\right)
\end{aligned}
$$




$$
\left(\begin{array}{c}
W_{3} \\
B
\end{array}\right)=\left(\begin{array}{cc}
\cos \theta_{W} & \sin \theta_{W} \\
-\sin \theta_{W} & \cos \theta_{W}
\end{array}\right)\left(\begin{array}{c}
Z \\
A
\end{array}\right)
$$

sendo que $A$ representa um foton, e $\theta_{W}$ é o ângulo de Weinberg, ou ângulo de mistura eletrofraca, um parâmetro da teoria. A partir de seu valor e do da carga elétrica, os acoplamentos de gauge estão determinados (como será visto adiante).

Após essa mistura, obtêm-se quatro campos de Yang-Mills com duas polarizações transversais (sem massa).

Impondo que o coeficiente de $A$ na mistura seja $i e Q=i e\left(T_{3}+\frac{1}{2} Y\right)$, são obtidas as relações:

$$
\begin{gathered}
g=\frac{e}{\sin \theta_{W}} \\
g^{\prime}=\frac{e}{\cos \theta_{W}} \\
\frac{1}{g^{2}}+\frac{1}{g^{\prime 2}}=\frac{1}{e^{2}}
\end{gathered}
$$

Usando esses campos físicos a derivada covariante fica:

$$
D=\partial+i e Q A+\frac{i}{\sqrt{2}} g\left(\tau^{+} W^{+}+\tau^{-} W^{-}\right)+i g_{Z}\left(\frac{1}{2} \tau_{3}-x_{W} Q\right) Z
$$

sendo $\tau_{i}=2 T$ as matrizes de Pauli, $\tau^{+}=\sqrt{2} T^{+}=\left(\begin{array}{ll}0 & 1 \\ 0 & 0\end{array}\right)$ e $\tau^{-}=\sqrt{2} T^{-}=$ $\left(\begin{array}{ll}0 & 0 \\ 1 & 0\end{array}\right)$, e também $g_{z}=\frac{g}{\cos \theta_{W}}$ e $x_{W}=\sin ^{2} \theta_{W}$,

A proposta de Weinberg e Salam para a geração das massas dos bosons começa com a introdução na Lagrangiana (1) do termo:

$$
\mathcal{L}_{\Phi}=\left(D_{\mu} \Phi\right)^{\dagger} D^{\mu} \Phi-V\left(\Phi^{\dagger} \Phi\right)
$$

com:

$$
V=-\mu^{2} \Phi^{\dagger} \Phi-\lambda\left(\Phi^{\dagger} \Phi\right)^{2}
$$

sendo $\Phi$ um isodubleto sob o grupo $S U(2)$, 


$$
\Phi=\left(\begin{array}{c}
\Phi^{+} \\
\Phi^{0}
\end{array}\right)=\exp \left(\frac{i \xi(x) \cdot \tau}{2 v}\right)\left(\begin{array}{c}
0 \\
\frac{v+h(x)}{\sqrt{2}}
\end{array}\right)
$$

onde $v$ é o valor esperado de $|\Phi|$. Os campos $\xi_{i}(x)$ e $h(x)$ são reais.

O potencial tem um mínimo para $\Phi^{*} \Phi=\frac{v^{2}}{2}$, onde $\frac{v}{\sqrt{2}}=\left(\frac{-\mu^{2}}{2 \lambda}\right)^{\frac{1}{2}}$, e portanto $\Phi$ adquire um valor esperado no vácuo (vev) $v$.

Pode-se fazer uma transformação de gauge que elimina os três campos $\xi_{i}(x)$ e deixa $H(x)$ com vev nulo. Esse gauge é conhecido como gauge unitário, e nele $\Phi$ assume a forma:

$$
\Phi(x)=\frac{1}{\sqrt{2}}\left(\begin{array}{c}
0 \\
v+h(x)
\end{array}\right)
$$

Os três campos desaparecidos são os três bosons de Goldstone. Porém como essa é uma teoria de gauge, os três bosons de Goldstone reaparecem como os modos longitudinais para os bosons $W^{+}, W^{-}$e $Z^{0}$, que assim adquirem massa. O campo remanescente, $h(x)$, é conhecido como boson de Higgs, que só pode ser um escalar, pois tem somente uma componente, e deve portanto ter spin zero.

Por esse motivo, estudar em laboratório as interações dos bosons $W^{+}, W^{-}$ e $Z^{0}$ longitudinalmente polarizados, deve trazer alguma luz ao mecanismo de quebra espontânea de simetria, ainda desconhecido.

De (2.12) e (2.15) obtém-se:

$$
\mathcal{L}_{\Phi}=\frac{1}{2}(\partial h)^{2}+\frac{1}{4} g^{2} W^{+} W^{-}(v+h)^{2}+\frac{1}{8} g_{Z}^{2} Z Z(v+h)^{2}-V\left[\frac{1}{2}(v+h)^{2}\right] .
$$

de forma que somente os bosons de gauge $W^{+}, W^{-}$e $Z_{0}$ acoplam-se com o higgs segundo os termos:

$$
\mathcal{L}_{\text {vet }}=\left(\frac{1}{4} g^{2} W^{+} W^{-}+\frac{1}{8} g_{Z}^{2} Z Z\right)\left(h^{2}+2 v h\right) .
$$

Da eq. (2.16), conclui-se que:

$$
M_{W}=\frac{1}{2} g v,
$$

e 


$$
\begin{aligned}
M_{Z} & =\frac{1}{2} \frac{v g}{\cos \theta_{W}}= \\
& =\frac{v}{2} \sqrt{g^{2}+g^{\prime 2}}= \\
& =\frac{M_{W}}{\cos \theta_{W}} .
\end{aligned}
$$

enquanto o foton permanece sem massa.

Desenvolvendo-se a Lagrangiana (2.16), obtêm-se os seguintes termos descrevendo o higgs:

$$
\mathcal{L}_{h}=\frac{1}{2}(\partial h)^{2}-\frac{1}{2}\left(-2 \mu^{2}\right) h^{2}+\frac{1}{4} \mu^{2} v^{2}\left[-1+\frac{4 h^{3}}{v^{3}}+\frac{h^{4}}{v^{4}}\right] .
$$

A massa para o boson escalar, como se pode observar diretamente do termo do potencial depende da constante de acoplamento $\lambda$, que é desconhecida:

$$
m_{H}=\sqrt{-2 \mu^{2}}=\sqrt{2} \lambda^{\frac{1}{2}} v
$$

A baixas energias pode-se relacionar $g$ e $M_{W}$ com a constante de Fermi, usando para isso, por exemplo, o elemento de matriz para o espalhamento $e \nu_{\mu} \rightarrow \nu_{e} \mu:$

$$
\frac{G_{F}}{\sqrt{2}}=\frac{g^{2}}{8 M_{W}^{2}}
$$

Com isso obtém-se o valor de $v$ :

$$
v=\frac{2 M_{W}}{g}=246 \mathrm{GeV}
$$

Pode-se definir o parâmetro $\rho$ como:

$$
\rho=\frac{\frac{g_{Z}^{2}}{M_{Z}^{2}}}{\frac{g^{2}}{M_{W}^{2}}}=\frac{M_{W}^{2}}{M_{Z}^{2} \cos \theta_{W}^{2}}=1
$$

Esse parâmetro é, na realidade, a razão entre os acoplamentos da corrente neutra e da corrente carregada na teoria de interação efetiva de quatro 
fermions descrita por Fermi, e constitui um vínculo importante para as extensões do Modelo Padrão.

Conforme foi apresentado acima, a geração dá massa dos bosons de gauge decorre naturalmente da adição do escalar $\Phi$ na Lagrangiana com o $v$ (valor esperado do vácuo) diferente de zero, ou seja, da quebra espontânea de simetria (compare com as eq. (2.18) e (2.19)). No entanto, para gerar massas para os fermions, é preciso adicionar à Lagrangiana termos de Yukawa do tipo:

$$
\begin{aligned}
\mathcal{L}_{f}= & \sum_{i=1}^{3} \sum_{j=1}^{3} \tilde{G}_{i j} \bar{f}_{1 i R}\left(\tilde{\Phi}^{\dagger} F_{j L}\right)+\tilde{G}_{i j}^{\dagger}\left(\bar{F}_{j L} \tilde{\Phi}\right) f_{1 i R} \\
& +G_{i j} \bar{f}_{2 i R}\left(\Phi^{\dagger} F_{j L}\right)+G_{i j}^{\dagger}\left(\bar{F}_{j L} \Phi\right) f_{2 i R}
\end{aligned}
$$

onde $i=1,2,3$ indica a que geração pertence o fermion (quark ou lepton). $G_{i j}$ e $\tilde{G}_{i j}$ são constantes de acoplamento complexas. Aqui estão sendo usados os auto-estados de interação.

Os quarks e leptons podem ser tratados separadamente, pois não se misturam, ou seja, um quark nunca se transforma num lepton e vice-versa (conservação do número leptônico). Portanto, é possível escrever uma Lagrangiana $\mathcal{L}_{f}$ para os quarks $\left(\mathcal{L}_{q}\right)$ e outra para os leptons $\left(\mathcal{L}_{l}\right)$.

Além disso, o tratamento para leptons e quarks difere em um ponto essencial, pois embora os quarks apresentem mistura de gerações, com os leptons isso não ocorre.

Para os quarks, $F_{i L}=\left(\begin{array}{c}u_{i} \\ d_{i}\end{array}\right)_{L}, f_{1 i R}=u_{i R}$ e $f_{2 i R}=d_{i R}$.

No espaço de gerações as matrizes de massa dos quarks são dadas por:

$$
\begin{aligned}
\mathcal{A}_{i j}^{u} & =\frac{v}{\sqrt{2}} \tilde{G}_{i j} \\
\mathcal{A}_{i j}^{d} & =\frac{v}{\sqrt{2}} G_{i j}
\end{aligned}
$$

Por uma transformação unitária atuando sobre os estados dos quarks que ainda não passaram pela quebra espontânea de simetria, passa-se $\mathcal{A}^{u}$ e $\mathcal{A}^{d}$ para a forma diagonal, cujos auto-estados são os quarks $u, c$ e $t$ para a matriz 
$\mathcal{A}^{u}$ e $d, s$ e $b$ para a matriz $\mathcal{A}^{d}$, de forma que os quarks $u, c$ e $t$ são combinações lineares dos $u_{1}, u_{2}$ e $u_{3}$ e da mesma maneira $d, s$ e $b$ são combinações lineares dos $d_{1}, d_{2}$ e $d_{3}$.

Após a passagem para o gauge unitário, a massa dos quarks fica dada por $m_{q}=k_{q} v$, onde $k$ é o acoplamento do quark com o higgs. As correntes carregadas misturam gerações, embora as neutras não o façam. Por convenção, supõe-se que essa mistura ocorra somente para os estados com $T_{3}=-\frac{1}{2}$, de forma que são obtidos como auto-estados os dubletos $\left(\begin{array}{c}u \\ d^{\prime}\end{array}\right)_{L}$, $\left(\begin{array}{c}c \\ s^{\prime}\end{array}\right)_{L}$ e $\left(\begin{array}{c}t \\ b^{\prime}\end{array}\right)_{L}$ e os singletos $u_{R}, c_{R}, t_{R}$ e $d_{R}, s_{R}, b_{R}$, onde $d_{L}^{\prime}, s_{L}^{\prime}$ e $b_{L}^{\prime}$ representam uma mistura entre os quarks $d, s$ e $b$, que são os auto-estados de massa, cujos coeficientes de mistura são dados pela matriz de CabibboKobayashi-Maskawa. Essa matriz pode ser expressa por quatro parâmetros independentes, três ângulos e uma fase [8].

Não há como deduzir a mistura de quarks (assim como as suas massas), a partir de primeiros princípios no Modelo Padrão.

Para os leptons: $F_{i L}=l_{i L}=\left(\begin{array}{c}\nu_{l i} \\ l_{i}\end{array}\right)_{L}, f_{1 i R}=\nu_{l i R}$ e $f_{2 i R}=l_{i R}, i=e, \mu, \tau$

$$
\mathcal{L}_{l}=\tilde{G}_{l} \overline{\nu_{l}}\left(\tilde{\Phi} \dagger l_{L}\right)+\tilde{G}_{l} \dagger\left(\bar{l}_{L} \tilde{\Phi}\right) \nu_{l R}+G_{l} \bar{l}_{R}\left(\Phi \dagger l_{L}\right)+G_{l} \dagger\left(\bar{l}_{L} \Phi\right) l_{R}
$$

Por não existirem neutrinos de mão direita, conclui-se que:

$$
\tilde{G}_{l}=\tilde{G}_{l} \dagger=0
$$

Substituindo a eq. (2.32) na eq. (2.31) (no gauge unitário):

$$
\mathcal{L}=-\frac{G_{l} v}{\sqrt{2}} \bar{l} l-\frac{G_{l}}{\sqrt{2}} h \bar{l} l
$$

Conclui-se que a massa dos leptons é dada por $\frac{G_{t} v}{\sqrt{2}}$. Os neutrinos não se acoplam com o higgs, e portanto não adquirem massa. Isso decorre da exclusão dos neutrinos de mão direita da Lagrangiana.

Aqui há ainda uma observação a ser feita. No caso do Modelo Padrão, o higgs é introduzido como um dubleto, e há acoplamentos dele com os fermions de mão direita e mão esquerda, como pode ser visto na eq. (2.31). Naturalmente, esse acoplamento tem que ser invariante sob $S U(2)_{L}$, mas não 
é possível construir um acoplamento de três dubletos invariante sob essa simetria. De forma que mesmo que năo houvesse a restrição da não conservação da paridade nas interações fracas (como já foi observado anteriormente), seria preciso que uma das quiralidades dos fermions fosse invariante sob $S U(2)_{L}$. Escolhe-se então a componente de mão direita para ser um singleto invariante sob essa simetria.

\subsection{A Invariância de Gauge}

A essa altura pode-se perguntar por que não colocar os termos de massa na Lagrangiana e ignorar o fato de que ela deixe de ser invariante de gauge. A invariância de gauge é realmente um quesito fundamental? Essa questão foi investigada de maneira bastante original nas ref. [12] e [13].

Quando são considerados gráficos como o da fig. (2.2) em que os dois W's são transversais, a amplitude tende a uma constante quando a energia $E \rightarrow \infty$. Porém, quando os W's são longitudinais, o vetor de polarização $\epsilon_{L}$, dado por $\epsilon_{L}=\left(\frac{|\vec{k}|}{M_{W}}, 0,0, \frac{k_{0}}{M_{W}}\right)$, e que para grandes momentos $|\vec{k}|$ comportase como $\epsilon_{L} \sim \frac{k_{\mu}}{M_{W}}$ introduz em cada vértice um fator $\frac{k^{2}}{M_{W}^{2}}$ e a amplitude fica proporcional a $\frac{g^{2} E^{2}}{M_{W}^{2}}$, comportamento este que viola a unitariedade para grandes energias.

Conclui-se portanto que com somente esse diagrama a teoria não tem bom comportamento a altas energias, e portanto não é renormalizável, inegavelmente um defeito gravíssimo para uma teoria.

As fig. (2.2) e (2.3) apresentam o conjunto completo de diagramas $O\left(g^{2}\right)$ com exceção das contribuições do setor de quebra de simetria. Há cancelamento dos termos de ordem $O\left(g^{2} E^{2} / M_{W}^{2}\right)$ provenientes desses gráficos somente se os acoplamentos $f \bar{f} W$ e $W W W$ tiverem exatamente a forma exigida pela invariância de gauge. Resta ainda um termo de ordem $g E m_{f} / M_{W}$.

$\mathrm{O}$ mesmo ocorre para os diagramas da fig. (2.4). As contribuições dos termos $O\left(g^{2}\right)$ cancelam-se entre si somente se o conjunto completo de gráficos for considerado e os vértices tríplices e quádruplos de bosons vetoriais forem invariantes de gauge, restando nesse caso somente um termo de ordem $g^{2} E^{2} / M_{W}^{2}$ na amplitude. 


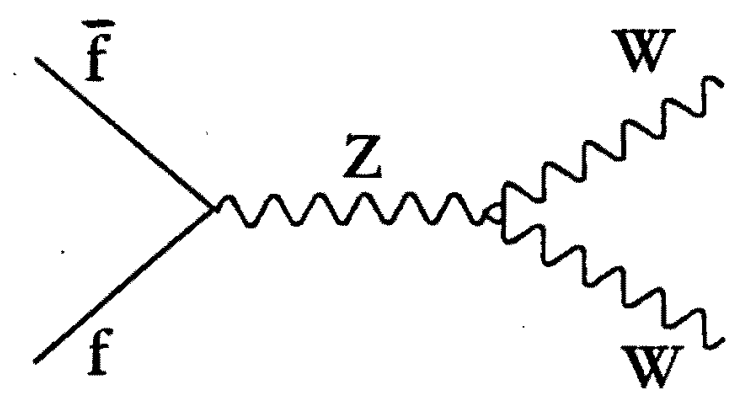

Figura 2.2: Diagrama para o processo $f \vec{f} \rightarrow W W$
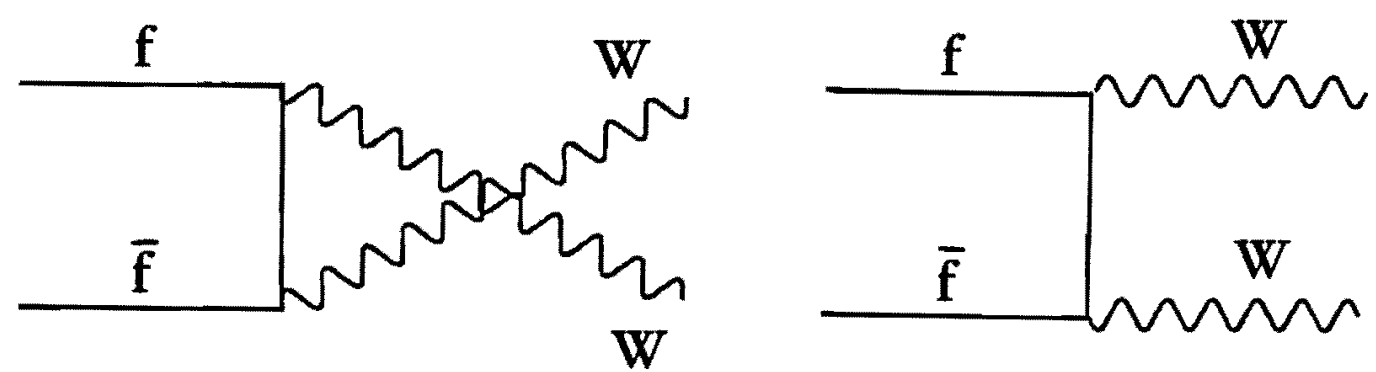

Figura 2.3: Estes gráficos formam, junto com o da figura anterior, o conjunto completo dos diagramas de ordem $g^{2}$. W representa qualquer boson vetorial comm massa. 

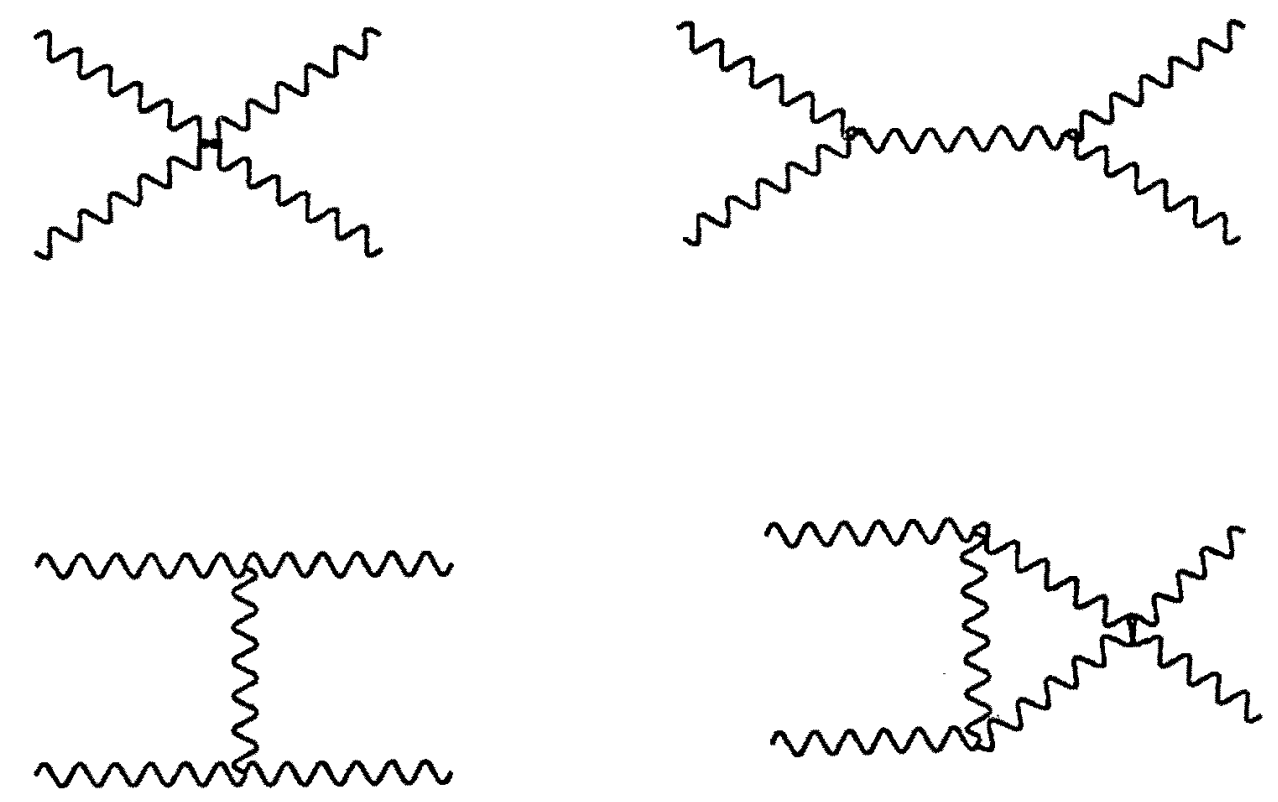

Figura 2.4: Conjunto de diagramas de ordem $g^{2}$, para a reação $W W \rightarrow W W$, onde $W$ é qualquer boson vetorial massivo. Todas as partículas representadas são bosons vetoriais com massa. 
Para que a teoria tenha bom comportamento a altas energias e portanto possa ser usada a teoria de perturbações, é preciso cancelar os dois termos que restarn (um da interação com fermions e outro da interação entre os próprios $W^{\prime} s$ ), e isso vai ocorrer somente com a introdução de alguma nova partícula, ou seja, algum novo boson.

No caso do modelo padrão esse boson tem que ser um escalar, como foi mencionado anteriormente, pois o dubleto introduzido na Lagrangiana (eq. (2.14)) tem quatro componentes (cada uma das componentes do dubleto tem uma componente real e outra imaginária). Quando há a quebra de simetria, para cada gerador do grupo original $(S U(2) \otimes U(1))$ que manteve o vácuo invariante, havia uma componente $\xi_{i}$ (que fazia parte do higgs antes de ser feita a transformação para o gauge unitário), e que se transformou num boson de Goldstone, ou, pelo fato da teoria ser de gauge, na componente longitudinal dos bosons $W^{+}, W_{-}$e $Z_{0}$. Portanto resta somente um grau de liberdade que permite a existência de um escalar.

Os novos gráficos que passam a ser considerados com a interação desse novo boson são os apresentados nas fig. (2.5) e (2.6). O acoplamento $h W W^{\prime}$ tem a forma $\kappa \epsilon \epsilon^{\prime}$, onde a constante de acoplamento tem dimensão de massa e o acoplamento $f \bar{f} h$ tem a forma $\lambda f \bar{f} h$, com $\lambda$ adimensional. Se tomarmos $\kappa \sim g M_{W}$ e $\lambda \sim \frac{g m}{M_{W}}$, todos os termos mal comportados dos gráficos anteriores se cancelam.

Conclui-se que, para que bosons vetoriais com massa tenham bom comportamento em teoria de perturbação, é preciso que:

- a teoria seja de gauge;

- se a teoria for não abeliana, deve existir ao menos um boson escalar $h$ cujos acoplamentos com bosons vetoriais seja proporcional a $g M_{W} \mathrm{e}$ com fermions seja proporcional a $\frac{g m_{f}}{M_{W}}$. Esse boson escalar vai funcionar como um cut off para a teoria. Caso isso não ocorra, tem que haver física nova para os bosons longitudinais em torno de $1 \mathrm{TeV}$, como será visto adiante.

Essas exigências são plenamente respeitadas pela quebra espontânea de simetria conforme apresentada anteriormente, e não resolveria nada, pura e simplesmente colocar a mão os termos de massa na Lagrangiana, relaxando assim a condição de invariância de gauge. 

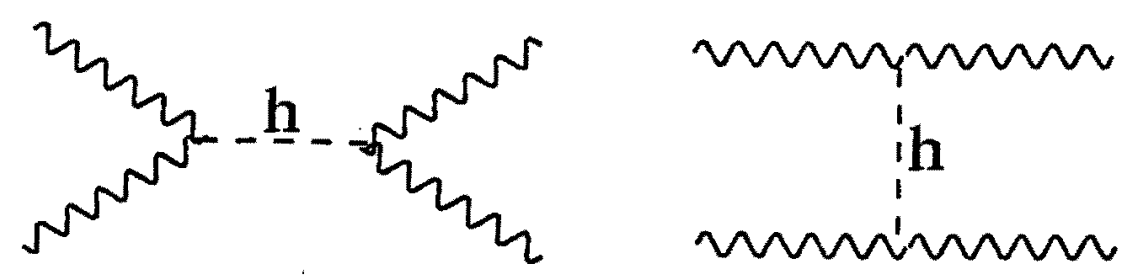

Figura 2.5: Diagramas a nivel de árvore do processo $W W \rightarrow H \rightarrow W W$.

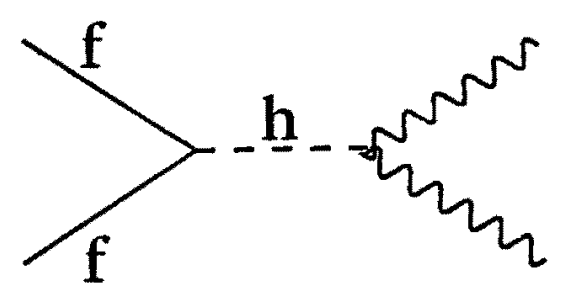

Figura 2.6: Diagrama para a reação $f f \rightarrow H \rightarrow W W$. 


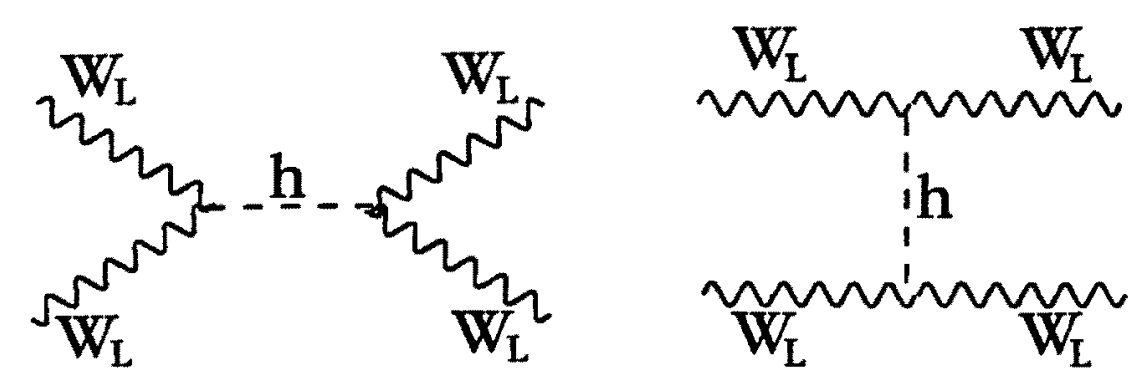

Figura 2.7: Diagramas em que a reação $W_{L}^{+} W_{L}^{-} \rightarrow W_{L}^{+} W_{L}^{-}$ocorre através da troca de um higgs, pelo canal $s$ ou pelo canal $t$.

\subsection{Unitariedade}

Neste ponto do desenvolvimento da teoria, porém, surge outro ponto a ser analisado. Aceitos os fatos de que o higgs é necessário para a renormalizabilidade da teoria, e que ele deve ser um escalar, e com acoplamentos invariantes de gauge com as demais partículas que possuem massa, nenhuma outra restrição precisa ser aplicada? O modelo padrão tal como foi apresentado acima, vai funcionar sempre?

A amplitude dos gráficos de Feynmann mostrados na fig. (2.7), onde é mostrada a reação $W_{L}^{+} W_{L}^{-} \rightarrow W_{L}^{+} W_{L}^{-}$ocorrendo através de trocas de higgs pelos canais $s$ e $t$, quando $s, m_{h}^{2} \gg m_{W^{r}}^{2}, m_{Z}^{2}$ é mostrada a seguir:

$$
\mathcal{A}\left(W_{L}^{+} W_{L}^{-} \rightarrow W_{L}^{+} W_{L}^{-}\right)=-\sqrt{2} G_{F} m_{h_{0}}^{2}\left[\frac{s}{s-m_{h_{0}}^{2}}+\frac{i t}{s-m_{h_{0}}^{2}}\right]
$$

Para $m_{h} \rightarrow \infty$, essa amplitude cresce linearmente com $s$, de forma que ocorre violação da unitariedade. Para $m_{h}$ finito, a onda $J=0$ de uma expansão em ondas parciais para a reação analisada na eq. (2.34) é dada por: 


$$
\begin{aligned}
a_{0} & =\frac{1}{16 \pi s} \int_{-s}^{0} \mathcal{A}\left(W_{L}^{+} W_{L}^{-} \rightarrow W_{L}^{+} W_{L}^{-}\right) d t \\
& =-\frac{G_{F} m_{h}^{2}}{8 \pi \sqrt{2}}\left[2+\frac{m_{h}^{2}}{s-m_{h}^{2}}-\frac{m_{h}^{2}}{s} \ln \left(1+\frac{s}{m_{h}^{2}}\right)\right] .
\end{aligned}
$$

Portanto, para $s \gg m_{h}^{2}$,

$$
a_{0}=-\frac{G_{F} m_{h}^{2}}{4 \pi \sqrt{2}}
$$

Como a unitariedade da amplitude da onda parcial exige $a_{J} \leq 1$, chega-se a um limite para a massa do higgs da ordem de $750 \mathrm{GeV}$. Na ref. [14] esse cálculo é feito levando-se em consideração as amplitudes das ondas parciais com $J=0$ para o conjunto completo de canais acoplados envolvendo $W, Z$ e $h$ para os estados $W^{+} W^{-}, \sqrt{1 / 2} Z_{L} Z_{L}, Z_{L} h$ e $\sqrt{1 / 2} h h$, do qual se obtém um limite ainda mais restritivo dado por $m_{h}^{2} \leq \frac{4 \pi \sqrt{2}}{3 G_{F}} \simeq(700 \mathrm{GeV})^{2}$. Acima deste limite, a amplitude a nível de árvore viola a unitariedade, se a energia no centro de massa $s$ for suficientemente grande.

Para que a unitariedade da matriz de espalhamento $S$ seja restabelecida, é preciso que exista nova física, que se manifestaria através da formação de ressonâncias, como por exemplo mesons vetoriais tipo $\rho$, em espalhamentos $W W[15]$.

Em 1971 't Hooft [9] mostrou que existe um gauge no qual as regras de Feynman levam a um número finito de divergências ultravioletas. Em seguida Lee e Zinn-Justin [10] e 't Hooft e Veltman [11] mostraram que essas divergências podem ser reabsorvidas através de uma redefinição dos parâmetros da teoria, ou seja, que a teoria é renormalizável.

\subsection{Problemas com o Modelo Padrão}

Até o momento não há resultado experimental que contradiga o Modelo Padrão. Pelo contrário, dados tomados no CERN e no FERMILAB mostram concordância sempre crescente com ele. Não obstante, há todo um setor do modelo, o de quebra de simetria, que não foi testado. Não foram observados experimentalmente, de forma direta, nem o higgs e nem o $\nu_{\tau}$. 
É necessário obter várias informações a respeito da matriz de CabibboKobayashi-Maskawa, bem como das massas dos neutrinos. Além disso ele apresenta uma série de problemas, que não deveriam ser esperados de uma teoria final.

Um deles, por exemplo, é a grande quantidade de parâmetros livres que ele apresenta. Pelo fato da teoria ser invariante por uma simetria de gauge local, esse setor (o de gauge), é extremamente bem definido, com seus acoplamentos, massas dos bosons vetoriais, etc. determinados teoricamente. Enquanto a parte fermiônica da teoria fica bastante indefinida, tanto em termos de quantidade (número de gerações - há determinação experimental de que existem três gerações ${ }^{1}$, mas não há como derivar isso da teoria) quanto em termos de propriedades de transformações. Somente suas propriedades relacionadas com os bosons de gauge são bem conhecidas. As informções sobre as massas dos fermions precisam ser obtidas experimentalmente, sendo todas elas parâmetros da teoria (6 para os quarks, e 6 para os leptons), além de 4 parâmetros que definem a matriz de mistura dos quarks, a matriz de Cabibbo-Kobayashi-Maskawa (isso supondo-se que realmente não haja mistura entre os neutrinos, ou seja que a massa dos neutrinos seja nula); o ângulo de mistura eletrofraca, a carga do elétron, etc.

O setor escalar é menos determindo ainda, tanto que há um número muito grande de extensões do Modelo Padrão, e são extremamente genéricas as restrições impostas a ele; o único grande vínculo teórico para esse setor é a invariância de gauge local. Considerando-se o mecanismo proposto pelo Modelo Padrão, surge como parâmetro adicional a massa do higgs.

Nem a própria idéia da unificação das forças é fundamental na teoria. As forças elétrica e fraca são unificadas através da definição da hipercarga, mas continuam existindo dois acoplamentos diferentes, $g$ e $g^{\prime}$, e é experimentalmente que se verifica o fato de que eles têm a mesma ordem de grandeza, levando $\theta_{W}$ a ter um valor que não seja próximo nem de 0 nem de $\frac{\pi}{2}$, de forma que há uma quantidade considerável de mistura que faz as forças parecerem conectadas. Essa unificação é introduzida à mão na teoria.

Por outro lado, é inegável que a idéia de unificação é atraente, principalmente quando se trata das forças forte, fraca e elétrica, pois as três são bem representadas por teorias de gauge, que diferem apenas pelo fato

\footnotetext{
${ }^{1} \mathrm{O}$ número de neutrinos leves, com acoplamentos dados pelo Modelo Padrão é $N_{\nu}=$ $2.983 \pm 0.025[22]$
} 

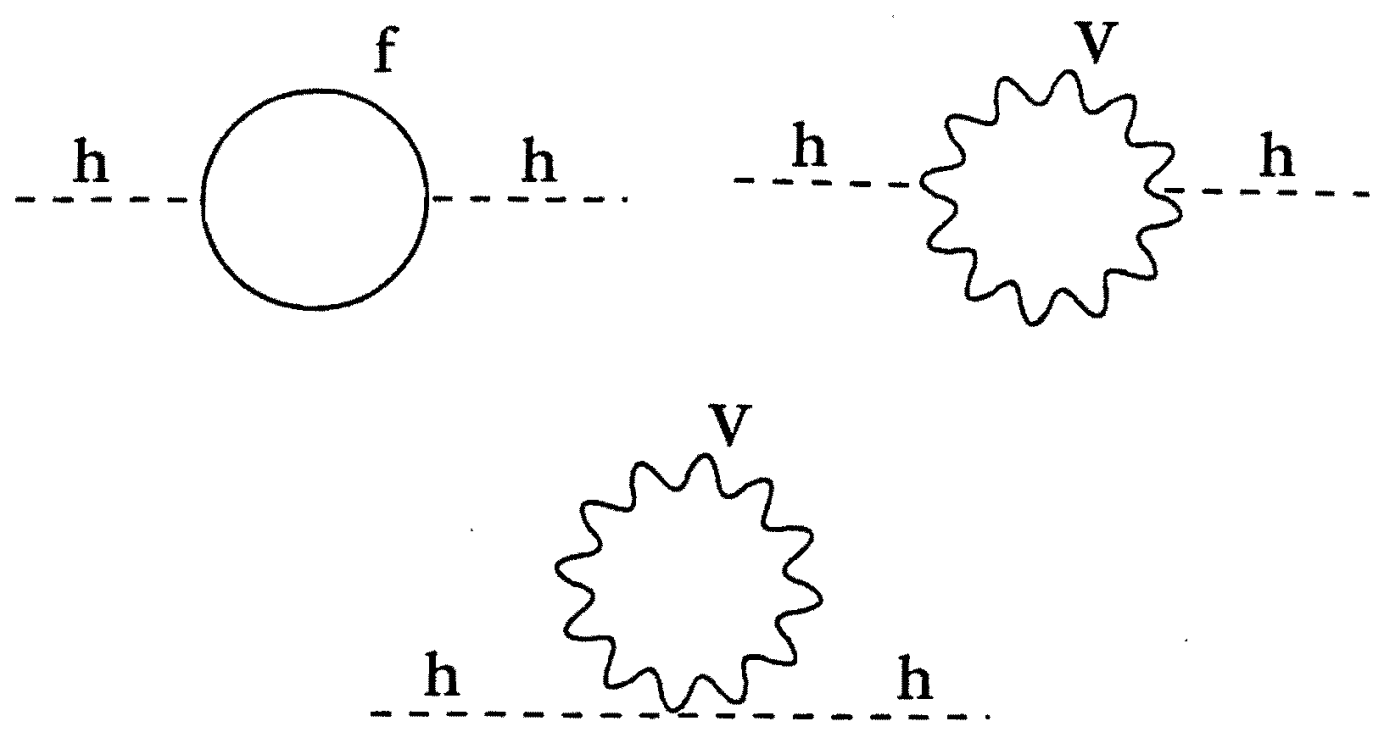

Figura 2.8: Diagramas que contribuem a nivel de um loop para a correção na massa do higgs.

do seu estado de vácuo respeitar ou não a invariância de gauge. Supondose que seja possível essa unificação, o menor grupo possivel que engloba $S U(3)_{C O R} \otimes S U(2)_{L} \otimes U(1)_{Y}$ seria $S U(5)$. A quebra

$$
S U(5) \longrightarrow S U(3)_{C O R} \otimes S U(2)_{L} \otimes U(1)_{Y}
$$

ocorreria numa escala $\Lambda^{2} \sim 10^{14} \mathrm{GeV}$.

Mas nesse ponto, surge outro problema difícil de lidar para o Modelo Padrão: a questão da hierarquia de gauge, ou naturalidade. Já foi mencionada qual é expressão para a massa do higgs no nível de árvore (eq. (2.23)), porém seu valor muda muito quando são levadas em conta as correções radiativas devidas a loops de bosons (vetoriais e escalares) e fermions que são mostradas na fig.2.8. As integrais de loop divergem quadraticamente. Se o Modelo Padrão não é uma teoria final, teria que valer até a escala de quebra de simetria da nova física, $\Lambda^{2}$, de forma que seja possivel calcular $m_{h}$ numa escala $p^{2}$ ajustando $m_{0_{h}}$ como sendo esse valor na escala $\Lambda^{2}$. Então: 


$$
m_{h}^{2}\left(p^{2}\right)=m_{0_{h}}^{2}\left(\Lambda^{2}\right)+C g^{2} \int_{p^{2}}^{\Lambda^{2}} d k^{2}+\ldots
$$

O parâmetro $m_{h_{0}}$ deve ser da ordem de $\Lambda$ para que $m_{h} \sim 10^{2}$ ou $10^{3}$ GeV. Por exemplo, se $m_{h}=1 \mathrm{TeV}$ e $\Lambda=M_{\text {Planck }}$, o cancelamento do lado direito da equação tem que ser da ordem de 16 casas decimais. A teoria da renormalização permite fazer correções de qualquer ordem sem perguntas do tipo de onde veio esse termo, o que o torna necessário, etc., supondo sempre que deve haver uma teoria mais ampla que a que está sendo utilizada, dentro da qual há um bom motivo para tal cancelamento. Mas mesmo assim, uma correção exigindo tamanha precisão soa pouco natural. Esse problema é característico das teorias quadraticamente divergentes, e as únicas teorias em $3+1$ dimensões que são renormalizáveis, e apresentam esse tipo de divergência são as teorias envolvendo escalares elementares. É o chamado problema de naturalidade. 


\section{Capítulo 3}

\section{Higgs}

Neste capítulo serão apresentados os mais importantes modos de produção e decaimento do higgs. Essa análise depende da massa da partícula, portanto, será feita antes uma breve descrição dos possíveis vínculos a serem impostos a esse parâmetro.

\subsection{Determinação da Massa}

A massa do higgs é um dos parâmetros livres do modelo padrão, e como era de se esperar, esse fato influi nos seus modos de produção e decaimento, tornando mais difícil o trabalho para sua deteção. Por esse motivo, há vários anos vem se tentando determinar limites teóricos para $m_{h}$, obtidos de fontes tão diferentes quanto correções radiativas, um limite a partir do qual a teoria torna-se trivial (limite superior), e o limite de estabilidade do vácuo (inferior). Além desses, há limites experimentais (inferiores) que já foram obtidos.

\subsubsection{Correções Radiativas}

Há três parâmetros definidos de forma cuidadosa, que exercem papel importante na teoria eletrofraca [18]. São eles:

1. constante de estrutura fina

$$
\alpha=1 / 137.03599, \text { conhecida com a precisão } \Delta \alpha=0.045 \mathrm{ppm} \text {. }
$$


2. constante de decaimento do muon

$G_{F}$, determinada, por definição a partir da meia vida do muon, pela relação:

$$
\frac{1}{t_{\mu}}=\frac{G_{F}^{2} m_{\mu}^{5}}{192 \pi^{3}} f\left(\frac{m_{e}^{2}}{m_{\mu}^{2}}\right)\left[1+\frac{3}{5} \frac{m_{e}^{2}}{m_{\mu}^{2}}\right]\left[1+\frac{\alpha}{2 \pi}\left(\frac{25}{4}-\pi^{2}\right)\left(1+\frac{2 \alpha}{3 \pi} \ln \left(\frac{m_{\mu}}{m_{e}}\right)\right)\right],
$$

onde $f(x)=1-8 x-12 x^{2} \ln x+8 x^{3}-x^{4}$.

$$
G_{F}=1.16639(1) \times 10^{-5} \mathrm{GeV}^{-2}\left(\Delta G_{F}=8.5 \mathrm{ppm}\right)
$$

As correções radiativas na eq. (3.1) são completamente conhecidas ao nível de um loop.

3. $m a s s a d o Z^{0}$

$m_{Z}=91.1895 \pm 0.0044 \mathrm{GeV},\left(\Delta m_{Z}=48 \mathrm{ppm}\right)$, sendo $m_{Z}$ calculado como a posição do polo no propagador do $Z^{1}$.

As correções radiativas associadas à evolução de $\alpha$ têm um papel importante na física eletrofraca. $\mathrm{O}$ valor dessa constante $\alpha(M)$ numa determinada escala de massa $M$ é dado por:

$$
\alpha\left(m_{Z}\right)=\frac{\alpha}{1-\Delta \alpha} .
$$

sendo que $\Delta \alpha$ depende do esquema de renormalizaçăo usado.

A partir dos três parâmetros apresentados acima, são calculados, entre outros parâmetros, $\sin ^{2} \theta_{W}$ e $m_{W}$. Ao nível de árvore, essas expressões são dadas pelas eq. (2.8), (2.19) e $(2.24)^{2}$.

\footnotetext{
${ }^{1}$ Há algumas sutilezas a serem consideradas na definição dessa massa, mas não serão levadas em conta aqui. Há uma pequena discussão sobre o assunto na ref. [18]

${ }^{2}$ Essa última relação deixa de valer em modelos onde sejam introduzidos tripletos de higgs ou outras representações que não apenas dubletos e singletos de escalares, e nesses casos é necessário introduzir uma definição mais geral. Aqui está sendo considerado somente o modelo padrão.
} 
Porém, para se aproximar dos valores reais dessas grandezas, é preciso considerar ordens superiores em teoria de perturbação, são necessárias definições precisas dos parâmetros envolvidos. $m_{W}$ é definido como o polo no propagador do $W$, de forma análoga a $m_{Z}$. Definindo-se $\sin ^{2} \theta_{W}=1-\frac{m_{W}^{2}}{m_{Z}^{2}}$, chega-se a:

$$
\sin ^{2} \theta_{W} \cos ^{2} \theta_{W}=\frac{\pi \alpha /\left(\sqrt{2} G_{F}\right)}{m_{Z}^{2}(1-\Delta r)}
$$

onde $\Delta r$ é a correção radiativa. Há outras formas possíveis de se definir essas grandezas, mas a conclusão geral que será apresentada a seguir é a mesma em todos os casos.

O valor de $\Delta r$ é pois, crucial na determinação precisa do ângulo de Weinberg e da massa do $W$. A contribuições dominantes para esse cálculo provém de loops fermiônicos e são do tipo $\Delta r \sim-\frac{m_{f}^{2}}{m_{Z}^{2}}$. Sem dúvida a maior contribuição vem do termo $f=t$ (quark top).

Há também correções devidas a loops de bosons eletrofracos, $W^{ \pm}, Z^{0}$, $\gamma$ e $h$. Essas correções afetam auto-energias, vértices e diagramas de caixa, porém são muito menores que as correções fermiônicas. O comportamento dominante, devido ao higgs, para grandes valores de $m_{h}$ é proporcional a $\ln \left(\frac{m_{h}^{2}}{m_{Z}^{2}}\right)$.

É importante frisar que as duas contribuições têm sinais opostos, e também que a contribuição devida a $m_{t}$ é quadrática, enquanto a devida ao higgs é logaritmica. Por esse motivo, a comparação do valor experimental de $\Delta r$ com o valor esperado teoricamente, permitiu a antecipação do valor da massa do quark top com razoável correção. O mesmo não é possível para o higgs, pois as correções devidas a ele são pequenas, e esse valor fica perdido nos erros experimentais e nas incertezas ainda existentes no valor das massas dos quarks ${ }^{3}$. M. Veltman [19] foi o primeiro a mostrar essa dificuldade, chamando-a de screening.

\footnotetext{
${ }^{3}$ Para uma massa do higgs da ordem de $3000 \mathrm{GeV}$, a correção será da ordem de $0.4 \%$.
} 


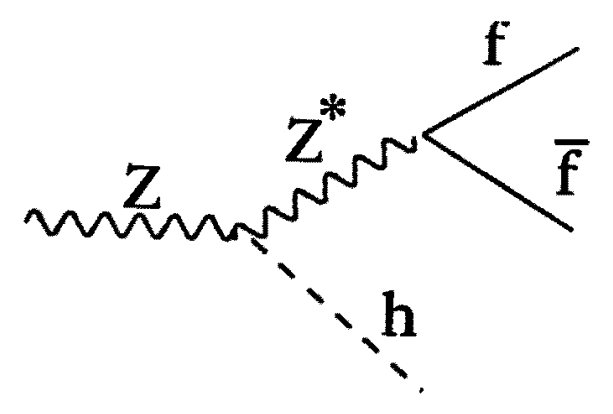

Figura 3.1: Diagrama para o processo $Z \rightarrow Z h \rightarrow l^{+} l^{-} h$.

\subsubsection{Limites Experimentais}

A análise dos eventos do LEP permite garantir, com 95\% C.L., que não existe um higgs com massa inferior a $58.4 \mathrm{GeV}$ [22]. Para a obtenção desse limite foram analisados dados das experiências DELPHI, OPAL ,L3 e ALEPH.

Este resultado foi obtido a partir de análise de dados obtido com o processo cujo diagrama é apresentado na fig. (3.1), conhecido como processo de Björken no qual o $Z^{0}$ irradia um higgs, e em seguida decai num par de fermions (este modo de produção, assim como o de decaimento serão analisados a adiante ainda neste capítulo).

\subsubsection{Limites Teóricos}

Há um limite teórico superior (trivialidade) e outro inferior (estabilidade do vácuo) para a massa do higgs. Será mostrado a seguir que com o valor atualmente aceito para a massa do top, o limite inferior não exerce influência alguma.

É comum que a exigência de unitariedade da teoria seja apresentada como um limite para a massa do higgs, mas na verdade, ela vai apenas traçar a linha demarcatória até onde poderá ser usada a teoria de perturbação, a partir da qual, o setor de quebra de simetria deixará de ser do tipo eletrofraco, para passar a ser forte. 


\section{Trivialidade - Limite Superior para a Massa}

A equação do grupo de renormalização para a constante de acoplamento $\lambda$, no limite em que as constantes de acoplamento de Yukawa e de gauge podem ser desprezadas, torna-se [23]:

$$
\frac{d \lambda_{R}(t)}{d t}=\frac{3 \lambda_{R}^{2}}{2 \pi^{2}}+\ldots
$$

onde são mostradas contribuiçôes de um loop para a função $\beta$. Assumindo essa contribuição exata, integra-se a eq (3.5) desde $t=0(Q=v)$ até $t=$ $\log \frac{\Lambda}{v}(Q=\Lambda)$, sendo $\lambda_{0}=\lambda_{R}(t=0)$ a constante de acoplamento calculada na escala $v$, e $\lambda(t)=\lambda_{R}(t)$, resultando:

$$
\lambda_{R}(t)=\frac{\lambda_{0}}{1-\frac{3}{2 \pi^{2}} \lambda_{0} t} .
$$

O sinal negativo no denominador (onde ocorre um polo de Landau), típico de teorias não assintoticamente livres, implica no crescimento de $\lambda(t)$ até um valor infinito, obtido para $\frac{3}{2 \pi^{2}} \lambda_{0} t=1$. Para evitar esse polo é necessário impor a condição:

$$
\frac{3}{2 \pi^{2}} \lambda_{0} t=\frac{3}{2 \pi^{2}} \frac{m_{h}^{2} G_{F}}{\sqrt{2}} \ln \frac{\Lambda}{v}<1 .
$$

Essa condição é equivalente a:

$$
m_{h}<\frac{893 \mathrm{GeV}}{\sqrt{\ln \Lambda / v}}
$$

Portanto, $m_{h}<144 \mathrm{GeV}$ para $\Lambda=10^{19}$ (escala de Planck), $m_{h}<$ $165 \mathrm{GeV}$ para $\Lambda=10^{15}$ (escala de Grande Unificação), $m_{h}<675 \mathrm{GeV}$ para $\Lambda=10^{3}$ (escala de quebra de simetria eletrofraca), respectivamente, o que implica que o Modelo Padrão é uma teoria efetiva, que descreve a física abaixo da escala do parâmetro de corte $\Lambda$. O limite imposto a $m_{h}$ é chamado limite de trivialidade.

Embora esse seja um modelo simplificado, esses resultados se mantêm aproximadamente os mesmos quando é incluído um tratamento mais refinado para o grupo de renormalização, e também em resultados obtidos sem teoria 
de perturbações em simulações usando teoria de gauge na rede. Nesse último caso, obtém-se como limite superior $m_{h} \lesssim 750 \mathrm{GeV}$ [24].

Este fenômeno está presente também na QED, todavia ele ocorreria apenas além da escala de massa de Planck, de forma que é possível supor que nessa escala a teoria esteja inserida em outra teoria mais geral.

\section{Estabilidade do Vácuo}

O limite inferior para a massa do higgs, obtido primeiramente por Linde e Weinberg [25], baseia-se na exigência de estabilidade do vácuo, quando são consideradas correções radiativas para o potencial efetivo dado pela eq. (2.13).

Considerando-se as correções radiativas a nível de um loop, o potencial citado acima passa a ser:

$$
V=-\mu^{2} \Phi^{2}+\lambda \Phi^{4}+\beta_{\lambda} \Phi^{4} \ln \frac{\Phi^{2}}{Q_{0}^{2}}
$$

sendo:

$$
\beta_{\lambda}=\frac{1}{16 \pi^{2} v^{4}}\left(3 \sum_{V} m_{V}^{4}+\sum_{S} M_{S}^{4}-12 \sum_{f} m_{f}^{4}\right)
$$

onde a soma em $f$ indica uma soma sobre todos os fermions (sendo que o único que precisa ser considerado é o top, pois os outros possuem massa pequena quando comparada à dos bosons vetoriais) e a soma em $V$ indica uma soma sobre os bosons vetoriais. A soma em $\mathrm{S}$ indica uma soma sobre os bosons escalares da teoria; no Modelo Padrão há somente um higgs, e como se está procurando um limite inferior para sua massa, ela deve ser pequena o suficiente para não precisar ser levada em conta no cálculo. Além disso $Q_{0}$ é uma escala de massa da ordem de $v$.

O mínimo do potencial em $\Phi=\frac{v}{\sqrt{2}}$ fornece o valor de $\mu^{2}$ :

$$
\left.\frac{\partial V}{\partial \Phi}\right|_{\Phi=v / \sqrt{2}}=0
$$

levando a:

$$
\mu^{2}=\lambda v^{2}+v^{2} \beta_{\lambda} \ln \frac{v^{2}}{2 Q_{0}^{2}}+\frac{v^{2}}{2} \beta_{\lambda}
$$


A massa do higgs é dada por:

$$
\begin{aligned}
m_{h}^{2} & =\left.\frac{1}{2} \frac{\partial^{2} V}{\partial \Phi^{2}}\right|_{\Phi=v / \sqrt{2}} \\
& =2 v^{2}\left[\lambda+\beta_{\lambda}\left(\ln \left(\frac{v^{2}}{2 Q_{0}^{2}}\right)+\frac{3}{2}\right)\right]
\end{aligned}
$$

onde foi substituinda a eq.( 3.12$)$.

$$
\begin{aligned}
m_{h}^{2} & =v^{2} \beta_{\lambda}+2 v^{2}\left[\lambda+\beta_{\lambda}\left(\ln \frac{v^{2}}{2 Q_{0}^{2}}+1\right)\right] \\
& =v^{2} \beta_{\lambda}-\frac{8 V\left(\Phi_{0}\right)}{v^{2}}
\end{aligned}
$$

Quando não há quebra de simetria: $V(0)=0$. Para que haja quebra de simetria, é necessário impor:

$$
V(v / \sqrt{2})<V(0) \Rightarrow V(v / \sqrt{2})<0
$$

o que implica em:

$$
m_{h}^{2}>\beta_{\lambda} v^{2}
$$

que leva ao limite inferior:

$$
m_{h}^{2}>\frac{3}{16 \pi^{2} v^{2}}\left[2 m_{W}^{4}+m_{Z}^{4}-4 m_{i}^{4}\right]
$$

onde foram desprezadas as massas dos quarks mais leves que o top. No entanto para o valor da massa do top, que atualmente é bem conhecido, esse limite é negativo, portanto não impondo restrição nenhuma a $m_{h}$.

\subsection{Modos de Decaimento}

A determinação dos modos de decaimento do higgs sem dúvida varia conforme a faixa de massa em que ele se encontra.

Há um Teorema de Baixa Energia para as interações do boson de Higgs [26], que é bastante utilizado na obtenção das larguras de decaimento, e que relaciona as amplitudes de dois processos que diferem pela inserção de um higgs 
com momento nulo. Ele será enunciado aqui, para melhor compreensão de alguns resultados apresentados a seguir [27].

\subsubsection{Teorema de Baixas Energias para Interações do Higgs}

De acordo com o que foi desenvolvido na sec. 2.2 a Lagrangiana de interaçôes do Modelo Padrão pode ser escrita como:

$$
\mathcal{L}_{i n t}=-\left(1+\frac{h}{v}\right) \sum_{f} m_{f} \vec{f} f-\left(1+\frac{h}{v}\right)^{2}\left(m_{W}^{2} W^{\mu+} W_{\mu}^{-}+\frac{1}{2} m_{Z}^{2} Z_{\mu} Z^{\mu}\right)
$$

onde soma-se sobre todos os fermions da teoria. Considerando-se um higgs com quadrimomento nulo $i \partial_{\mu} h=0$, onde $h$ representa o campo do higgs. Isso implica que $h$ é um campo constante. Da eq. (3.20), segue que o efeito de um campo constante $h$ é equivalente a redefinir todos os parâmetros de massa da teoria: $m_{i} \rightarrow m_{i}+\frac{h}{v}$.

Isso implica imediatamente no teorema de baixa energia:

$$
\lim _{p_{h} \rightarrow 0} \mathcal{M}(A \rightarrow B+h)=\frac{1}{v}\left(\sum_{f} m_{f} \frac{\partial}{\partial m_{f}}+\sum_{V} m_{V} \frac{\partial}{\partial m_{V}}\right) \mathcal{M}(A \rightarrow B),
$$

onde a soma sobre $V$ inclui os bosons $W^{ \pm}$e $Z^{0}$.

Há uma demonstração desse teorema na ref. [27], para a reação gluon $\rightarrow$ gluon h $(g \rightarrow g h)$.

\subsubsection{Decaimento em Fermions}

Da eq. 2.33 deduz-se que o acoplamento do higgs com fermions é dado pela Lagrangiana:

$$
\mathcal{L}=-\left(\sqrt{2} G_{F}\right)^{1 / 2} m_{f} h f \bar{f}
$$

o que leva a uma largura parcial, a nível de árvore, dada por: 


$$
\Gamma(h \rightarrow f \bar{f})=c_{f} \frac{G_{F} m_{f}^{2} m_{h}}{4 \pi \sqrt{2}}\left[1-\frac{4 m_{f}^{2}}{m_{h}^{2}}\right]^{3 / 2}
$$

sendo $c_{f}=1$ quando o fermion em questão é um lepton, e $c_{f}=3$ quando é um quark.

Portanto, o acoplamento é proporcional à massa do fermion, e se o higgs for decair em um par de fermions, há uma grande probabilidade de que seja no de maior massa possível. Considerando-se o limite inferior obtido experimentalmente para a massa do higgs (pag. (29)), somente os decaimentos em bottoms e tops precisam ser considerados, pois os outros possuirão uma taxa de ramificação muito pequena.

As correções eletrofracas a esse resultado ([28]) e ([29]) são significativas somente para um higgs pesado (da ordem de $10 \%$ para $m_{h}=1 \mathrm{TeV}$ ). Já as correções devidas à QCD ([30]), ([31]) e ([32]) são consideráveis, sendo que a taxa de decaimento $h \rightarrow b \vec{b}$, por exemplo, é reduzida por um fator de 0.6 ; essas correções são ainda maiores no caso de decaimentos em um par $t \bar{t}$.

Mas o decaimento em top nunca chega a ser muito importante, porque para um higgs com massa suficiente para produzir um par $t \bar{t}$, outros fatores já passaram a influir no processo, e seus decaimentos serão diferentes, como será visto adiante.

Portanto, para um higgs com massa $m_{h}<2 m_{W}$ o decaimento em um par de bottons tem uma razão de ramificação de aproximadamente $90 \%$ [33]. Em seguida aparecem os decaimentos em $c \bar{c}$ e $\tau^{+} \tau^{-}$, com razão de ramificação de aproximadamente 5\% [33]. Porém, esses decaimentos provavelmente não poderão ser vistos [34] num anel de colisão hadrônico, pois a taxa de produção de quark $b$ em reações de QCD nesses anéis, é várias ordens de magnitude maior que a produção de higgs.

Em virtude desse fato, foram procuradas soluçôes alternativas para a deteção do higgs nessa faixa de massa, produzido em colisões hadrônicas. As principais dessas alternativas são $h \rightarrow \gamma \gamma$ e $h \rightarrow Z Z^{*}$.

\subsubsection{Decaimento em Gluons}

Por ser intermediário de uma simetria perfeita, o gluon não se acopla com o higgs, e portanto não adquire massa. Portanto o decaimento $h \rightarrow g g$ ocorre através de um loop de fermions. Como o acoplamento dos fermions com o 


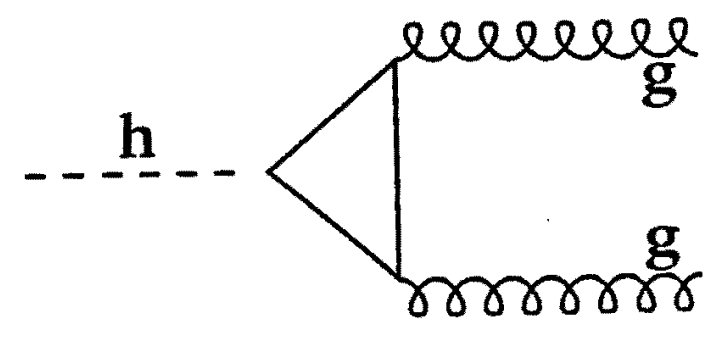

Figura 3.2: Diagrama para o processo $h \rightarrow g g$

higgs é proporcional à massa do fermion, os quarks pesados têm um papel preponderante, o top especialmente. A fig. (3.2) mostra esse processo.

O teorema de baixa energia apresentado na seção (3.2.1) é aplicado para se obter a largura desse decaimento. A fig. (3.3) mostra os dois diagramas envolvidos no cálculo.

A amplitude é dada por:

$$
\mathcal{A}(g \rightarrow g h)=\left(\sqrt{2} G_{F}\right)^{1 / 2} \sum_{q} m_{q} \frac{\partial}{\partial m_{q}} \mathcal{A}(g \rightarrow g)
$$

O diagrama $g \rightarrow g$ é divergente, mas pode ser regularizado por um parâmetro de corte que não afeta o resultado para $g \rightarrow g h$.

A Lagrangiana efetiva para a interação é dada por:

$$
\mathcal{L}=-\left(\sqrt{2} G_{F}\right)^{1 / 2} \frac{\alpha_{s}\left(m_{h}^{2}\right)}{12 \pi} I G_{\mu \nu}^{a} G_{a}^{\mu \nu} h
$$

onde:

$$
\begin{aligned}
& \alpha_{s}\left(m_{h}^{2}\right) \text { é a constante de acoplamento forte calculada em } m_{h}^{2}, \\
& G_{\mu \nu}^{a}=\partial_{\mu} g_{\nu}^{a}-\partial_{\nu} g_{\mu}^{a}, \mathrm{e}
\end{aligned}
$$



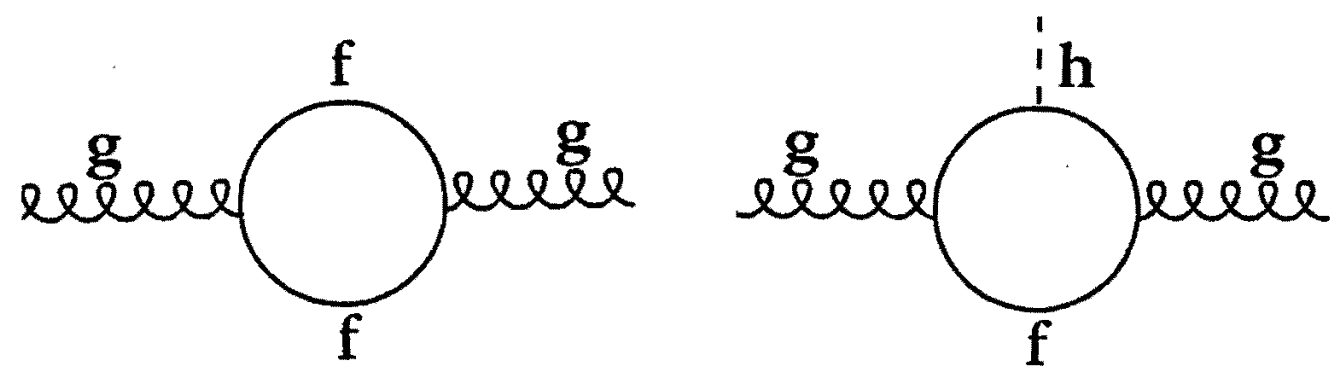

Figura 3.3: Diagrama ilustrando a relação entre o diagrama com um loop de fermions e outro envolvendo $h \rightarrow g g$

$$
I=\sum_{q} I_{q},
$$

$\operatorname{com} I_{q}=\frac{3 \int_{0}^{1} x \int_{0}^{1-x} d y(1-4 x y)}{1-\frac{x y}{\lambda_{q}}-i \epsilon}, \quad$ sendo $\quad \lambda_{q}=\frac{m_{q}^{2}}{m_{h}^{2}}$.

O cálculo da integral acima dá:

$$
I_{q}=3\left[2 \lambda_{q}+\lambda_{q}\left(4 \lambda_{q}-1\right) f\left(\lambda_{q}\right)\right],
$$

onde :

$$
f(\lambda)= \begin{cases}-2\left(\sin ^{-1} \frac{1}{2 \sqrt{\lambda}}\right)^{2} & \lambda>\frac{1}{4} \\ \frac{1}{2}\left(\ln \frac{\eta^{+}}{\eta^{-}}\right)^{2}-\frac{\pi^{2}}{2}-i \pi \ln \frac{\eta^{+}}{\eta^{-}} & \lambda<\frac{1}{4}\end{cases}
$$

sendo $\eta^{ \pm}=\frac{1}{2} \pm \sqrt{\frac{1}{4}-\lambda}$.

Sem dúvida, as duas contribuições mais importantes para $I_{q}$ na eq. (3.26) são $I_{b}$ e $I_{t}$, sendo esse último predominante (para $\lambda_{q} \gg 1, I_{q} \rightarrow 1$ ).

Com essas relações, obtém-se a expressão para a taxa de decaimento: 


$$
\Gamma(h \rightarrow g g)=\frac{G_{F} m_{h}^{3}}{36 \sqrt{2} \pi}\left[\frac{\alpha_{s}\left(m_{h}^{2}\right)}{\pi}\right]^{2}|I|^{2}
$$

Embora seja difícil observar esse decaimento, uma das pretensões das experiências de colisões hadrônicas é determinar bem o acoplamento hgg a partir da produção do higgs pelo processo $g g \rightarrow h$. Esse acoplamento é devido à soma sobre todas as partículas coloridas pesadas que adquiriram massa através do mecanismo de Higgs. Quando comparado com as larguras de decaimentos de $Z \rightarrow h \gamma$ e $h \rightarrow \gamma \gamma$, importantes vínculos sobre possíveis partículas pesadas que participam do loop serão obtidos.

\subsubsection{Decaimento em Fotons}

Esse é um decaimento raro, devendo ocorrer em torno de 3000 eventos por ano no LHC (com energia no centro de massa, $s=14 \mathrm{TeV}$ e luminosidade $\mathcal{L}=10^{34} \mathrm{~cm}^{-2} s$ ), se a massa do higgs for $m_{h} \sim 100 \mathrm{GeV}$. Apesar disso deve ser o melhor canal para se observar um higgs com massa entre 80 e $130 \mathrm{GeV}$.

Formalmente, o cálculo da largura do decaimento $h \rightarrow \gamma \gamma$ é similar ao da reação $h \rightarrow \dot{g g}$, exceto pelo fato de que no primeiro devem ser considerados loops de todas as partículas carregadas (loops fermiônicos com sinal oposto aos de bosons vetoriais) do modelo ${ }^{4}$. Os diagramas que contribuem para essa amplitude são apresentados na fig. (3.4).

A Lagrangiana de interação é [27]:

$$
\mathcal{L}=-\frac{g m_{f}}{2 m_{W}} \bar{\psi} \psi h+g m_{W} W_{\mu}^{+} W^{\mu-} h
$$

O teorema de baixa energia apresentado na sec. (3.2.1) fica:

$$
\mathcal{A}(\gamma \rightarrow h \gamma)=\left(\sqrt{2} G_{F}\right)^{1 / 2}\left(M_{W} \frac{\partial}{\partial M_{W}}+\sum_{f} m_{f} \frac{\partial}{\partial m_{f}}\right) \mathcal{A}(\gamma \rightarrow \gamma)
$$

Assim chega-se à taxa de decaimento:

\footnotetext{
${ }^{4}$ Num modelo com escalares carregados, eles precisariam ser levados em conta também, de forma que a análise desse canal torna-se uma importante fonte de informação sobre a possivel existência de nova física; no entanto a contribuição dos loops escalares é muito menor que a dos fermions e dos bosons vetoriais.
} 


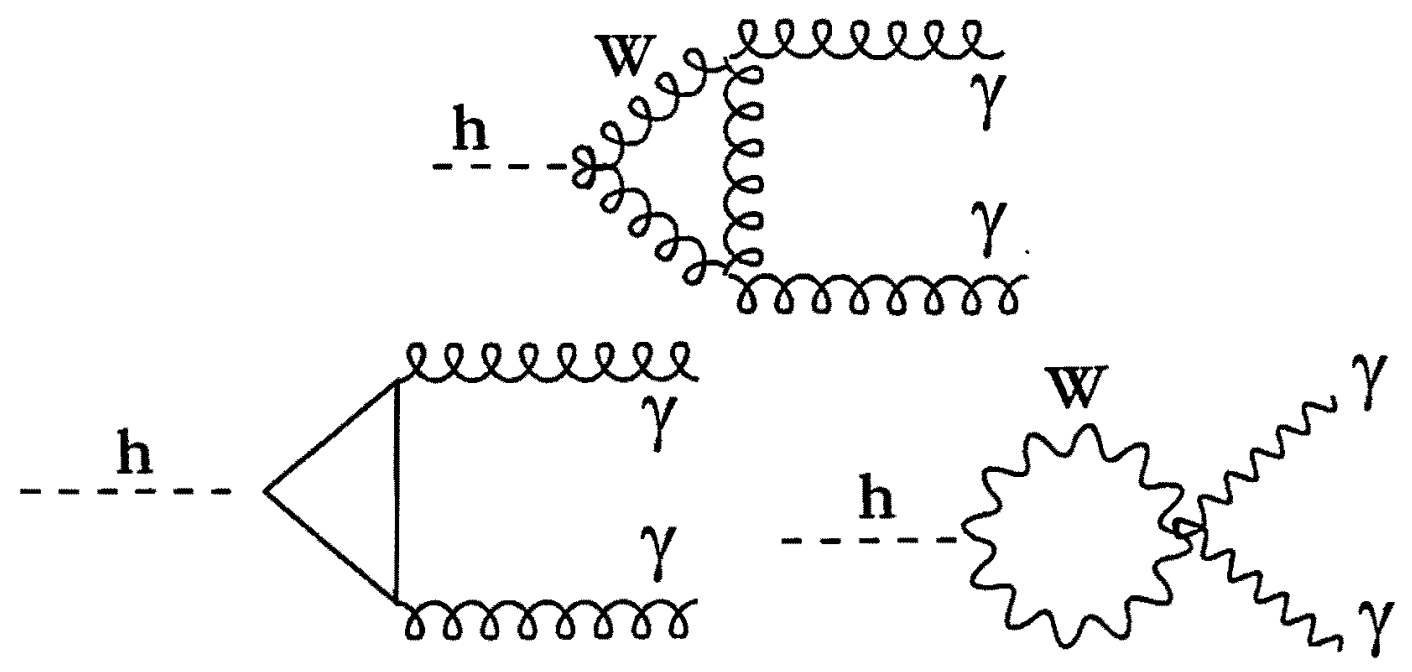

Figura 3.4: Diagramas que contribuem para a reação $h \rightarrow \gamma \gamma$.

$$
\Gamma(h \rightarrow \gamma \gamma)=\frac{G_{F} m_{h}^{3}}{8 \sqrt{2} \pi}\left(\frac{\alpha}{\pi}\right)^{2}|I|^{2}
$$

sendo I dado por:

$$
I=\sum_{q} Q_{q}^{2} I_{q}+\sum_{l} Q_{l}^{2} I_{l}+I_{W}
$$

onde $Q_{f}$ é a carga do fermion. Além disso:

$$
\begin{aligned}
I_{q} & =3\left[2 \lambda_{q}+\lambda_{q}\left(4 \lambda_{q}-1\right) f\left(\lambda_{q}\right)\right] \\
I_{l} & =2 \lambda_{l}+\lambda_{l}\left(4 \lambda_{l}-1\right) f\left(\lambda_{l}\right) \\
I_{W} & =3 \lambda_{W}\left(1-2 \lambda_{W}\right) f\left(\lambda_{W}\right)-3 \lambda_{W}-\frac{1}{2}
\end{aligned}
$$

$\operatorname{com} \lambda_{i}=m_{i}^{2} / m_{h}^{2}$.

A fig. (3.5) apresenta uma comparação entre as contribuições de $W, t$ e $b$ para $h \rightarrow \gamma \gamma$ supondo $m_{t}=150 \mathrm{GeV}$ (o resultado não seria muito diferente para a massa verdadeira do top). As integrais $I_{i}$ são complexas a partir do limite $m_{h}>2 m_{i}$, e tendem a 1 para $m_{h}$ muito abaixo desse limite. 


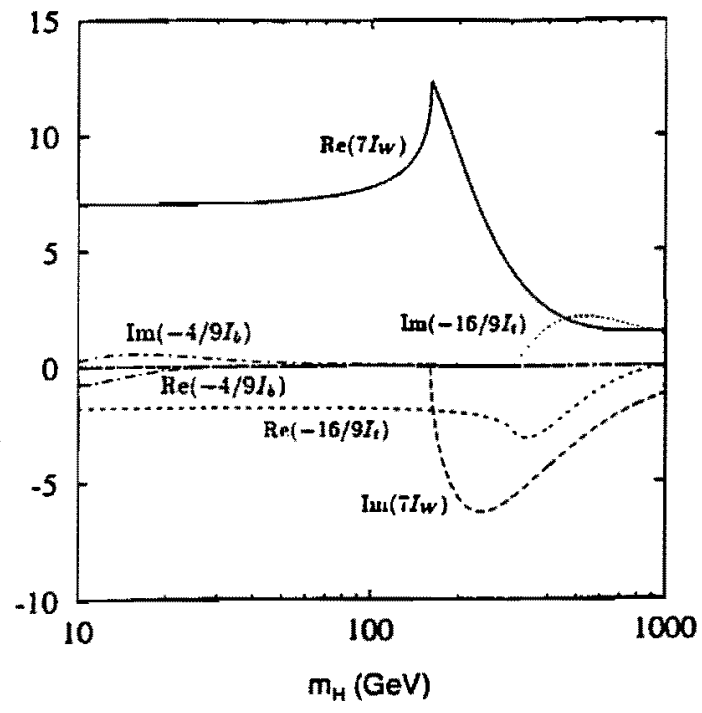

Figura 3.5: Comparação entre as contribuições dos loops de $W, b$ e $t$ para o decaimento $h \rightarrow \gamma \gamma$.

\subsubsection{Decaimento em $Z Z^{*}$ e $W^{*} W$}

Esses modelos de decaimento são especialmente importantes para um higgs cuja massa esteja situada no intervalo $130<m_{h}<180 \mathrm{GeV}$. Nesse caso, um dos bosons produzidos está na camada de massa, e o outro é virtual. As larguras, somadas para todos os canais disponíveis para o $W^{*}$ e o $Z^{*}$ são dadas por [35] [27]:

$$
\Gamma\left(h \rightarrow W^{*} W\right)=\frac{3 g^{4} m_{h}}{512 \pi^{3}} f\left(m_{W} / m_{h}\right)
$$

e

$$
\Gamma\left(h \rightarrow Z^{*} Z\right)=\frac{g^{4} m_{h}}{2048 \pi^{3}} \frac{7-\frac{40}{3} \sin ^{2} \theta_{W}+\frac{160}{9} \sin ^{4} \theta_{W}}{\cos ^{4} \theta_{W}} f\left(\frac{m_{Z}}{m_{h}}\right),
$$

onde

$$
f(x)=-\left|1-x^{2}\right|\left(\frac{47}{2} x^{2}-\frac{13}{2}+\frac{1}{x^{2}}\right)
$$




$$
-3\left(1-6 x^{2}+4 x^{4}\right)|\ln x|+3 \frac{1-8 x^{2}+20 x^{4}}{\sqrt{4 x^{2}-1}} \cos ^{-1}\left(\frac{3 x^{2}-1}{2 x^{3}}\right) .
$$

Esses modos de decaimento são particularmente úteis em anéis de colisão hadrônicos com alta luminosidade, pois podem ser considerados estados finais em que os dois bosons vetoriais produzidos decaiam leptonicamente.

\subsubsection{Decaimento em $Z Z$ ou $W^{+} W^{-}$}

Da eq. (2.17), obtemos a Lagrangiana que relaciona os campos de gauge vetoriais e o higgs:

$$
\mathcal{L}=\left(\sqrt{2} G_{F}\right)^{1 / 2}\left(2 m_{W}^{2} h W_{\mu}^{+} W^{\mu-}+m_{Z}^{2} h Z_{\mu} Z^{\mu}\right)
$$

As taxas de decaimento são dadas por [1]:

$$
\Gamma\left(h \rightarrow W^{+} W^{-}\right)=\frac{G_{F}}{8 \pi \sqrt{2}} m_{h}^{3}\left(1-4 \lambda_{W}\right)^{1 / 2}\left(12 \lambda_{W}^{2}-4 \lambda_{W}+1\right),
$$

onde $\lambda_{W}=\left(\frac{m_{W}}{m_{h}}\right)^{2}$. E também:

$$
\Gamma\left(h \rightarrow Z^{0} Z^{0}\right)=\frac{G_{F} m_{h}^{3}}{16 \pi \sqrt{2}}\left(1-4 \lambda_{Z}\right)^{1 / 2}\left(12 \lambda_{Z}^{2}-4 \lambda_{Z}+1\right)
$$

onde $\lambda_{Z}=\left(\frac{m_{Z}}{m_{h}}\right)^{2}$.

Para

$$
m_{h} \gg m_{Z} \Rightarrow \lambda_{W} \sim \lambda_{Z} \Rightarrow \frac{\Gamma\left(h \rightarrow Z_{0} Z_{0}\right)}{\Gamma\left(h \rightarrow W^{+} W^{-}\right)} \sim \frac{1}{2} .
$$

Considerando-se separadamente os casos em que os bosons vetoriais produzidos sejam transversal $(\mathrm{T})$ ou longitudinalmente $(\mathrm{L})$ polarizados, as taxas de decaimento obtidas são [1]:

- para polarização transversal ( \pm ):

$$
\Gamma\left(h \rightarrow W_{(+)}^{+} W_{(+)}^{-}\right)=\Gamma\left(h \rightarrow W_{(-)}^{+} W_{(-)}^{-}\right)=\frac{g^{2}}{16 \pi} \frac{m_{W}^{2}}{m_{h}}\left(1-\frac{4 m_{W}^{2}}{m_{h}^{2}}\right)^{1 / 2}
$$


- para polarização longitudinal:

$$
\Gamma\left(h \rightarrow W_{L}^{+} W_{L}^{-}\right)=\frac{g^{2}}{64 \pi} \frac{m_{h}^{3}}{m_{W}^{2}}\left(1-\frac{2 m_{W}^{2}}{m_{h}^{2}}\right)^{2}\left(1-\frac{4 m_{W}^{2}}{m_{h}^{2}}\right)^{1 / 2}
$$

Um detalhe fundamental é a dependência da largura do decaimento em bosons longitudinais com o cubo da massa do higgs. Como a largura para bosons longitudinais é dominante sobre a largura para bosons transversais (será mostrado em seguida), isso implica em dificuldades na deteção de um higgs pesado.

Das equações acima observa-se que para $m_{h} \gg m_{V}, V=W$ ou $Z$, o decaimento é predominantemente em bosons polarizados longitudinalmente:

$$
\frac{\Gamma\left(h \rightarrow V_{T}^{+} V_{T}^{-}\right)}{\Gamma\left(h \rightarrow V_{L}^{+} V_{L}^{-}\right)} \sim \frac{\frac{1}{2}\left(\frac{2 m_{V}}{m_{h}}\right)^{4}}{\left[1-\frac{1}{2}\left(\frac{2 m_{V}}{m_{h}}\right)^{2}\right]^{2}} \sim \frac{8 m_{V}^{4}}{m_{h}^{4}},
$$

enquanto os bosons produzidos no fundo contínuo é predominantemente polarizado transversalmente. Por algum tempo pensou-se em usar essa diferença como assinatura para o higgs, mas no momento essa idéia está descartada pelas dificuldades experimentais em se detetar essa diferença.

Uma outra razão interessante é a obtida para decaimentos do higgs em dois fotons e em dois W's:

$$
\frac{\Gamma(h \rightarrow \gamma \gamma)}{\Gamma\left(h \rightarrow W^{+} W_{-}\right)} \approx \frac{1}{4}\left(\frac{\alpha}{\pi}\right)^{2} \approx 10^{-6} .
$$

\subsection{Modos de Produção}

Como o higgs se acopla muito fracamente com os constituintes dos feixes tanto em anéis de colisão de hadrons como de eletrons, a sua deteção experimental torna-se extremamente difícil, sendo necessário analisar cuidadosamente todas as opções disponíveis. Nessa seção serão abordados os principais modos de produção do higgs, com maior ênfase nos modos predominantes em anéis de colisão hadrônicos com grande energia no centro de massa $(\sim 10-40 \mathrm{TeV})$. A fig.(3.6) mostra uma comparação entre as seções 


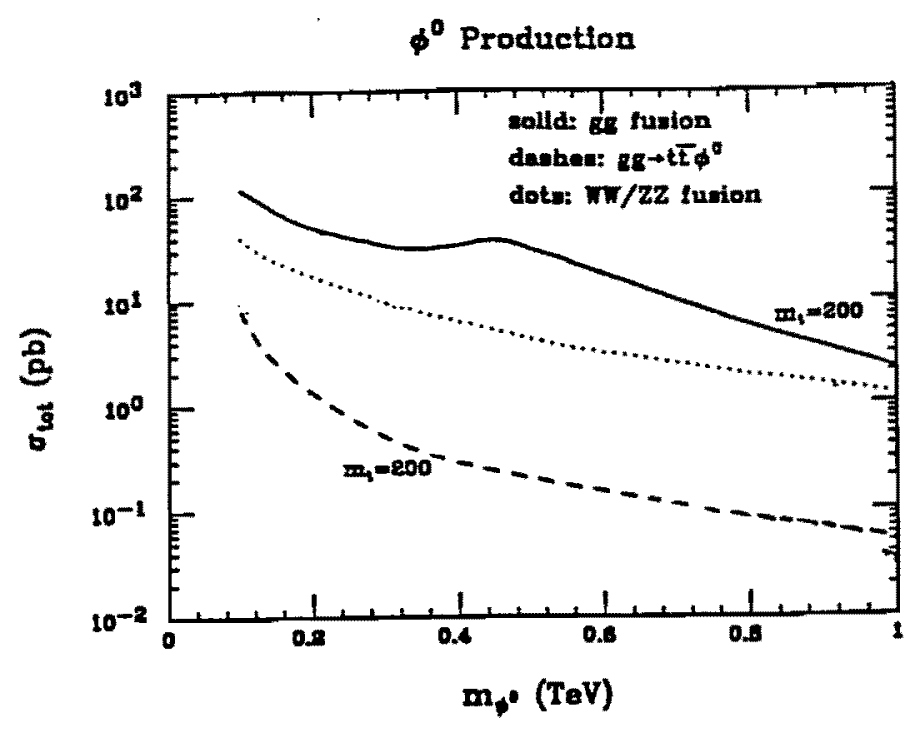

Figura 3.6: Comparação entre as seções de choque para a produção de higgs por fusão de W's e por fusão de gluons. Figura retirada da ref. [27].

de choque para a produção de higgs por fusão e W's e por fusão de gluons. A massa do top é considerada como sendo $m_{t}=200 \mathrm{GeV}$. Observa-se que para essa região de massa do top, que não produz resultados muito diferentes que paraa massa real, a fusão de gluons é o modo de produção dominante .

\subsubsection{Fusão de Fermions}

A fig.( 3.7) mostra a produção do boson de Higgs a partir da fusão de um fermion com um anti-fermion. Esse modo de produção é bastante suprimido quando comparado aos demais que serão apresentados a seguir, porque o acoplamento do higgs com os fermions é proporcional a $\frac{m_{f}}{m_{W}}$, como já foi diversas vezes mencionado.

Em particular, a fusão $e^{+} e^{-} \rightarrow h$, num anel de colisão eletron-positron tem seção de choque muito inferior à radiação de um higgs por um $Z$, que será descrita adiante.

Num anel de colisão hadrônico, poderia-se esperar que a fusão de pares de tops pertencentes ao mar dos protons incidentes, atingisse uma taxa de 


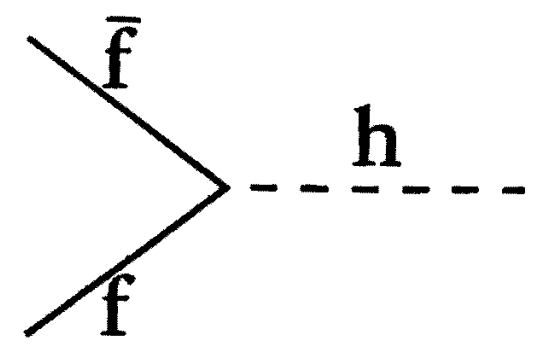

Figura 3.7: Produção do boson de Higgs por fusão de um par fermion antifermion.

eventos aceitável, devido à grande massa do top. Isso não ocorre, porém, porque a probabilidade de se encontrar um top no mar de quarks do proton é praticamente nula.

\subsubsection{Bremsstrahlung de $Z$}

Esse modo de produção é especialmente importante em anéis de colisão $e^{+} e^{-}$, onde é grande a produção de Z's no canal s. A fig. (3.8) mostra o diagrama correspondente, para dois casos.

No primeiro deles o $Z$ produzido no canal $s$ está na camada de massa, e decai em um $h$ e num $Z^{*}$ ( $Z$ virtual). Foi proposto inicialmente por Ioffe e Khoze [36] e posteriormente calculado por Björken [20]. É o mecanismo dominante em colisões $e^{+} e^{-}$que ocorrem próximas da energia de ressonância do $Z$ e os limites experimentais apresentados na sec.(3.1.2) foram obtidos após a análise desse tipo de reação no LEP I.

A largura diferencial para a de integração $Z^{0} \rightarrow l^{+} l^{-} h$ é:

$$
\frac{1}{\Gamma(Z \rightarrow l \bar{l})} \frac{d \Gamma}{d x}(Z \rightarrow l \bar{l} h)=\frac{\alpha\left(1-x+\frac{x^{2}}{12}+\frac{2}{3} y^{2}\right)\left(x^{2}-4 y^{2}\right)^{1 / 2}}{4 \pi x_{W}\left(1-x_{W}^{2}\right)(x-y)^{2}}
$$

onde $x=2 E_{h} / m_{Z}=\left(m_{h}^{2}+m_{Z}^{2}-m_{l i}^{2}\right)$ e $y=m_{h} / m_{Z}$. 


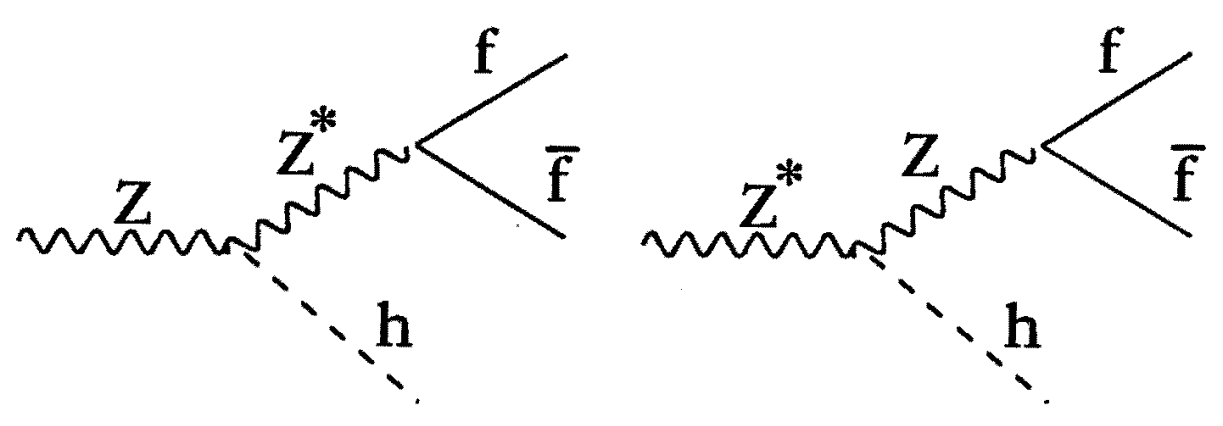

Figura 3.8: Diagrama com um higgs sendo radiado por um $Z^{0}$ real ou virtual.

No segundo caso é produzido um $Z$ virtual no canal $s$, que posteriormente emite um higgs (parte b da figura), voltando dessa forma, à camada de massa. Esse processo deve ser importante em colisões em que a energia no centro de massa seja superior à massa do $Z$, como será o caso do LEP II.

Nesse caso a seção de choque é dada por [37]:

$$
\sigma\left(e^{+} e^{-} \rightarrow Z^{0} h\right)=\frac{G_{F}^{2} m_{Z}^{4}}{96 \pi}\left[1+\left(1-4 x_{W}\right)^{2}\right] \frac{8 k}{\sqrt{s}}\left[\frac{k^{2}+3 m_{Z}^{2}}{\left(s-m_{Z}^{2}\right)^{2}}\right],
$$

onde $k$ é o momento do higgs no centro de massa (ou do $Z$ produzido).

Mesmo num anel de colisão hadrônico pode haver produção do higgs por radiação, através do processo $q \bar{q} \rightarrow Z h$. Embora tenha uma pequena seção de choque, a assinatura é bastante limpa.

Em todos os casos, o sinal procurado é o decaimento do $Z$ em um par de leptons (eletrons ou muons). Podem ser usados também os canais $Z \rightarrow \nu \bar{\nu} \mathrm{e}$ no caso de anéis $e^{+} e^{-}$, mesmo o decaimento $Z \rightarrow q \bar{q}$ pode ser considerado.

\subsubsection{Bremsstrahlung de $W^{ \pm}$}

Em anéis de colisão hadrônicos o processo $q \bar{q} \rightarrow W^{ \pm} h$ tem uma seção de choque pequena, mas com uma assinatura clara se é considerado o canal $W^{ \pm} \rightarrow l \nu$, em que o lepton é duro e isolado e pode ser usado para tag. 


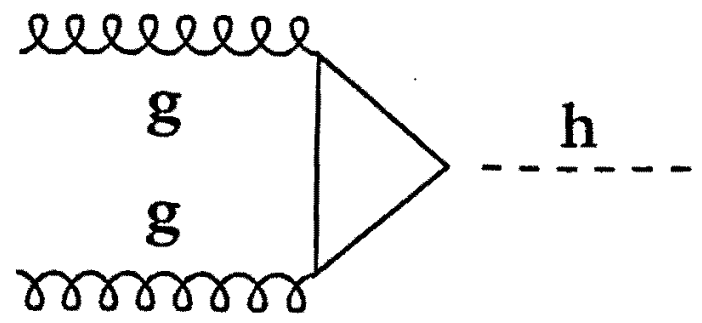

Figura 3.9: Diagrama para a produção de higgs por fusão de gluons.

\subsubsection{Fusão de Gluons}

Em colisões $p p$ ou $p \vec{p}$ a fusão de gluons é o modo dominante de produção de higgs, que ocorre através de um loop de fermions pesados, conforme a fig. (3.9). Embora não seja um diagrama de primeira ordem, é bastante freqüente devido à grande quantidade de gluons existente no proton.

A expressão geral para a seção de choque para produção de uma única partícula $P$ de masssa $M$, spin $J$ e $n_{c}$ graus de liberdade de cor numa colisão entre os hadrons $A$ e $B$ [1] é:

$$
\begin{aligned}
\sigma(A B \rightarrow P+X)= & \frac{16 \pi^{2}(2 J+1) n_{c}}{M^{3}} \\
& K \sum_{a, b} C_{a b} r_{a b} S \Gamma(P \rightarrow a b) \tau \\
& \int_{\tau}^{1} \frac{d x}{x}\left[f_{a / A}\left(x, M^{2}\right) f_{b / B}\left(\tau / x, M^{2}\right)+(A \leftrightarrow B \text { se } a \neq b)\right],
\end{aligned}
$$

onde:

- $S$ é um fator estatístico, igual a 2 para singletos de cor $(P \rightarrow \gamma \gamma, g g, Z Z$, bosons idênticos) e igual a 1 nos outros casos.

- $K$ é um fator que representa correções de QCD para ordens não liderantes $\left(K \simeq 1.5\right.$ para $g g \rightarrow h[38]$ e $K \simeq 1.15$ para $q \vec{q} \rightarrow W^{ \pm} h, Z h[39]$. 
- $\tau=M^{2} / s$.

- $f_{a / A}\left(x, M^{2}\right)$ é a função de distribuição de partons calculada na escala $M$.

- $r_{a b}$ é um fator que introduz uma média feita sobre os spins (para quarks e gluons, $\left.r_{a b}=\frac{1}{4}\right)$.

- O fator $C_{a b}$ depende do número de cores possíveis para os partons $\left(C_{q \bar{q}}=\frac{1}{g}, C_{q g}=\frac{1}{24}\right.$ e $\left.C_{g g}=\frac{1}{64}\right)$.

Para a produção de higgs por fusão de gluons, a expressão acima fica:

$$
\sigma\left(p p \rightarrow g g+X \rightarrow h+X^{\prime}\right)=\Gamma(h \rightarrow g g) \frac{\pi^{2}}{8 m_{h}^{3}} \tau \int_{\tau}^{1} \frac{d x}{x} g\left(x, m_{h}^{2}\right) g\left(\frac{\tau}{x}, m_{h}^{2}\right),
$$

onde $\tau=\frac{m_{h}^{2}}{s}$ e $g\left(x, Q^{2}\right)$ é a função de distribuição de gluons calculada na escala de massa $Q^{2}$. Substituindo a eq. (3.28) com a aproximação de $I=N$ (sendo $N$ o número de quarks pesados), chega-se a:

$$
\sigma\left(p p \rightarrow g g+X \rightarrow h+X^{\prime}\right)=\frac{G_{F}}{\sqrt{2}}\left(\frac{\alpha_{s}}{3 \pi}\right)^{2} \frac{\pi N^{2}}{32} \tau \int_{\tau}^{1} \frac{d x}{x} g\left(x, m_{h}^{2}\right) g\left(\frac{\tau}{x}, m_{h}^{2}\right) .
$$

\subsubsection{Fusão de W's ou de Z's}

Se a massa do top fosse menor do que é na realidade, algo em torno de 100 $\mathrm{GeV}$, haveria um valor da massa do higgs a partir do qual a produção do higgs seria mais provável por fusão de bosons vetoriais longitudinais. Porém, com a massa que ele possui, a produção por fusão de gluons domina em todo o espectro de massa do higgs.

Ainda assim é muito importante analisar esse processo por dois motivos. O primeiro dele é que a fusão de bosons vetoriais tem uma seção de choque que ainda merece atenção, embora não seja a maior de todas. O segundo, e mais importante é o fato da interação do modo longitudinal dos bosons vetoriais com o higgs ser uma das formas mais diretas de testar o setor de quebra de simetria (pag. 12). 

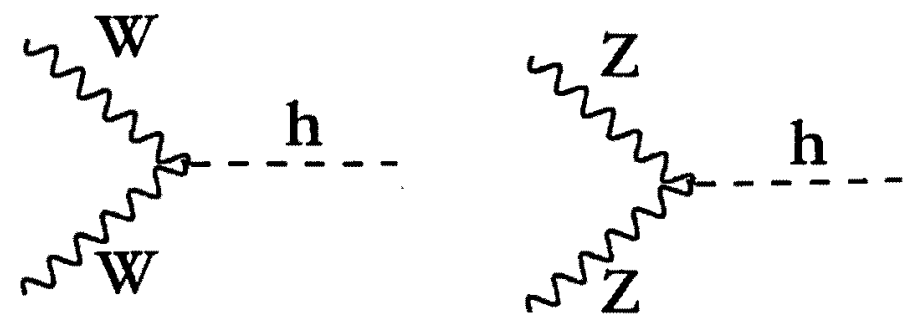

Figura 3.10: Fusão. WW e ZZ para a produção de bosons de Higgs.

\section{Cálculo Exato}

$\mathrm{Na}$ fusão de W's ou de Z's quarks pertencentes aos feixes incidentes emitem bosons virtuais $\mathrm{W}$ ou $\mathrm{Z}$ que se aniquilam para formar um higgs. Os dois processos estão apresentados na fig. (3.10).

$\mathrm{O}$ elemento de matriz para o subprocesso $q_{1} q_{2} \rightarrow q_{1}^{\prime} q_{2}^{\prime} V_{1} V_{2} \rightarrow q_{1}^{\prime} q_{2}^{\prime} h$ é $[1]$, [40], [41]:

$$
\mathcal{A}=C_{V} \bar{u}\left(q_{1}^{\prime}\right) \gamma^{\mu}\left(g_{V}^{q_{1}}+g_{A}^{q_{1}} \gamma_{5}\right) u\left(q_{1}\right) \bar{u}\left(q_{2}^{\prime}\right) \gamma_{\mu}\left(g_{V}^{q_{2}}+g_{A}^{q_{2}} \gamma_{5}\right) u\left(q_{2}\right) D_{1} D_{2},
$$

onde:

- $V$ representa qualquer um dos bosons vetoriais com massa, $W^{ \pm}$ou $Z$.

- Para bosons $W$ :

1. $C_{W}=g^{3} m_{W}$

2. $g_{V}^{q}=-g_{A}^{q}=\frac{1}{2 \sqrt{2}}$

- Para bosons Z:

1. $C_{Z}=g^{3} m_{W} /\left(1-x_{W}\right)^{2}$ 
2. $g_{V}^{q}=\frac{1}{4}-\frac{2}{3} x_{W}$ e $g_{A}=-\frac{1}{4}$ se $q=u, c, t$

3. $g_{V}^{q}=-\frac{1}{4}+\frac{1}{3} x_{W}$ e $g_{A}=\frac{1}{4}$ se $q=d, s, b$

- $D_{i}=\left(V^{2}-m_{V}^{2}+i m_{V} \Gamma_{V}\right)^{-1}$, onde o nome da partícula é usado para denotar seu quadrimomento.

Portanto o elemento de matriz ao quadrado, somado e feita a média sobre os spins dos quarks iniciais, é:

$$
\sum|\mathcal{A}|^{2}=4 C_{V}^{2}\left[G_{1} q_{1} \cdot q_{2} q_{1}^{\prime} \cdot q_{2}^{\prime}+G_{2} q_{1} \cdot q_{2}^{\prime} q_{1}^{\prime} \cdot q_{2}\right]\left|D_{1}\right|^{2}\left|D_{2}\right|^{2}
$$

onde $G_{1}=\left(g_{L}^{q_{1}}\right)^{2}\left(g_{L}^{q_{2}}\right)^{2}+\left(g_{R}^{q_{1}}\right)^{2}\left(g_{R}^{q_{2}}\right)^{2}, G_{2}=\left(g_{L}^{q_{1}}\right)^{2}\left(g_{R}^{q_{2}}\right)^{2}+\left(g_{R}^{q_{1}}\right)^{2}\left(g_{L}^{q_{2}}\right)^{2}$, com $g_{L, R}=g_{V} \mp g_{A}$.

Para se levar adiante um cálculo quase exato, que descreve um estado final com dois quarks e um higgs, é necessário descrevê-los em termos de três ângulos de Euler. Ignorando a dependência azimutal da direção do feixe inicial, trabalha-se com somente dois ângulos, e duas energias. No centro de massa do subprocesso (ou seja, no centro de massa dos dois quarks incidentes), a seção de choque é dada por:

$$
d \hat{\sigma}=\frac{(2 \pi)^{-4}}{16 \hat{s}}|\mathcal{A}|^{2} d E_{1}^{\prime} d E_{2}^{\prime} d \alpha d \cos \beta,
$$

onde $E_{1}^{\prime}$ e $E_{2}^{\prime}$ são as energias dos quarks finais. Os ângulos $\alpha$ e $\beta$ são mostrados na fig. (3.11). $\theta$, que é mostrado na mesma figura, é o ângulo entre $p_{1}^{\prime} \mathrm{e}$ $-p_{2}^{\prime}$, onde $p_{1}^{\prime}$ e $p_{2}^{\prime}$ são os momentos dos quarks finais. $\hat{s}$ é a energia no centro de massa do subprocesso, $\hat{s}=\left(p_{1}+p_{2}\right)^{2}$.

Fazendo a parametrização:

$$
E_{1}^{\prime}=\frac{\sqrt{\hat{s}}}{2}(1-\eta) \text { e } E_{2}^{\prime}=\frac{\sqrt{\hat{s}}}{2}(1-\zeta),
$$

chega-se a:

$$
d \hat{\sigma}=\frac{C A^{2}}{2^{10} \pi^{4} \hat{s}^{2}} \frac{2(1+\cos \theta)}{(1-\zeta)(1-\eta)} J\left(1+\frac{2 m_{W}^{2}}{(1-\eta) \hat{s}}, 1+\frac{2 m_{W}^{2}}{(1-\zeta) \hat{s}}, \theta\right) d \eta d \zeta,
$$

com $J(x, y, \theta)$ sendo dada pela expressão: 


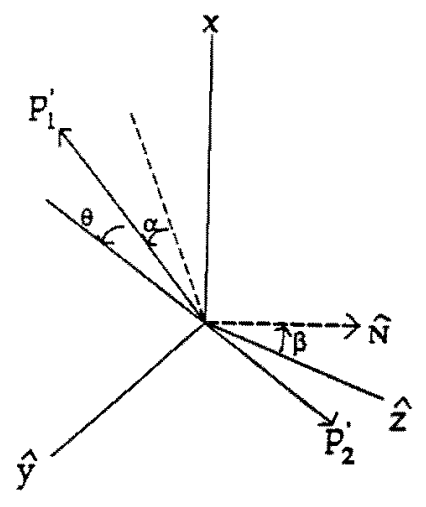

Figura 3.11: Cinemática da fusão WW. A orientação do plano que contém o estado final é especificado pelos ângulos $\beta$ que a normal ao plano $\hat{N} \mathrm{faz}$ com o eixo $z$ e pelo ângulo $\alpha$ que fica no referido plano. $\mathrm{O}$ ângulo entre $p_{1}^{\prime} \mathrm{e}-p_{2}^{\prime}$ é $\theta$.

$$
\begin{aligned}
J(x, y, \theta)= & \int_{0}^{2 \pi} d \alpha \int_{-1}^{1} d \cos \beta(x+\cos \alpha \sin \beta)^{-2} \times(y+\cos (\alpha+\theta) \sin \beta)^{-2} \\
= & 4 \pi\left[\frac{3}{\Delta^{2}}\left(\frac{1}{\sqrt{\Delta}} \tanh ^{-1} \frac{\sqrt{\Delta}}{x y-\cos \theta}-\frac{x y-\cos \theta}{\left(x^{2}-1\right)\left(y^{2}-1\right)}\right)\right] \times \\
& (x-y \cos \theta)(y-x \cos \theta) \frac{\cos \theta}{\Delta^{3 / 2}} \tanh ^{-1} \frac{\sqrt{\Delta}}{x y-\cos \theta}+ \\
& \frac{x^{2}+y^{2}-3 x y \cos \theta+1}{\Delta\left(x^{2}-1\right)\left(y^{2}-1\right)}
\end{aligned}
$$

sendo $\Delta=x^{2}+y^{2}-2 x y \cos \theta-1+\cos ^{2} \theta$.

Daqui em diante é necessário realizar uma integraçăo numérica para prosseguir.

\section{Aproximação de W Efetivo}

No limite de altas energias, as massas dos bosons vetoriais ficam cada vez menos importantes, de forma que o processo assemelha-se a uma colisão $\gamma \gamma$ num anel de colisão $e^{+} e^{-}$, que é usualmente tratada pela aproximação de 
foton equivalente (ou aproximação de Weizacker-Williams [42]). Os fotons são tratados como reais (e portanto necessariamente transversais) e colineares aos os feixes incidentes. Para o processo $V V \rightarrow h$, o análogo cinemático são os quarks emitindo os W's por bremsstrahlung, sem que com isso sofram desvio. São produzidos tanto W's longitudinais como transversais. O espalhamento sem mudança de direção corresponde aos limites:

$$
\cos \alpha \rightarrow-1, \quad \sin \beta \rightarrow+1, \quad \theta \rightarrow 0,
$$

ou seja [41],

$$
\hat{\sigma}=\frac{C A^{2}}{2^{10} \pi^{3} m_{W}^{4}} \int_{m_{h}^{2}}^{1} \int_{m_{h}^{2} / \hat{s} \eta}^{1+\left(m_{h}^{2} / \hat{s}\right)-\eta} d \zeta(1-\zeta)(1-\eta) \delta\left(\zeta \eta-m_{h}^{2} / \hat{s}\right) .
$$

Em particular, para o processo $W W \rightarrow h, A=g m_{W}$ e $C=g^{4}=$ $(4 \pi \alpha)^{2} / \sin ^{4} \theta_{W}$, e a seção de choque fica:

$$
\hat{\sigma}=\frac{1}{16 m_{W}^{2}}\left(\frac{\alpha}{\sin ^{2} \theta_{W}}\right)^{3}\left(\left(1+m_{h}^{2} / \hat{s}\right) \log \left(\hat{s} / m_{h}^{2}\right)-2+2 m_{h}^{2} / s\right) .
$$

Dawson [43], partindo da eq. (3.54) obteve a distribuição de W's nos quarks:

$$
\sigma_{e f f}=\left.\frac{16 \pi^{2}}{m_{h}^{3}} \Gamma\left(h \rightarrow W^{+} W^{-}\right) \tau \frac{d \mathcal{L}}{d \tau}\right|_{p p / W W}
$$

onde

$$
\left.\frac{d \mathcal{L}}{d \tau}\right|_{p p / W W}=\int_{\tau}^{1} f_{q / W}(x) f_{q / W}\left(\frac{\tau}{x}\right) \frac{d x}{x}
$$

é a luminosidade de bosons $\mathrm{W}$ num sistema de dois quarks. 


\section{Capítulo 4}

\section{Colisões Hadrônicas}

Neste capítulo serão revistos alguns aspectos das colisões hadrônicas que são essenciais para a análise dos resultados apresentados nessa tese. O objetivo não é fazer uma revisão ampla ou profunda acerca do assunto, mas somente introduzir alguns conceitos elementares da física de colisões de hadrons.

$\mathrm{Na}$ colisão entre dois hadrons a altas energias, vários tipos de interações podem ocorrer. As menos freqüentes, porém mais fáceis de se lidar, são colisões inelásticas, como as interações eletrofracas, e as fortes em que um grande momento transversal é trocado. Nesses casos pode-se fazer uma expansão perturbativa para calcular sua amplitude de espalhamento. Nas colisões elásticas, nas difrativas e na maioria das inelásticas o momento trocado é pequeno, não sendo possível usar apenas os termos de ordens mais baixa de uma expansão da amplitude em termos da constante de acoplamento, embora não haja dúvida de que é a QCD que rege essas interações. As interações que se encaixam nesse último caso serão genericamente tratadas por minimum bias nesse trabalho.

\subsection{Seção de Choque Total}

$\mathrm{Na}$ interação entre dois hadrons, a seção de choque total é dada por:

$$
\sigma_{T}=\sigma_{d i f}+\sigma_{n d},
$$

onde estão representadas as seções de choque total $\left(\sigma_{T}\right)$, difrativa $\left(\sigma_{\text {dif }}\right)$ e não difrativa $\left(\sigma_{n d}\right)$. 
Um processo difrativo caracteriza-se por não haver troca de números quânticos entre os hadrons envolvidos e, no caso difrativo inelástico, pelo fato dos produtos da colisão localizarem-se na direção dos feixes incidentes. $\mathrm{Na}$ maior parte dos eventos, é possivel identificar no estado final um ou os dois dos hadrons iniciais (às vezes em algum estado excitado). Eles podem ser elásticos ou inelásticos, e os inelásticos podem constituir uma difração simples, dupla ou dura.

$$
\sigma_{d i f}=\sigma_{e l}+\sigma_{d i f_{i n}} \quad \text { e } \quad \sigma_{d i f_{i n}}=\sigma_{s d}+\sigma_{d d}+\sigma_{d h}
$$

onde estão representadas as seções e choque difrativa elástica $\left(\sigma_{e l}\right)$, inelástica $\left(\sigma_{d i f_{i n}}\right)$, difração simples $\left(\sigma_{s d}\right)$, difração dupla $\left(\sigma_{d d}\right)$ e difração dura $\left(\sigma_{d h}\right)$.

\subsubsection{Processos Difrativos}

A maior parte dos processos difrativos é constituída de interações com pequeno momento transversal transferido, de forma que não são bem descritos pela QCD perturbativa. Os modelos que melhor reproduzem os dados experimentais no momento são baseados em troca de pomerons.

\section{Processos Difrativos Elásticos}

São processos em que os dois hadrons iniciais são identificados no estado final.

Há um limite superior para a razão $\sigma_{e l} / \sigma_{T}$, conhecido como limite de Pumplin [51], obtido a partir de argumentos de unitariedade:

$$
\frac{\sigma_{e l}}{\sigma_{T}} \leq \frac{1}{2}
$$

A fig. (4.1) mostra as seções de choque total e elástica [22] em função da energia no centro de massa, para colisões proton-proton, onde se vê que a relação(4.1.1) é confortavelmente satisfeita.

\section{Espalhamento Difrativo Simples}

No espalhamento difrativo simples um dos hadrons iniciais emerge no estado final praticamente com o mesmo quadrimomento com que iniciou o processo e somente um pouco defletido; quando ocorre a energias características de 
CAPÍTULO 4. COLISÕES HADRÔNICAS

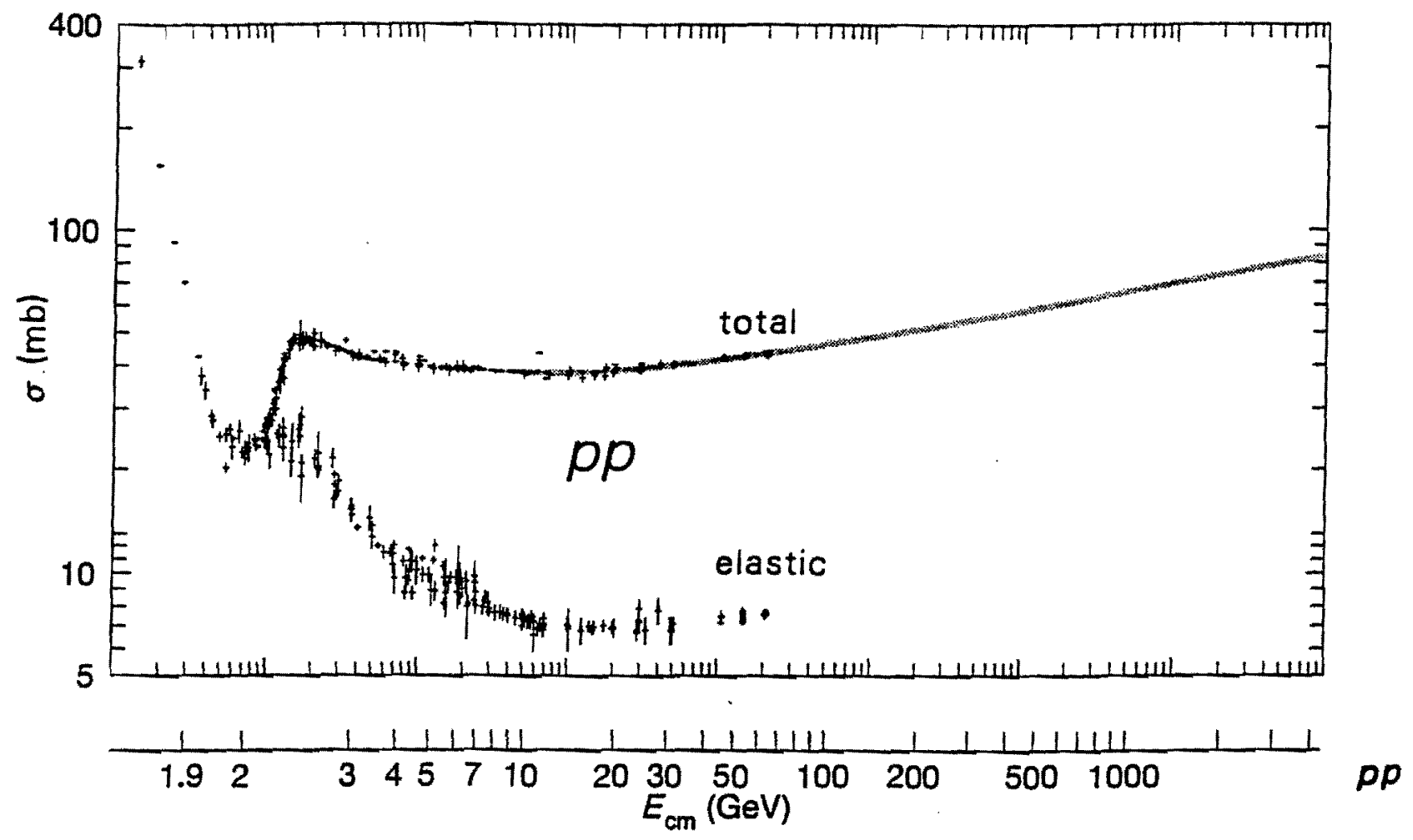

Figura 4.1: Seção de choque total e elástica em função da energia no centro de massa para colisões proton-proton [22]. 


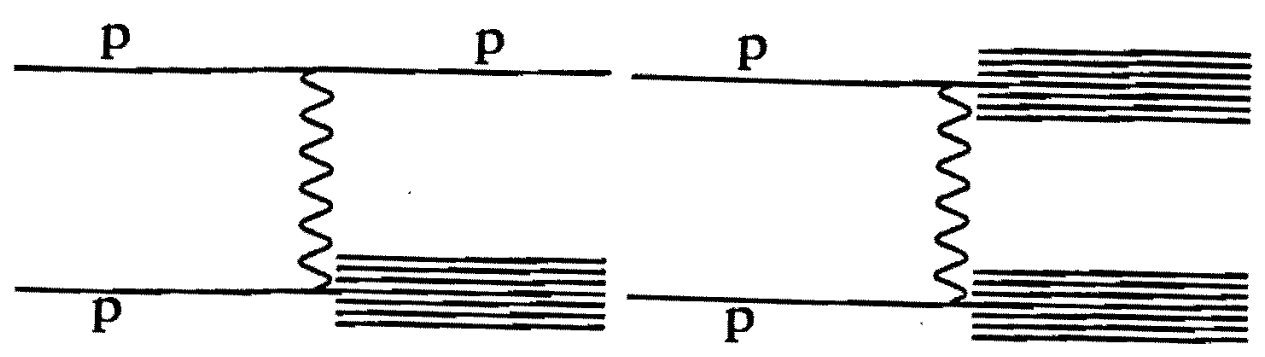

Figura 4.2: Esquema para processos difrativos.

a) difração simples; b) difração dupla.

colliders, forma-se um gap de rapidez (uma região sem partículas no espaço de rapidez) entre o hadron difratado e as outras partículas do estado final. Aproximadamente $20 \%$ dos processos que ocorrem a essas energias numa colisão $p p$ são difrações simples [50]. A fig. (4.2a) mostra esquematicamente uma difração simples.

\section{Espalhamento Difrativo Duplo}

Neste caso, nenhum dos dois hadrons iniciais é observado na análise final do processo. Espera-se um gap de rapidez entre dois grupos de partículas, como sinal característico. A fig. (4.2b) mostra esquematicamente uma difração dupla. A seção de choque para as difrações duplas é [50]:

$$
\sigma_{d d} \approx 1.3 \frac{\sigma_{s d}^{2}}{\sigma_{e l}} \approx 0.05 \sigma_{T}
$$

Tanto na difração simples como na dupla, as partículas finais encontramse colimadas. Como o momento transferido é pequeno, cada grupo de partículas apresenta momento, energia e direção próximos aos dos feixes iniciais. Há uma generalização do limite de Pumplin (eq. (4.1.1), devido a Sukhatme e Henyey [52]: 


$$
\frac{\sigma_{s d}+\sigma_{d d}}{\sigma_{T}} \leq \frac{1}{2}
$$

\section{Processos Difrativos Duros}

Ingelman e Schlein [47] sugeriram a existência de um espalhamento difrativo duro, cujo estado final contém, além dos produtos de uma difração, os produtos de um espalhamento duro, como por exemplo jatos, um quark pesado, ou um par Drell-Yan. Para o cálculo da seção de choque aplica-se o formalismo usual de espalhamento duro a um sistema onde o alvo é um pomeron, tratado nesse caso como uma partícula quase real. Em ordem mais baixa de $\alpha_{s}$, a expressão utilizada seria:

$$
\sigma(A+\text { pomeron } \rightarrow j \text { atos }+X)=\sum_{a, b} f_{a / A}\left(x_{a}\right) f_{b / \mathcal{P}}(\beta) \hat{\sigma}(a+b \rightarrow j a t o s),
$$

onde $a$ é o parton do hadron $A$ que vai colidir com o parton $b$ do pomeron; $f_{a / A}$ é a função de distribuição do parton $a$ no hadron $A$ e $f_{b / \mathcal{P}}$ é a função de distribuição do parton $b$ no pomeron, $x_{A}$ é a fração de momento que o parton $a$ toma do hadron $A$ e $\beta$ é a fração de momento que o parton $b$ toma do pomeron.

A observação desse tipo de colisões difrativas, que permitiria o estudo de uma possível função de distribuição de partons no pomeron, foi reportada pela experiência UA8 [48], e as seções de choques obtidas (com determinados ansatz para a distribuição de quarks e gluons no pomeron) concordam em ordem de grandeza com os dados experimentais. Estas observações foram posteriormente confirmadas pelo HERA [99]. A fig. (4.3) mostra um espalhamento duro difrativo.

Collins, Frankfurt e Strikman, analisando esse tipo de reação [49] concluíram que há outras contribuições além das que são levadas em conta por Ingelman e Schlein (fig. (4.4)), e chamou-as de espalhamento difrativo duro coerente, onde o pomeron como um todo induz uma difração dura.

Será comentado adiante (pag. (57)) que essa nova visão da difração traz algumas consequëncias importantes para a análise das seções de choque inclusivas, pois os teoremas de fatoração normalmente utilizados perdem sua validade no caso das colisões difrativas duras. 


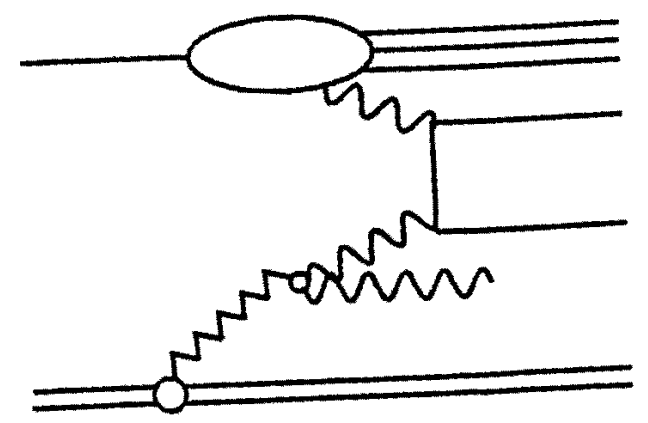

Figura 4.3: Espalhamento Difrativo Duro segundo o modelo de IngelmanSchlein [47](figura retirada da ref. [49]).

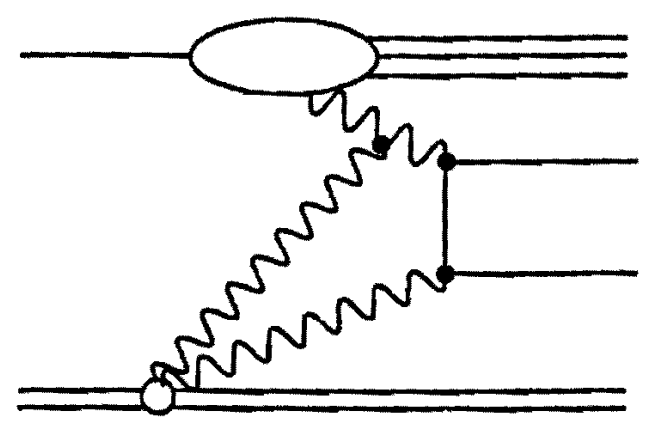

Figura 4.4: Espalhamento Difrativo Duro Coerente (figura retirada da ref. [49]) 


\subsubsection{Processos Não Difrativos}

Os processos não difrativos são sempre inelásticos. Podem ser soft ou duros. No primeiro caso, sua descrição depende de modelo, por ter pequeno momento transversal trocado, e portanto não ser passível de descrição por teoria de perturbações. Dois desses modelos serão usados nesse trabalho, e estão descritos no próximo capítulo, na sec.(5.1.2), sendo que um faz parte da abordagem do PYTHIA, e outro da abordagem do DTUJET.

Os processos não difrativos duros incluem as colisões eletrofracas e as colisões fortes com grande momento transversal transferido (duras). $O$ cálculo da seção de choque para essas reações é feito através de uma expansão perturbativa da amplitude de espalhamento em função da constante de acoplamento. As ref.[53, 54] tratam do assunto de forma completa. O processo inelástico duro pode ser fatorado, e tratado separadamente do processo subjacente.

Toda a teoria desenvolvida até agora parte do pressuposto de que é possível escrever a seção de choque inclusiva de um processo hadrônico duro na forma fatorada (isto é, usando o modelo a partons):

$\sigma\left(A+B \rightarrow c+X+X^{\prime}\right)=\int_{0}^{1} d x_{1} \int_{0}^{1} d x_{2} f_{a / A}\left(x_{1}, Q^{2}\right) f_{b / B}\left(x_{2}, Q^{2}\right) \hat{\sigma}(a+b \rightarrow c)$

sendo $x_{1}, x_{2}$ respectivamente as frações de quadrimomento que os partons iniciais a e b retiraram dos hadrons a que pertenciam, e $Q^{2}$ a escala de momento em que ocorre a reação. As funções de distribuição $f_{i / h}\left(x_{i}, Q^{2}\right)$, dão a distribuição de probabilidade de se encontrar o parton $i$ dentro do hadron inicial $h$, carregando uma fração de momento do hadron inicial entre $x_{i}$ e $x_{i}+d x_{i}$.

\subsubsection{Fatoração}

Fatorar uma seção de choque implica em que se esteja assumindo duas coisas:

- É possível separar a interação dos dois partons duros do resto dos hadrons, ou seja, há um momento a partir do qual os quarks ativos na colisão dura não reagem mais com os hadrons de que se originaram. Pode-se portanto escrever para esse dado processo a função de distribuição de partons dentro do hadron, $f\left(x, Q^{2}\right)$. Isso é a fatoração em si. 
- Supõe-se, além disso, que essa função de distribuição dos partons dentro do hadron seja universal, isto é, não dependa do processo. Seria possível, portanto obter experimentalmente as funções de distribuição com o espalhamento inelástico profundo, e utilizá-las para os demais processos hadrônicos.

Não é óbvio que qualquer um desses dois pontos valham sempre, podendose, para cada processo, distinguir três possibilidades:

- a fatoração vale, e as funções de distribuição em qualquer processo são as obtidas no espalhamento inelástico profundo.

- a fatoração vale, mas as funções de distribuição dependem do processo em questão.

- não é possível fatorar, ou seja, não é possivel separar o processo duro da evolução dos feixes restantes.

Esse tema foi abordado inicialmente em 1974 por Cardy e Winbow [55], que mostraram que a seção de choque inclusiva para um processo Drell-Yan $\left(q \bar{q} \rightarrow \mu^{+} \mu^{-}\right)$é independente do fato dos diquarks remanescentes dos dois protons trocarem ou não um pomeron entre si, o que justificava a hipótese de Feynman (modelo a partons) [75] de que as interações das wee particles não afetam a distribuição dos partons duros. No ano seguinte De Tar, Ellis e Landshoff [56] estenderam essa demonstração para qualquer número de pomerons trocados. Esses dois trabalhos são anteriores aos desenvolvimentos de QCD.

As publicações posteriores, no contexto da QCD [58, 59, 57,60], abordam o problema de outra forma. Além da interação dura que se está analisando, podem ocorrer outras reações, iniciais ou finais, entre os partons espectadores, ou entre os partons ativos e os espectadores, e é preciso verificar se elas não interferem na possibilidade de fatoração, ou não tornam a função de distribuição de partons no hadron dependente do processo. Quanto às interações finais, que ocorrem após a interação dura, parte-se do princípio de que não podem reagir retroativamente, e portanto não interferem na seção de choque para a interação dura.

Uma possível interação inicial é a que ocorre entre os quarks de um dos hadrons iniciais. Por exemplo, pode-se imaginar que um quark vermelho 
possa ser salvo da aniquilação num processo Drell-Yan através da emissão de um gluon, o que mudaria a sua cor. Já no espalhamento inelástico profundo, isso não muda a seção de choque. Seria de se supor nesse caso que as funções de distribuição fossem diferentes num caso e no outro. Assumir que a fatoração vale, implica em assumir que há um cancelamento das trocas de cor em ordem dominante. No modelo a partons, a justificativa para esse cancelamento é razoavelmente simples. O proton está a uma velocidade relativística, e portanto seu tempo interno se dilata. Isso implica que, comparado com a escala de tempo em que ocorre uma colisão dura $\tau \sim \sqrt{Q^{-2}}$ sendo $Q$ o momento transferido, a configuração interna do proton é estacionária, e se houver interação entre os quarks do hadron, ela ocorre muito tempo antes da colisão dura. Porém, considerando-se a QCD, a questão se complica um pouco, pois um quark pode emitir um gluon com grande $p_{T}$, que portanto pode ocorrer próximo do momento da interação. Mas esse caso é igualmente fácil e se resolver, porque tratando-se de uma emissão dura de um gluon, pode-se considerar que ela faz parte da colisão dura, e tratar desse caso como sendo de ordem superior em teoria de perturbação.

Outro tipo de interação inicial que pode ocorrer é entre quarks de hadrons diferentes. Se a interação for dura, ela ocorre no mesmo momento que a colisão em observação, porque estando a velocidades relativísticas os hadrons se contraem na direção do movimento, e os constituintes de cada um dos hadrons ficam separados dos constituintes do outro hadron por uma distância do tipo espaço, sendo portanto colisões independentes. Seria de se supor que houvesse problema numa troca de partons soft, porque nesse caso, o campo desse parton soft teria um alcance da ordem de $L \sim \frac{1}{q_{z}}$ onde $q_{z}$ é o momento do parton emitido, sendo $L$ grande o suficiente para que o campo dessa partícula entre em contato com outro hadron, propiciando uma troca de cor entre os dois hadrons. Porém, esse efeito pode ser incorporado na definição da função de distribuição, independentemente do processo, como foi mostrado nas ref. [57] e [58].

Há resultados recentes no entanto [49] mostrando que no caso específico de pomerons a seção de choque não pode ser fatorada, pois não é possível encontrar uma única distribuição de partons dentro do pomeron, que valha para todos os processos duros. Este resultado não afeta o presente trabalho, uma vez que não está sendo considerada a possibilidade de colisões duras do pomeron. 


\subsection{Geradores de Eventos}

São dois os programas de Monte Carlo geradores de eventos usados com maior freqüência na simulação de física para anéis de colisão hadrônicos: o ISAJET [63] e o PYTHIA [64]. O ideal teria sido fazer esse trabalho comparando justamente esses dois pacotes, porque os dois possuem já embutido o ferramental necessário para o estudo de colisões duras eletrofracas e fortes, incluindo as interações secundárias, constituídas de radiação eletromagnética e de gluons prevista pela QCD, fragmentação e hadronização, além de um extenso tratamento para as interações das partículas remanescentes dos feixes. O interesse maior na comparação dos dois programas reside no fato de apresentarem diferentes tratamentos às interações das partículas remanescentes dos feixes, após a colisão dura. O ISAJET analisa essas reações sob a ótica da unitariedade da matriz de espalhamento, implementada através da troca de pomerons de acordo com as regras de AGK [65]. Já o PYTHIA considera a interação principal (dura) e as demais independentes entre si, tendo como único elo o fato de compartilharem um total de energia e momento. Isso permite o uso de uma distribuição de probabilidade poissoniana para determinar o número de interações entre os partons dos protons iniciais que restaram após a colisão dura.

Porém, o ISAJET possui dois problemas que inviabilizam o seu uso num estudo em que justamente a distribuição final das partículas devidas aos processos secundários é importante:

1. Utiliza o Modelo Independente de Fragmentação, cujas primeiras idéias foram apresentadas nas ref. [66], e foi posteriormente desenvolvido por Field e Feynman [67]. Este modelo apresenta alguns problemas intrínsecos:

- O resultado do processo de fragmentação depende do referencial escolhido, não sendo invariante de Lorentz. Não é difícil contornar esse problema num caso em que serão produzidos dois jatos, mas no caso de muitos jatos não há como resolvê-lo.

- Outro problema é a presença de divergências colineares. Quarks e gluons produzem outros quarks e gluons de forma aproximadamente colinear, e as funções de fragmentação descrevem esse fato. Através desse processo obté-se a configuração final de partons a 
partir do processo duro (parton shower). No ISAJET, um parton sozinho, ou um grupo de partons sendo emitidos colinearmente produzem resultados muito diferentes, com multiplicidade maior no último caso.

2. O ISAJET apresenta uma multiplicidade final de partículas muito alta, incompatível com a esperada. Para processos duros, isso já era de esperar, devido ao problema de colinearidade apresentado anteriormente. Porém, o problema permanece nas colisões soft, indicando que talvez haja algum problema na implementação dos processos de minimum bias.

Há diversos outro modelos que utilizam descrições baseadas na unitariedade da matriz de espalhamento, e na troca de pomerons para as interações entre os hadrons, como o VENUS [68], e o QGSM [69] (Quark Gluon String Model). Porém, em lugar do ISAJET foi usado o DTUJET, por diversos motivos:

- A troca de pomerons seguindo um esquema de unitarização baseado nas regras de AGK [65] formam a base do modelo de interações múltiplas, que nesse trabalho deve ser comparada com a abordagem do PYTHIA.

- O modelo DTUJET está implementado na forma de um código Monte Carlo gerador de eventos, quesito essencial para ser usado nesse trabalho. Contém toda a física necessária para a descrição de uma colisão de hadrons, com exceção apenas das interações fracas (fato esse que precisou ser contornado) possuindo embutidas as subrotinas necessárias para executar fragmentação, hadronização, etc. O demais modelos têm uma forma mais rudimentar, e são dedicados a problemas específicos, como por exemplo a formação de quarks pesados, ou análise da difração dura, etc.

- Os resultados experimentais obtidos no CERN e no FERMILAB são explicados pelo DTUJET, e estão de acordo com as distribuições de partículas, energias, momentos, rapidez, etc. obtidas com ele. Por exemplo, a correlação entre o momento transversal médio dos hadrons produzidos e a multiplicidade de carga [70] e [80], o crescimento do patamar (plateau) de rapidez com a energia de colisão [80], o aumento 
do momento transversal médio com a energia de colisão [80], violação do scaling de Koba-Nielsen-Olsen (KNO) [81] da distribuição de multiplicidade, o efeito pedestal nos eventos de baixo $p_{T}$ subjacentes a jatos duros [80], as correlações forward-backward, as distribuições de rapidez e pseudorapidez e o crescimento dos momentos fatoriais são bem reproduzidos por esse modelo, além, é claro, de boa concordância com os dados obtidos para as seções de choque total, elástica e difrativa, conforme pode-se observar nas figuras da ref. [70], onde é feita a comparação.

O DTUJET não descreve os processos difrativos duros. Todavia esses processos não são incluídos no PYTHIA tampouco; o único programa que inclui esse tipo de processo, e que aliás é dedicado a ele é o POMPYT [71], e que no entanto é muito pouco divulgado.

\subsubsection{PYTHIA}

Denota-se por PYTHIA um pacote de dois programas, o próprio PYTHIA, e o JETSET [72]. Esse último possui as rotinas de fragmentação de jatos, decaimento de partículas, chuveiros de partons para o estado final e as rotinas específicas para análise do evento. Há duas opções para fragmentação, sendo que para esse trabalho foi escolhido o Modelo Lund de Fragmentação [73] (o outro modelo disponível é o Independente).

O PYTHIA possui as rotinas para gerar os processos duros e soft entre leptons, hadrons, gluons e fotons, incluindo todos os processos de interações múltiplas e difração, e para o tratamento dos chuveiros de partons do estado inicial.

Pelo fato dos dois programas, e toda a física neles utilizada, serem descritos em conjunto em um manual [74] extremamente completo e bem cuidado, esses tópicos não serão abordados nesse trabalho. No cap. (5) há uma discussão sobre a física diretamente envolvida com a formação de gaps de rapidez.

\subsubsection{DTUJET}

O DTUJET é um código Monte Carlo gerador de colisões proton-proton e proton-anti-proton. É baseado nas idéias do Dual Parton Model (DPM), que por sua vez é uma associação da Dual Topological Unitarization (DTU) com 
o modelo a partons. O DPM e o DTU são brevemente descritos a seguir.

Vários estudos de QCD não perturbativa usam abordagens onde se tomam grandes valores de $N_{f}$ (número de sabores) ou $N_{c}$ (número de cores), para criar artificialmente uma expansão topológica em que as interações são suprimidas por um fator $1 / N\left(N\right.$ pode ser $N_{f}$ ou $\left.N_{c}\right)$. Assim, interações representadas por diagramas topológicos complicados são suprimidas, sendo dominantes as que podem ser representadas por diagramas topológicos planares. A expansão mais usual é a proposta por t'Hooft [76], em que se toma $N_{c} \rightarrow \infty$, $\operatorname{com} \lambda=g^{2} N_{c}$ fixo, e com o número de sabores $N_{f}$ também fixo. A constante $g$ é a constante de acoplamento da QCD. Nesse caso, cada diagrama de Feynman pode ser representado por uma superfície orientada bidimensional, de forma que a expansão de Feynman pode ser reagrupada como uma soma de superfícies de determinadas topologias, e o peso de cada termo dessa soma é aumentado de um fator $N_{c}^{-2}$ quando o numero de handles (alças) da superfície aumenta de uma unidade. Desta forma, as amplitudes são dominadas por diagramas planares.

O Dual Topological Unitarization(DTU) utiliza uma expansão topológica alternativa proposta por Veneziano [77] que é mais adequada para a descrição da fenomenologia quando se está interessado em colisões a altas energias (em que a produção de partículas por processos inelásticos domina), no qual se adota um limite onde tanto $N_{f}$ quanto $N_{c}$ são tomados grandes, com a restrição de que a razão $N_{f} / N_{c}$ permanece fixa (além de $\lambda$ ). Essa expansão estabelece os vínculos da unitariedade e da dualidade.

O DPM (Dual Parton Model) foi introduzido em Orsay em 1979, e incorpora idéias do DTU e do modelo a partons [75], proporcionando uma estrutura fenomenológica para o conceito de pomeron. Associa uma seção de choque a cada diagrama proveniente da expansão baseada no DTU. Esses diagramas envolvem trocas de múltiplos pomerons no canal $t$ e têm uma correspondência direta com os diagramas usados na Teoria de Campos de Reggeons [45] e [65] (RFT, Reggeon Field Theory).

Possui duas componentes principais, uma soft, descrita pelo pomeron supercrítico, e outra hard, descrita pela QCD perturbativa. O conceito de cor não aparece explicitamente no modelo (a não ser é claro nos fatores que surgem no cálculo das amplitudes de interações de quarks e gluons), mas sua presença está implícita no conceito de strings a que ele faz recurso.

A amplitude elástica, e portanto a seção de choque total, é calculada a partir da teoria de trocas múltiplas de pomerons desenvolvida por Gribov. 

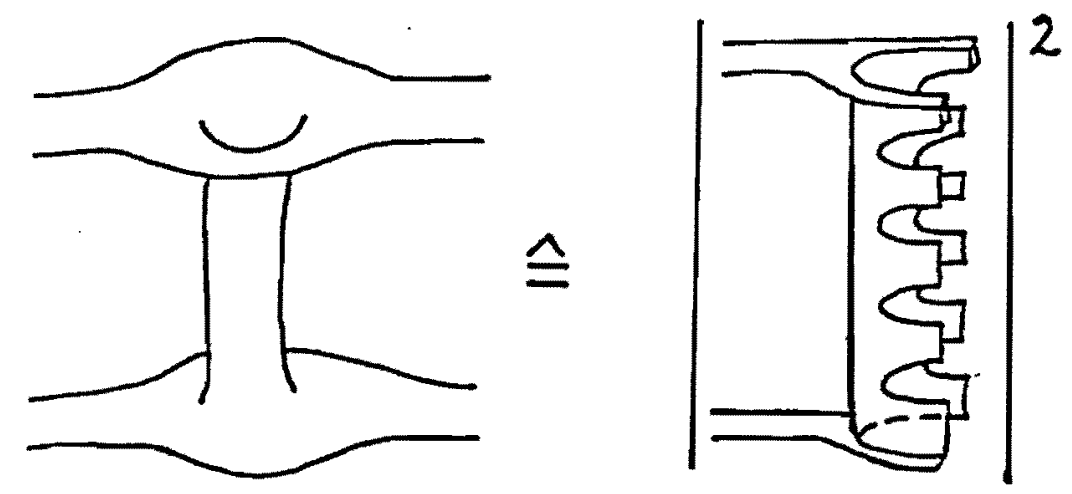

Figura 4.5: Troca de um pomeron e sua topologia cilíndrica.

As amplitudes elásticas são representadas na forma eikonal, com a eikonal sendo a transformada de Fourier do propagador do pomeron. A contribuição dominante numa colisão elástica a altas energias é a troca de um pomeron, que tem a topologia de um cilindro (uma superfície planar bidimensional), conforme pode ser visto na fig. (4.5), e é constituído por uma rede de gluons e loops fechados de quarks.

Processos inelásticos são relacionados a pomerons cortados, e o peso de cada classe de processo inelástico é calculado a partir de seções de choque topológicas $\sigma_{m}$, que correspondem a $m$ pomerons cortados.

Quando um pomeron é cortado, devido à sua forma, dá origem a dois strings (ou duas cadeias) (ver fig. (4.6)) possuindo quarks ou gluons em suas extremidades; os strings posteriormente se transformarão em hadrons (hadronização). Desta forma, se $k$ pomerons forem cortados, a interação possuirá $2 k$ strings, sendo que cada uma delas terá suas extremidades acopladas a quarks de valência ou quarks ou gluons do mar, (as extremidades de uma cadeia sempre se acoplam a constituintes do hadron). É possível haver a formação de somente uma cadeia, no caso da troca de um reggeon, ou seja, quando há troca de sabor, pois o diagrama do reggeon é o mostrado na fig. (4.7), e após um corte, forma-se apenas um string. Porém, esse tipo de diagrama é suprimido com $s^{-1}[78]$. 


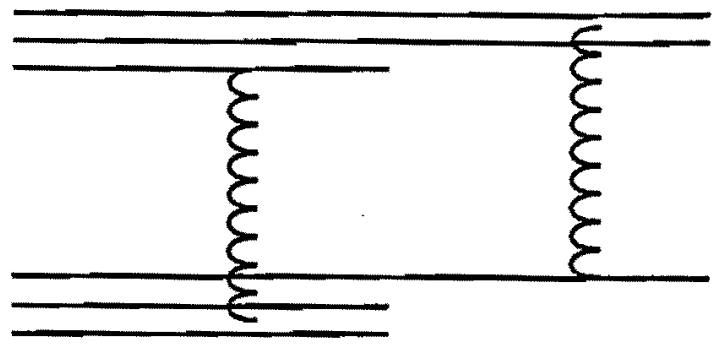

Figura 4.6: Diagrama com duas cadeias, resultante do corte de um pomeron.

Neste ponto, é importante ressaltar alguns aspectos que serão cruciais na análise da probabilidade de formação do gap de rapidez:

- Em primeira ordem, o proton é constituído de um quark de valência e um diquark de valência, e a interação entre os hadrons dá origem a duas cadeias que se estendem entre esses constituintes, conforme a fig. (4.8a). O acoplamento das cadeias é feito entre partons ou diquarks de um dos hadrons com partons ou diquarks do outro. Nunca ocorre formação de cadeia entre dois partons de um mesmo hadron.

- A troca de um pomeron duro na terminologia do DTUJET equivale completamente a uma colisão dura parton-parton na descrição do PYTHIA.

- Sempre que houver troca de um pomeron duro, há também troca de um pomeron soft (4.9a). A função desse pomeron soft é fornecer os strings que efetuarão as trocas de cor necessárias à neutralização das partículas remanescentes dos feixes iniciais. Esses strings, ou cadeias, serão posteriormente fragmentados. Desta formá, a fragmentação da parte remanescente do feixe inicial, por ser também uma interação entre os partons dos hadrons incidentes, está vinculada, assim como todos os outros processos que podem ocorrer durante a interação dos dois hadrons, ao esquema de unitarização utilizado. 


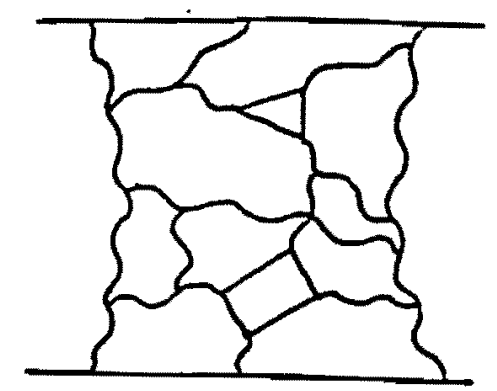

Figura 4.7: Diagrama mostrando a troca de um Reggeon.

$\mathrm{O}$ que distingue os vários modelos baseados no Dual Parton Model é a forma de produção dos strings (ou seja, a forma como a cor é trocada). $\mathrm{O}$ DTUJET tem uma opção que possibilita o uso da fragmentação de Lund, implementada no código JETSET, e que é a mesma utilizada no PYTHIA. Portanto o DTUJET trabalha até o momento de produzir os strings, e em seguida passa a atuar o JETSET, através da fragmentação e posterior hadronização, produzindo hadrons, que por sua vez decaem se forem instáveis.

\section{Cálculo das seções de choque no DPM}

O modelo tem uma componente soft, representada pelo pomeron supercrítico ${ }^{1}$ e outra dura (hard), descrita pela QCD perturbativa (pomeron duro). As ref. $[70,78,80]$, apresentam a descrição detalhada do modelo, que será apenas delineado aqui.

A troca de um pomeron corresponde a uma seção de choque soft pura, $\sigma_{s}$, parametrizada por :

$$
\sigma_{s}=a s^{\alpha(0)-1}
$$

sendo $a=g^{2}=37.8 \mathrm{mb}$ o acoplamento proton-pomeron e $\alpha(0)=1.08 \mathrm{a}$ intercecção da trajetória do pomeron supercrítico. A correspondente trajetória do pomeron é dada por: $\alpha(t)=\alpha(0)+\alpha^{\prime} t$. A expressão (4.6) viola o

\footnotetext{
${ }^{1}$ Entende-se por pomeron supercrítico, um pomeron cuja trajetória tem uma intersecção maior que 1 para levar em conta o pequeno aumento com a energia observado na seção de choque total.
} 

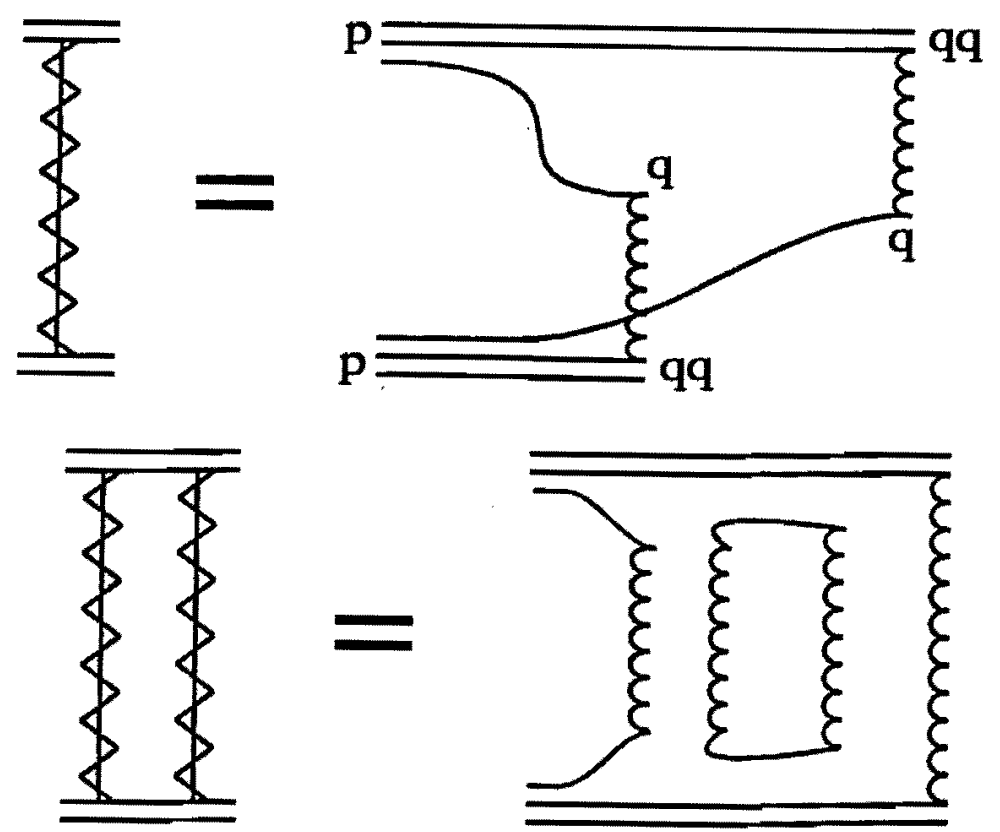

Figura 4.8: Diagramas para a troca de pomerons soft:(a)um pomeron (duas cadeias);(b)dois pomerons (quatro cadeias). Todos os pomerons estão representados cortados.

limite de unitariedade a altas energias, tornando-se necessária a inclusão de mais pomerons para restabelecer a unitariedade.

A fig. (4.8a) mostra a troca de um pomeron supercrítico (soft), enquanto a fig. (4.8b) mostra um diagrama de ordem superior, envolvendo a troca de dois pomerons.

A componente dura é descrita pela seção de choque da QCD perturbativa, que em ordem mais baixa é dada por [82]:

$$
\sigma_{h}=K \sum_{a, b} \iiint d x_{a} d x_{b} d \hat{t} x_{a} f_{a / A}\left(x_{a}, Q^{2}\right) x_{b} f_{b / B}\left(x_{b}, Q^{2}\right) \frac{1}{x_{a} x_{b}} \pi M^{2} \frac{\alpha^{2}\left(Q^{2}\right)}{\hat{s}^{2}},
$$

onde:

- $f_{i / I}\left(x_{i / I}, Q^{2}\right)$ é a distribuição de partons $i$ no hadron $I$. 

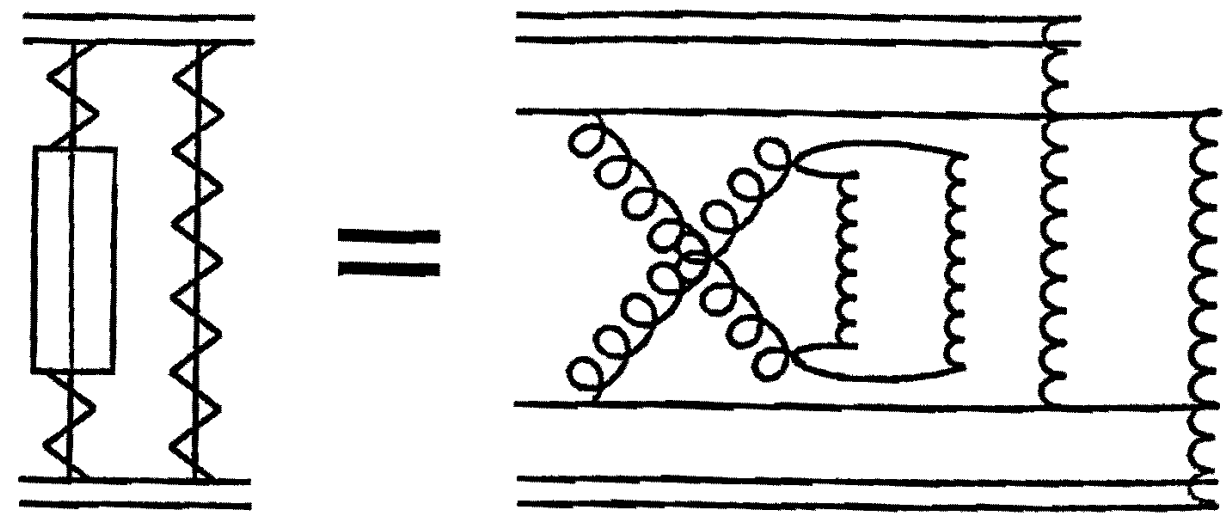

Figura 4.9: Diagrama correspondente à troca de um pomeron soft e um duro.

- $M=M_{a, b \rightarrow c . d}$ é o elemento de matriz para o espalhamento duro partonparton $a+b \rightarrow c+d$.

- $\alpha_{s}\left(Q^{2}\right)$ é a constante de acoplamento forte.

- $K$ é um fator que substitui a inclusão de correções de ordens superiores ao nível de árvore.

A fig. (4.9a) mostra a troca de um pomeron duro e outro soft, e a fig. (4.9b) apresenta a troca de um pomeron soft e dois duros. As interações duras são independentes umas das outras, no sentido de que não há interferência entre os pomerons trocados. $O$ número de jatos duros e minijatos em um evento é proporcional ao número de espalhamentos duros, e portanto a multiplicidade média de jatos deve aumentar com a energia.

A seção de choque da eq. (4.7) apresenta forte dependência com o corte inferior de momento transversal $p_{\perp, m i n}$, introduzido para garantir que a teoria de perturbaçōes permaneça válida. $\mathrm{O}$ valor usado como default no programa é $2.0 \mathrm{GeV}$.

A seção de choque dura é proporcional a uma potência de $s$, e também viola a unitariedade, e a altas energias torna-se maior que a seção de choque total, que não pode crescer mais rapidamente que $\ln s^{2}$. 
Além do pomeron supercrítico e do pomeron duro, há duas outras componentes que dão conta das interações pomeron-pomeron, cujo acoplamento é muito menor que $g$. Essas interaçōes dão origem a processos difrativos, e não serão discutidas neste trabalho. Uma discussão sobre esses aspectos do modelo pode ser encontrada nas referências já citadas $[80,70,78]$.

Processos Soft (s), duros (h) e as interações pomeron-pomeron (si) são tratados simultâneamente com um esquema de unitarização eikonal que usa a representação de parâmetro de impacto:

$$
\chi_{i}(B, s)=\frac{\sigma_{i}(s)}{8 \pi b_{i}} \exp \left(\frac{-B^{2}}{4 b_{i}}\right), \quad i=s, h, s i
$$

com a normalização:

$$
\int 2 \chi_{i}(B, s) d^{2} B=\sigma_{i}
$$

sendo $b_{i}$ os parâmetros de impacto. $b_{h}$ é independente da energia, $b_{s}=b_{s i}=$ $b+\alpha^{\prime} \log s$.

A seção de choque exclusiva para $l_{c}$ soft pomerons cortados, $m_{c}$ pomerons duros cortados, é dada por:

$$
\sigma\left(l_{c}, m_{c}, n_{c}, p_{c}, B, s\right)=\frac{\left(2 \chi_{s}\right)^{l_{c}}}{l_{c} !} \frac{\left(2 \chi_{h}\right)^{m_{c}}}{m_{c} !} F_{s i} \exp [-2 \chi(B, s)],
$$

onde $F_{s i}$ é um fator que inclui as interaçöes pomeron-pomeron, e

$$
\chi(B, s)=\chi_{s}(B, s)+\chi_{h}(B, s)-\chi_{s i}(B, s)
$$

As seções de choque total e elástica são dadas por:

$$
\begin{gathered}
\sigma_{t o t}=4 \pi \int_{0}^{\infty} B d B(1-\exp [\chi(B, s)]), \mathrm{e} . \\
\sigma_{e l}(B, s)=\frac{1}{4}\left[\sigma_{t o t}(B, s)\right]^{2} .
\end{gathered}
$$




\section{Capítulo 5}

\section{Gap de Rapidez}

Uma região de rapidez que não contenha nenhum hadron é chamada convencionalmente de gap de rapidez. Sua existência em física relacionada a espalhamentos elásticos e difrativos, com pequeno momento transferido, é bastante conhecida, ocorrendo provavelmente em conseqüência da troca de pomerons.

A possibilidade de existência de um gap de rapidez em processos de formação de um higgs foi sugerida em 1986 [84] por Dokshitzer, Khoze e Troyan, em virtude da necessidade de se distinguir o modo pelo qual um boson de Higgs se formou. Essa distinção é importante entre outros motivos, porque a melhor determinação possível dos vértices $W W H$ e $Z Z H$ é de grande interesse na confirmação do Modelo Padrão, ou então, no direcionamento da escolha de uma extensão para esse modelo.

Há um outro bom motivo para se estudar a formação de gaps de rapidez. Um processo do tipo $V V \rightarrow V V$, onde $V$ designa um boson vetorial com massa, leva a estados finais completamente diferentes conforme sejam analisados sob a luz de modelos como o Dual Parton Model, ou de modelos de fragmentação como o Lund Model. No primeiro caso não se espera a formação de um gap de rapidez, e no segundo, sim. Se o gap for bem visível, será possivel escolher entre um modelo e outro.

Considerando-se a massa do quark top como $174 \mathrm{GeV}$, um higgs com massa maior que $m_{W}$ deve ser produzido principalmente por fusão de bosons vetoriais e por fusão de gluons, sendo essa última possibilidade mais provável qualquer que seja a massa do higgs (sec. 3.3). O fluxo de cor nesses dois processos é bastante diferente, e como conseqüência, pode ou não surgir o 
gap de rapidez.

Numa fusão de bosons W ou Z (fig. (5.1)) não há transferência de cor entre os dois feixes que participam da colisão, pois um quark de cada um dos protons emite o boson W (ou Z), que é singleto de cor, permanecendo o estado de cor do proton inicial inalterado. Esses bosons se aniquilam, produzindo o higgs, que é um singleto também. Segundo a aproximação de W efetivo [85] os bosons vetoriais que entram nesse processo são emitidos por quarks que possuem grande energia e adquirem um momento transversal da ordem de $M_{W}$ no momento da emissão, estando por isso localizados em regióes de grande rapidez. Portanto, a princípio, pode-se considerar como assinatura para esse processo a presença de dois tagging jets com grande energia, grande rapidez, e a existência de poucos, ou nenhum hadron na região de rapidez intermediária.

Na fusão de gluons (fig. (5.2)) o proton emite um octeto de cor, e portanto o que resta dele, o feixe remanescente é um anti-octeto de cor. Os dois gluons emitidos se aniquilam, formando o higgs, e os dois feixes que restaram precisam interagir de alguma forma para voltar ao estado de singleto; essa interação, que ocorre com uma troca de cor entre os feixes remanescentes, leva ao processo de hadronização preenchendo com hadrons a região entre os dois feixes.

É possível, além disso, que os gluons interagentes emitam outros gluons na forma de radiação, o que faz com que seja ainda maior o número de hadrons na região central do detetor (pequenos valores de rapidez). O mesmo não ocorre com a fusão de bosons vetoriais, pois eles não emitem gluons.

Um lego plot representa o plano pseudo-rapidez $\times$ angulo azimutal de um evento; na fig. (5.3) está mostrado um evento fictício de fusão de bosons $W$ para esclarecer sua forma de apresentação. Os jatos mostrados na figura são os tagging jets que provêm da hadronização dos quarks que emitiram os W's. Considera-se o jato como possuindo um raio de 0.7 unidades nesse plano pseudo-rapidez $\times$ ângulo azimutal, e traça-se uma tangente à borda do jato conforme está mostrado na figura citada. Essa região interna às duas tangentes pode ou não ser preenchida por hadrons, caracterizando ou não a existência do gap. As fig. (5.4) e (5.5) apresentam respectivamente para uma fusão de W's e uma fusão de gluons, um lego plot e o correspondente histograma bidimensional (esses dois eventos foram produzidos com o PYTHIA, sendo utilizados os parâmetros descritos no cap. (6).

No entanto, diversos problemas surgem quando se tenta utilizar essa assi- 


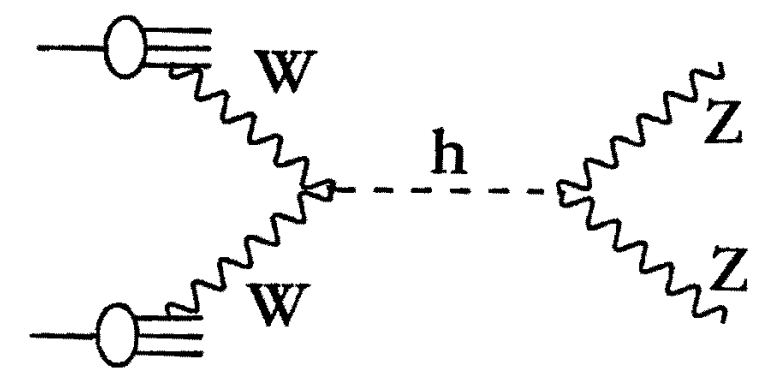

Figura 5.1: Diagrama de Feynman para o processo $p p \rightarrow W W X \rightarrow h X \rightarrow$ $Z Z X$

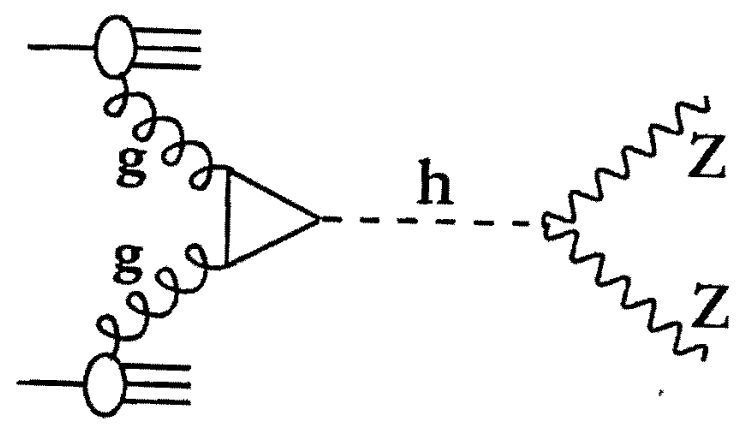

Figura 5.2: Diagrama de Feynman para o processo $p p \rightarrow g g X \rightarrow h X \rightarrow$ $Z Z X$ 


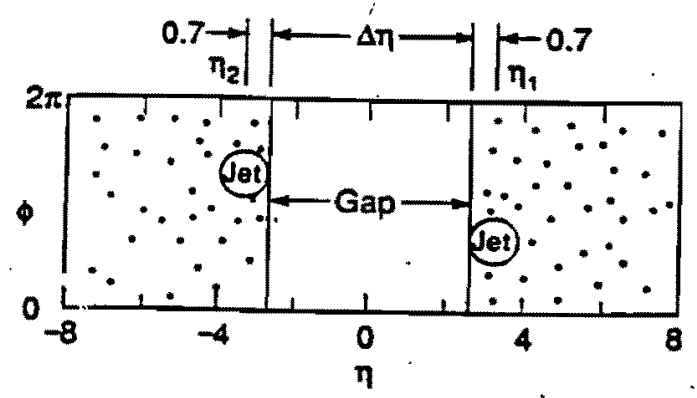

Figura 5.3: Lego plot para um evento ficticio

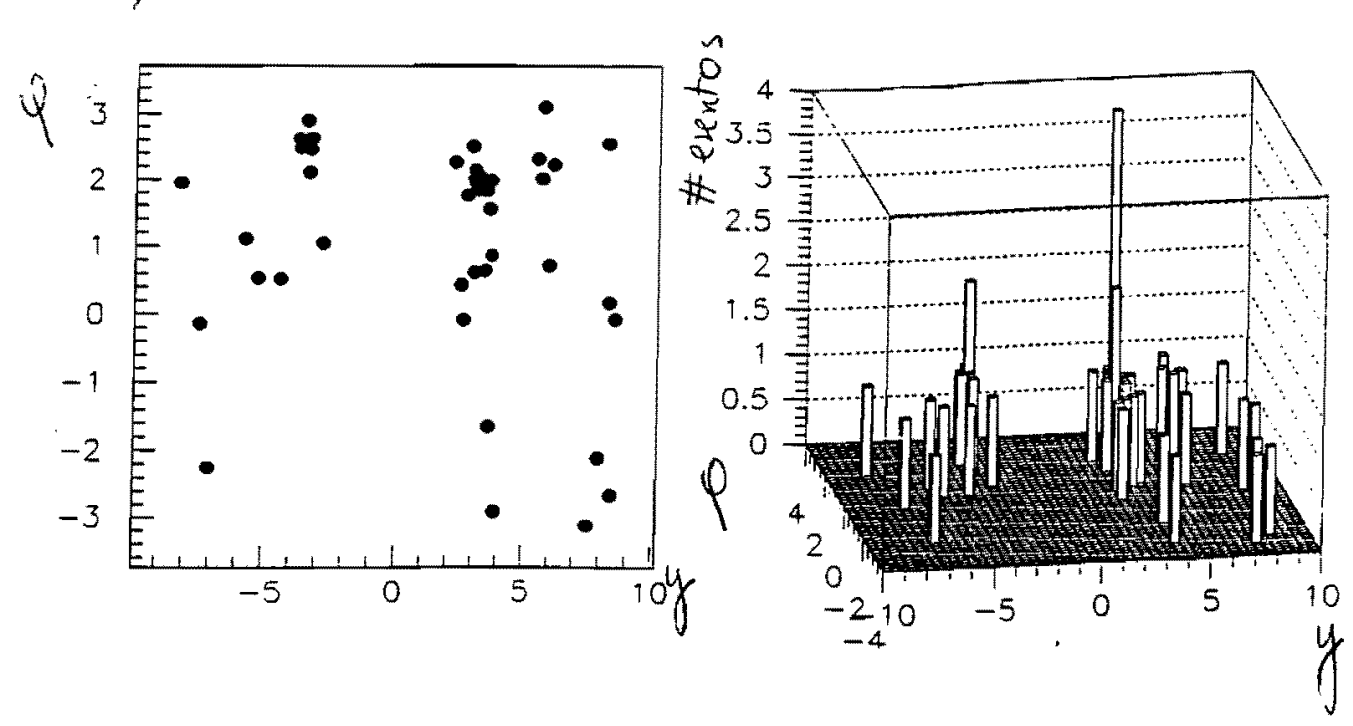

Figura 5.4: Lego plot e correspondente histograma bidimensional para uma fusão de W's. 

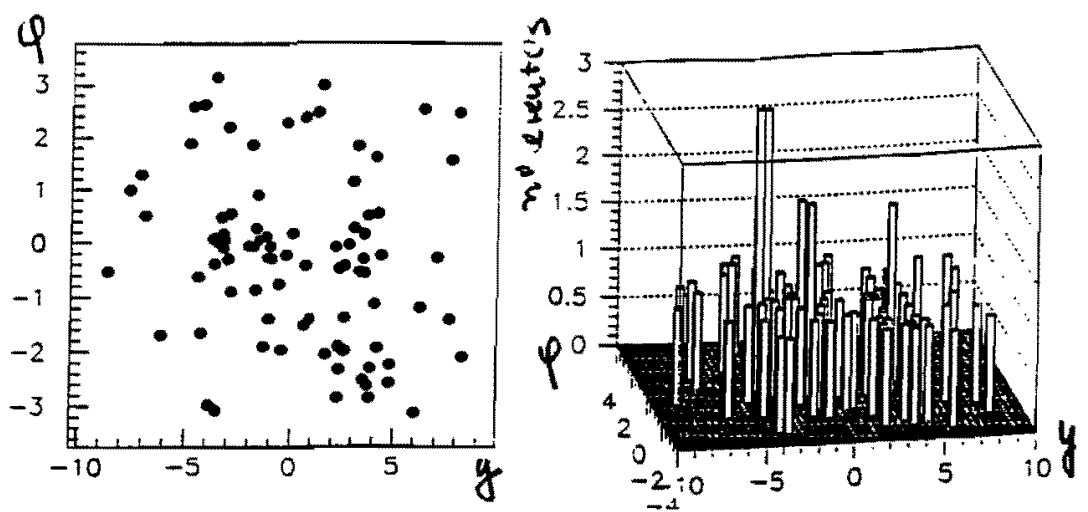

Figura 5.5: Lego plot e correspondente histograma bidimensional para uma fusão de gluons.

natura para separar a fusão de W's da fusão de gluons. Será visto que ela é de muito difícil deteção, além de poder ser imitada por vários outros processos, e o seu estudo na verdade esbarra em aspectos não completamente estabelecidos das interações como a fragmentação e no talvez mais obscuro ponto das colisões badrônicas, que é o das interações múltiplas, que ocorre quando há mais que uma reação partônica na colisão de dois protons.

A impossibilidade de se usar QCD perturbativa na análise de eventos de minimum bias ${ }^{1}$ impede um conbecimento satisfatório desse tipo de processo, embora não haja dúvida de que a QCD seja a teoria que descreve corretamente essas interações. Há diversos modelos que tentam descrever essa física de interações hadrônicas, sendo que muitos deles em boa concordância com os dados experimentais atuais, mas partindo de princípios não somente diferentes, como antagônicos, e com prediçōes diferentes para energias mais altas que as testadas até o momento. Esse tipo de evento é o mais freqüente nas interações hadrônicas, sendo que os processos duros, passíveis de descrição perturbativa são extremamente raros.

Quando dois protons, que são compostos de diversos partons, colidem, é possível que haja mais que uma colisão partônica, ocórrendo as chamadas interações múltiplas. Esse ponto envolve também muita discordância, por exemplo a respeito da distribuição de probabilidade para o número de possiveis reações numa colisão de protons.

\footnotetext{
${ }^{1}$ processos elásticos, simples e duplamente difrativos e de pequeno momento transversal trocado.
} 
Outra dificuldade a ser mencionada, é a possibilidade de ocorrência de pile up (evento em que há mais do que uma colisão de protons num mesmo bunch crossing), em virtude da alta luminosidade dos aceleradores. Esse fato aumenta bastante o número de partículas produzidas na região central do detetor, prejudicando assim a observação de um possível evento com gap.

Em diversos momentos na análise haverá necessidade de se adotar um modelo, ou comparar os resultados apresentados por dois ou mais modelos, e para isso serão utilizados os códigos já apresentados, DTUJET e PYTHIA.

\subsection{Dificuldades na Detecção do Gap}

\subsubsection{Fragmentação}

Um primeiro problema a se considerar é a possibilidade de que algumas das partículas resultantes da fragmentação dos feixes remanescentes ou do quark espectador acabem preenchendo o gap, principalmente quando um dos jatos formados a partir do quark que emitiu o boson vetorial tem rapidez muito grande pois então muitas partículas do feixe remanescente estarão próximas dele no espaço de rapidez levando em alguns casos à contaminação do gap. Esse problema pode ser parcialmente resolvido modificando-se a definição de gap: em vez de ser a região interior às duas retas tangentes mostradas na fig. (5.3), considera-se um $\Delta y$ fixo, centrado em zero. Por exemplo, procurase saber quantas partículas de um determinado evento possuem rapidez entre $-2.5 \mathrm{e}+2.5$, independentemente da posição dos tagging jets.

Um olhar mais atento leva à conclusão de que esse problema está ligado também a uma outra questão mais complexa, que é a falta de um modelo único para a fragmentaçăo. Para a fusão de gluons (fig. (5.7)) o PYTHIA e o DTUJET apresentam soluçôes diferentes, mas que levam ao mesmo estado final: se o quark que emitiu o gluon possuía cor $\underline{a}$, após a emissão passará a ter outra cor $\underline{b}$; para que os gluons emitidos possam se aniquilar formando um singleto, o quark emissor do outro proton deve ter cor inicial $\underline{b}$, passando após a emissão para $\underline{a}$. Os feixes remanescentes ficarão respectivamente com cores $\bar{a}(\equiv b c)$ e $\bar{b}(\equiv a c)$. Para que esses feixes possam formar hadrons brancos, é preciso haver uma troca de cores entre o quark emissor de cada um dos protons e o diquark remanescente do outro. No PYTHIA isso é feito através de dois strings ligando esses dois pares de quark-diquark, que em seguida se 

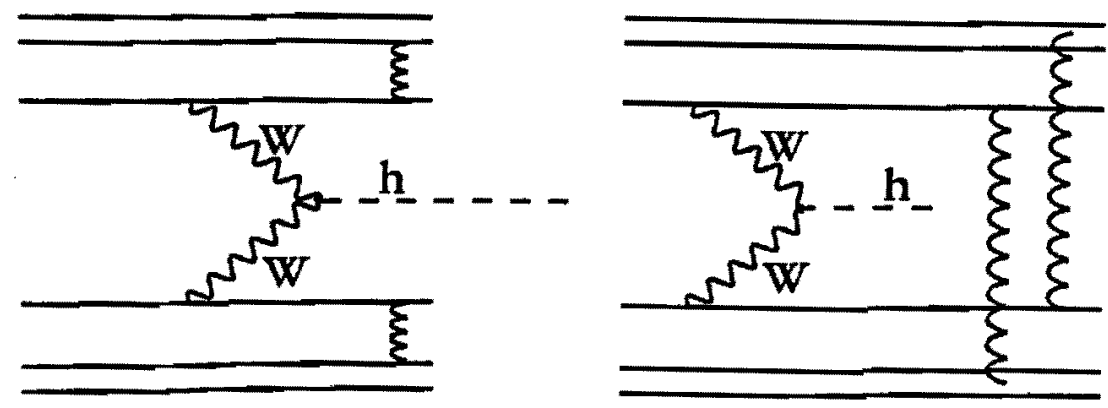

Figura 5.6: Formação de strings numa fusão de W's: a) segundo o modelo LUND (PYTHIA); b) segundo o DTUJET.

fragmentam, dando origem aos hadrons finais. No DTUJET, essa troca de cor é feita através de um pomeron cortado. Apesar do pomeron possuir os números quânticos do vácuo, e portanto não transportar cor, ele é composto por uma rede de gluons e loops fechados de quarks, em que a cor circula. No momento em que ele é cortado, os quarks e gluons que estavam nas linhas dos cortes formam cadeias de hadrons, e a cor flui, subindo em uma cadeia, e descendo na outra, não havendo portanto, troca líquida de cor, mas deixando todos os jatos como singletos.

Já no caso da fusão de W's (fig. (5.6), essas soluções diferentes levam a estados finais completamente diversos. Um dos quarks emissores tem cor $\underline{a}$, e portando o respectivo feixe remanescente possue cor $\bar{a}$, o mesmo ocorrendo com o outro proton para alguma cor $\underline{b}$, que pode ou não ser igual a $\underline{a}$. No modelo LUND, quando um dos quarks e o feixe remanescente do mesmo proton iniciam o afastamento, por formarem um par cor-anticor;imediatamente uma string se forma entre eles, que posteriormente se fragmenta; observe que essa string fica somente entre o quark emissor e o feixe remanescente, o mesmo ocorrendo para o outro proton [86]. Por esse motivo, não há formação de hadrons na região central de rapidez. No DTUJET, por outro lado, a troca de pomeron ocorre da mesma maneira que na fusão de gluons, porque as cadeias 

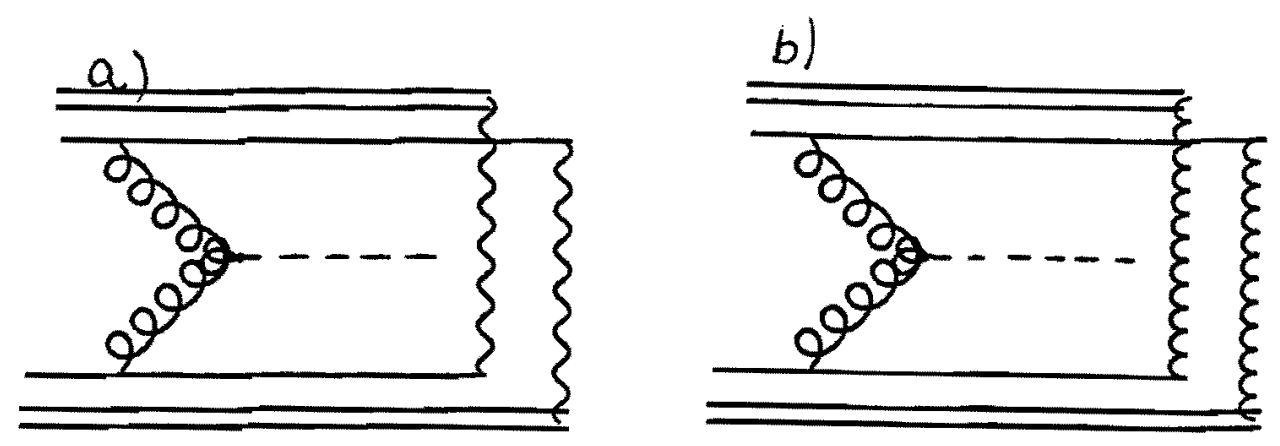

Figura 5.7: Formação de strings numa fusão de gluons: a) segundo o modelo LUND (PYTHIA); b) segundo o DTUJET.

do pomeron sempre se formam entre o quark de um proton e o diquark do outro, dando origem portanto a hadrons na região central de rapidez. Ainda assim, mesmo quando é usado o PYTHIA como gerador de eventos, pode ser que o gap seja preenchido devido ao motivo anteriormente citado.

\subsubsection{Interações Múltiplas}

Sendo o proton composto de vários partons, é possível que numa interação entre dois protons haja mais do que uma colisão partônica. As interações desse tipo serão chamadas Interações Múltiplas.

Não há um modelo completamente satisfatório para descrição das interações múltiplas, pelo fato delas envolverem interações de QCD com pequeno momento transversal trocado, e portanto não serem passíveis de descrição por teoria de perturbações. O DTUJET e o PYTHIA tratam esse assunto de forma completamente diferente.

O PYTHIA é um programa elaborado para descrever interações duras. Todos seus outros elementos, como radiação eletromagnética e de gluons, fragmentação, interação das partículas remanescentes dos feixes, etc. são introduzidos no sentido de aprimorar a descrição do processo central, que é 
a colisão dura. Da mesma forma, a inclusão da possibilidade de interações múltiplas no PYTHIA tem a intençăo de auxiliar a análise do processo duro, permitindo avaliar o quanto um sinal do processo principal é enfraquecido (ou talvez perdido) pelo fato de ocorrer mais do que uma reação no mesmo evento.

Já o DTUJET tem como interesse primordial a descrição das colisões hadrônicas como um todo. Sob esse ponto de vista, as interações duras perdem parte de seu interesse, pois são eventos raros comparativamente com os processos difrativos e todos os que ocorrem com pequena troca de momento transversal, tendo sido introduzidas no programa somente recentemente, como um refinamento. Por esse motivo, a abordagem é completamente diferente. Há apenas um ponto em comum, apresentado a seguir.

A seção de choque diferencial da QCD para processos $2 \rightarrow 2$ duros é dada por:

$$
\frac{d \sigma_{h}}{d p_{t}^{2}}=\sum_{i, j, k} \int d x_{1} \int d x_{2} \int d \hat{t} f_{i}\left(x_{1}, Q^{2}\right) f_{2}\left(x_{2}, Q^{2}\right) \frac{d \hat{\sigma}_{i j}^{k}}{d \hat{t}} \delta\left(p_{t}^{2}-\frac{\hat{t} \hat{u}}{\hat{s}}\right),
$$

onde $\sigma_{i j}^{k}$ indica a seção de choque para o subprocesso $k$ envolvendo os partons $i$ e $j, f_{i}\left(x_{1}, Q^{2}\right)$ representa a funç̧ão de distribuição de partons $i$ dentro do proton, com fração de momento entre $x_{1}$ e $x_{1}+d x_{1}\left(f_{j}\left(x_{2}, Q^{2}\right)\right.$ tem significado equivalente para o parton $j$ ).

Assume-se que a escala usada na violação de scaling, $Q^{2}$, seja da ordem de $p_{\perp}^{2}$ do espalhamento.

A seção de choque para espalhamento duro fica:

$$
\sigma_{h}\left(p_{t_{\min }}\right)=\int_{p_{t_{\min }}^{2}}^{s / 4} \frac{d \sigma}{d p_{t}^{2}} d p_{t}^{2} .
$$

Como a seção de choque diferencial diverge $\frac{d \sigma}{d p_{\perp}^{2}} \sim \frac{1}{p_{\perp}^{4}}, \sigma_{h}$ também é divergente para $p_{t_{\min }} \rightarrow 0$.

$\sigma_{h}$ fornece a seção de choque partônica, ou seja, a probabilidade de que ocorram interações entre os partons do hadron, em função de $p_{t}$. Já a eq. (4.1) permite obter $\sigma_{n d}$, que é a seção de choque para processos não difrativos, ou seja, a probabilidade de que dois hadrons interajam, sem produzir processos difrativos. Portanto, a razão $\bar{n}_{h}=\sigma_{d u r a} / \sigma_{n d}$ dá o número médio de interações por evento. 


\section{PYTHIA}

A implementação das interações múltiplas no PYTHIA será apresentada de forma extremamente esquemática aqui. $O$ tratamento completo é apresentado na ref. [87].

Partindo-se do pressuposto de que essas interações sejam independentes (exceto pelo fato de dividirem entre si a energia total disponivel no evento), o número de interações em um evento é fornecido por uma distribuição de Poisson com média $n_{h}$.

Há uma versão mais sofisticada desse modelo de interações múltiplas, apresentada na mesma referência, que leva em consideração o fato do hadron ser extenso, e com uma distribuição não homogênea de partons em seu interior. Essa versão não foi a utilizada no trabalho, e portanto não será apresentada aqui.

Portanto o número de espalhamentos por evento é distribuído de acordo com uma curva de Poisson com média $\bar{n}$.

Considerando-se $x_{t}=2 p_{t} / E_{c m}$, pode-se definir a probabilidade de que haja uma interação parton-parton com $x_{t}=x_{t_{i}}$ como:

$$
f\left(x_{t}\right)=\frac{1}{\sigma_{n d}(s)} \frac{d \sigma}{d x_{t}}
$$

com $d \sigma / d x_{t}$ obtida como na eq. (5.1).

A probabilidade de que haja uma interação com $x_{t} \geq x_{t_{i}}$ fica dada por:

$$
F\left(x_{t_{i}}\right)=\int_{x_{t_{i}}}^{1} f\left(x_{t_{i}}\right) d x_{t_{i}} .
$$

Para levar em conta a energia já utilizada, para o i-ésimo espalhamento, toma-se a distribuição de partons para:

$$
x_{i}^{\prime}=\frac{x_{i}}{\sum_{j=1}^{i-1} x_{j}}
$$

Em uma fração $\exp \left(-F\left(x_{\min }\right)\right)$ dos eventos gerados, não há nenhum espalhamento duro acima de $x_{t_{\min }}$; são os espalhamentos soft. Nesses casos, os espalhamentos são considerados com igual probabilidade como sendo:

- espalhamento do tipo $g g \rightarrow g g$, com os gluons num estado singleto de cor; 
- espalhamento do tipo $g g \rightarrow g g$, com cada um dos gluons conectado a um string já existentes.

- espalhamento $g g \rightarrow q \bar{q}$, com o par final em um estado singleto de cor.

\section{DTUJET}

No DTUJET o número médio de interações duras é obtido da mesma forma que para o PYTHIA. Porém além disso, o número de espalhamentos soft é escolhido dessa maneira também (e portanto de maneira diferente do PYTHIA), sendo dado por: $n_{s}=\sigma_{s} / \sigma_{n d}$, sendo $\sigma_{s}$ dado pela eq. (4.6).

O peso para um evento com $n_{h}$ colisões duras e $n_{s}$ colisões soft é dado por um esquema de unitarização que é uma generalização das regras de corte de AGK [65]. Aqui reside o ponto em que o DTUJET é substancialmente diferente do PYTHIA. Ele parte de uma propriedade geral da matriz de espalhamento $S$, que é a unitariedade, e a partir dela desenvolve toda a teoria de interações.

\subsubsection{Pile Up}

Devido à alta luminosidade com que se trabalha nos anéis de colisão, é grande a probabilidade de que ocorra mais do que uma interação no mesmo bunch crossing. Os eventos em que ocorre esse efeito são chamados de pile up events.

A ocorrência de pile up quando é produzido um evento com gap de rapidez deve prejudicar sua assinatura, pois as outras interações muitas vezes produzirão partículas na região central, fazendo com que o gap seja contaminado.

O DTUJET não possui geração de eventos pile up, e a forma como é implementado no PYTHIA apresenta problemas técnicos de computação ${ }^{2}$. Por esse motivo, não será possível comparar os dois programas sob esse aspecto.

Supondo-se que cada uma das colisões seja independente, ou seja, que não haja interferência entre as partículas de diferentes colisões, pode-se usar uma distribuição de Poisson para obter a probabilidade de que um evento ocorra

\footnotetext{
${ }^{2} \mathrm{O}$ programa reserva uma determinada quantidade de memória para guardar informações sobre as partículas finais de cada evento, e esse espaço não é suficiente no caso de pile $u p$, devido ao grande número de partículas presentes no estado final.
} 
com determinado número de colisões proton-proton. O número médio de colisões proton-proton que ocorrem num mesmo bunch crossing é dado por:

$$
\bar{N}=\sigma \cdot \mathcal{L} \cdot \Delta t_{c o l}
$$

sendo $\sigma$ a seção de choque dos processos que estão sendo considerados, $\mathcal{L}$ a luminosidade do acelerador e $\Delta t_{c o l}$ é o intervalo de tempo entre dois bunch crossings.

No caso do LHC, tem-se:

- $\sigma_{p p} \sim 80$ mbarn.

- $\mathcal{L} \sim 100$ fbarn $^{-1}$ ano $^{-1}=10^{7} \mathrm{mbarn}^{-1} \mathrm{~s}^{-1}$.

- $\Delta t_{c o l}=0.025 \mu s=2.5 \times 10^{-8}$.

Portanto:

$$
\bar{N} \sim 80 \cdot 10^{7} \cdot 2.5 \times 10^{-8}=20 \text { colisões por bunch crossing. }
$$

Conclui-se que o número médio de eventos em um mesmo bunch crossing é 20. Todavia, neste trabalho foram usados os valores 8 e 12 como números médios de eventos por bunch [88].

\subsection{Probabilidade de Sobrevivência do Gap}

Devido às dificuldades mencionadas na detecção do gap de rapidez, tornase necessário de alguma forma quantificar a probabilidade de que ele seja realmente encontrado para analisar a viabilidade de sua utilização em experimentos. Björken [89] propôs chamar a fração de eventos que contém efetivamente o gap dentre todos os eventos possíveis geradores de um gap de 'probabilidade de sobrevivência do gap de rapidez', que sérá representada por $<|S|^{2}>$. A próxima seção mostra o cálculo dessa probabilidade de acordo com o modelo eikonal [89]. 


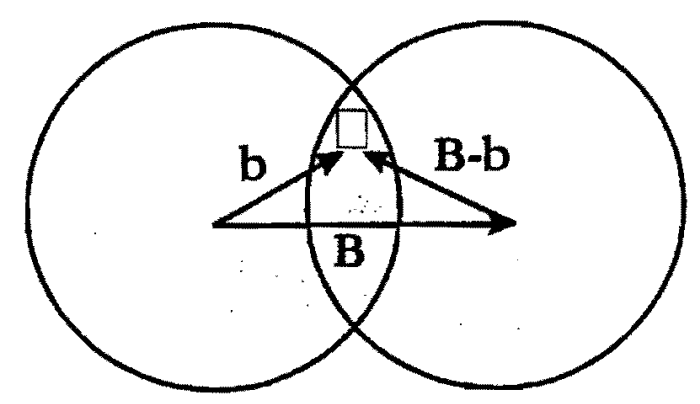

Figura 5.8: Convolução das densidades de partons no plano de impacto.

\subsubsection{Cálculo de $\left\langle|S|^{2}>\right.$}

Se $P(b)$ é a probabilidade de que dois protons passem um pelo outro com parâmetro de impacto b sem que nenhuma outra interação ocorra, além da interação dura, pode-se escrever a probabilidade de sobrevivência do gap como:

$$
<|S|^{2}>=\frac{\int F(b) P(s, b) d^{2} b}{\int F(b) d^{2} b},
$$

onde $F(b)$ é uma amplitude associada à colisão dura de interesse, obtida a partir das variáveis apresentadas na fig. (5.8) como uma convolução de densidades de partons:

$$
\begin{aligned}
\sigma_{h} & \cong \sigma_{0} \int d^{2} b d^{2} B \rho(B) \rho(b-B) \\
& \equiv \sigma_{0} \int d^{2} b F(b)
\end{aligned}
$$

donde se obtém:

$$
F(b)=\int d^{2} B \rho(B) \rho(b-B) .
$$

Dessa maneira, a probabilidade de sobrevivência do gap fica definida como sendo a probabilidade de que dois protons colidam, havendo somente uma 
interação dura (na verdade é necessário que ocorra mais uma interação, pois as partículas que restam nos feixes, após a colisão dura precisam reorganizarse para voltar a um estado neutro de cor; isso ocorre através da fragmentação, que é uma forma de interação soft. Esse ponto será retomado adiante.).

No modelo eikonal, $P(b)$ toma a forma:

$$
P(b)=\exp [-\nu \chi(b)],
$$

onde $\chi$ é a convolução de partons em si, escolhida de tal forma que $\chi(0)=1$ e $\nu$ é o parâmetro de absorção central.

No que se segue, será feita a suposição de que $F(b)$ e $\chi(b)$ podem ser aproximadas por uma Gaussiana [92] ${ }^{3}$ :

$$
\begin{gathered}
F(b)=\nu \exp \left(-\frac{b^{2}}{R_{h}^{2}}\right), \\
2 \chi(b)=2 \nu \exp \left(-\frac{b^{2}}{R^{2}}\right) .
\end{gathered}
$$

sendo $\pi R_{h}^{2}$ a área de interação para colisões parton-parton duras e $\pi R^{2}$ a área de colisões soft.

A vantagem de assumir a forma Gaussiana é o fato das integrais presentes na eq. (5.7) sejam calculdas analiticamente, resultando [92]:

$$
<|S|^{2}>=\frac{c \Gamma(c)}{\nu^{c}} \text { para } \nu>>1 .
$$

sendo $c=R^{2} / R_{h}^{2}$.

As fig. (5.9) e (5.10) apresentam $\left\langle|S|^{2}\right\rangle$ em finção de $c$ e $\nu$.

A determinação da probabilidade de sobrevivência está sujeita a ambigüidade devido a diversos fatores:

- Conforme pode-se observar na fig. (4.1), a $\sigma_{t}$ apresenta crescimento com $s$ que, supõe-se, deve manter-se para energias maiores que as testadas até o momento. Porém, não se sabe se esse aumento é ao crescimento de $\sigma_{s}$ ou de $\sigma_{h}$. Modelos diferentes assumem motivos diferentes para esse crescimento.

\footnotetext{
${ }^{3} \mathrm{O}$ modelo eikonal com essa forma Gaussiana reproduz bem os dados experimentais atualmente disponíveis para seção de choque e distribuição de partículas [70].
} 


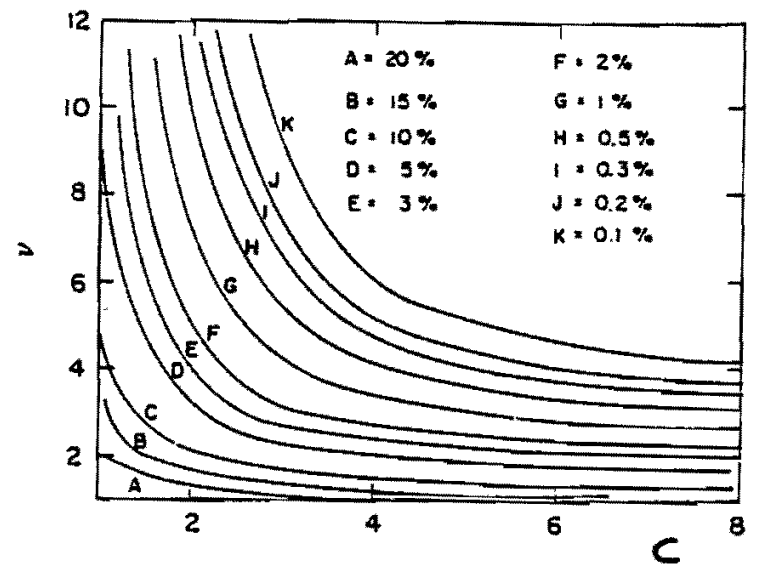

Figura 5.9: Curvas para $\left\langle|S|^{2}\right\rangle$ (apresentado na forma percentual) como função de $c$ e $\nu$.

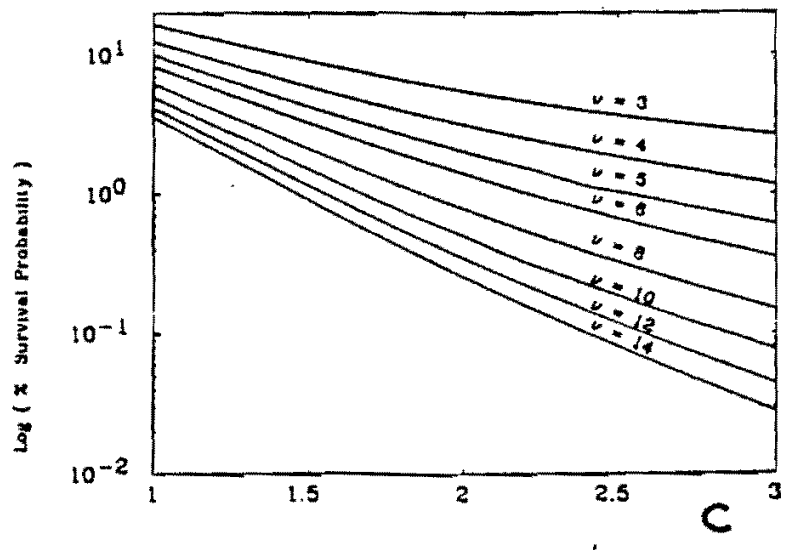

Figura 5.10: Curvas para $\log \left(\left\langle|S|^{2}\right\rangle\right)$ em função de $c$, para valores selecionados de $\nu$. 
- Observa-se da eq. (5.14) que $\left\langle|S|^{2}\right\rangle$ depende de $\nu$ e de $c$, que são determinados experimentalmente. Novamente as estimativas desses parâmetros para energias mais altas que as disponiveis atualmente dependem de modelo.

A ref. [92] faz uma avaliação de $\left\langle|S|^{2}\right\rangle$ para vários modelos, usando para isso a eq. (5.14). Um dos modelos analisados é o DPM, sendo que os parâmetros utilizados e os resultados obtidos para o LHC são:

- $\sqrt{s}=16.0 \mathrm{TeV}$.

- $\nu(s)=2.23$.

- $R_{H}^{2}=10.56 \mathrm{GeV}^{-2}$.

- $R^{2}=32.67 \mathrm{GeV}^{-2}$.

- $\sigma_{t}=109 \mathrm{mb}$.

- $\left\langle|S|^{2}>=5.3 \%\right.$.

É necessário que se faça duas observações acerca desse resultado. Em primeiro lugar, ele foi obtido partindo-se do pressuposto que a aproximação eikonal, válida até energias atualmente disponíveis, permaneça válida a energias superiores. Além disso, o cálculo não foi feito para uma colisão $p p \rightarrow W W X \rightarrow h X \rightarrow Z Z X$, mas sim para colisões $p p$ como um todo. Desta forma, a conclusão a se tirar é que, supondo-se válida a extensão da aproximação eikonal até energias próximas à do LHC, $5.3 \%$ dos eventos que ocorrerem, apresentarão somente uma colisão inelástica, mas não necessariamente um gap. Como essa proporção deve se manter sempre, então, provavelmente $5.3 \%$ das colisões do tipo $p p \rightarrow W W X \rightarrow h X \rightarrow Z Z X$ devem conter somente uma colisão inelástica, mas, novamente, não necessariamente um gap, uma vez que os remanescentes da colisão dura têm obrigatoriamente que se rearranjar, podendo dessa forma, contaminar o gap, conforme já foi discutido. É esse aspecto que é totalmente desprezado nas ref.[89, 92].

Outro trabalho [93] faz uma estimativa de $\left\langle|S|^{2}\right\rangle$ considerando a reação de interesse nesse trabalho, a partir de dois programas Monte Carlo. $O$ HERWIG [94] foi utilizado para gerar os eventos contendo o higgs, usando os dois processos que se deseja comparar, fusão de gluons e fusão de W's, 
ambos decaindo em um par $W^{+} W^{-}$. O PYTHIA foi usado para gerar alguns possiveis ruídos: $q \bar{q} \rightarrow W W, g g \rightarrow t \bar{t} \rightarrow W W$, e também $g g \rightarrow h \rightarrow W W$, esse último para que pudesse ser comparado com os resultados do HERWIG. Foi dada preferência ao HERWIG, em vez do PYTHIA, porque na época em que foi realizado o trabalho, o PYTHIA ainda não levava em consideração o ordenamento angular da radiação de gluons emitida [95], que ocorre devido a efeitos de coerência entre a radiação inicial e final. Nesta tese o HERWIG não foi utilizado por apresentar rotinas de fragmentação baseadas no Modelo de Clusters, e que apresentou resultados inferiores aos da fragmentação do Modelo LUND (além do fato do PYTHIA incluir o ordenamento angular no tratamento da emissão de radiação atualmente). O trabalho citado limitouse ao estudo do caso em que o higgs possui massa $m_{h}=500 \mathrm{GeV}$, para energia do SSC (anel de colisão $p p$ com $40 \mathrm{TeV}$ de energia no centro de massa) procurando a fração de eventos, para cada um dos processos citados acima, que não apresentava partícula na região do detetor com $|y|<2$.

Obteve, para a fusão de W's a probabilidade de sobrevivência igual a $3 \%$, para o ruído, $\left\langle|S|^{2}\right\rangle=0.01 \%$, e probabilidade de sobrevivência nula para a fusão de gluons. 


\section{Capítulo 6}

\section{Análise}

Esse trabalho tem três objetivos principais:

1. Verificar se um boson de higgs produzido pela fusão de dois bosons vetoriais produz um gap de rapidez experimentalmente detetável. Ser experimentalmente detetável implica em :

- Ser possível separar completamente o evento que possui o higgs dos eventos de fundo; esse ponto não será trabalhado nessa tese. Serão utilizadas as estratégias já disponíveis na literatura.

- Após a separação, sobreviverem ao menos alguns eventos por ano, com gap.

2. Analisar, dentro da descrição do PYTHIA a possibilidade de que um eventual gap produzido por um higgs vindo de uma fusão de gluons seja semelhante ao produzido por uma fusão de bosons vetoriais. $\mathrm{O}$ interesse desse item vem do fato de ser necessário distinguir esses dois processos de formação do higgs (vide pag. (70)).

3. Comparar a probabilidade de sobrevivência desse gap obtida a partir de duas descrições diferentes do evento subjacente à colisão dura PYTHIA (colisões múltiplas entre os constituintes dos dois protons participantes da reação) e DTUJET (troca de pomerons segundo as regras de AGK [65]. 


\subsection{A Geração dos Eventos}

\subsubsection{Algumas Informações}

\section{Massa do Quark Top}

Como o top possui uma grande massa (muito maior que as dos demais quarks), sua influência é muito grande nos diagramas com loops de quarks em que fluem energia e momento suficientes para comportá-lo. Até recentemente ele não havia sido detetado, e portanto $m_{t}$ foi sempre deixada como parâmetro nos programas geradores de eventos. Desde a sua deteção, o valor de $m_{t}$ tem sido constantemente revisto pelas colaborações D0 e CDF do FERMILAB. Entretanto, o valor utilizado nesse trabalho foi o original, $m_{t}=174$ $\mathrm{GeV}$.

\section{Funções de Distribuição}

A função de distribuição de partons dentro de um hadron, $f_{i}^{a}\left(x, Q^{2}\right)$, fornece a probabilidade de que o parton $i$ seja encontrado com fração entre $x \mathrm{e}$ $x+d x$ da energia do feixe, feixe este composto de hadrons $a$, e que tenham sido submetidos a uma colisão dura com escala de virtualidade $Q^{2}$.

Existem várias funções de distribuição publicadas na literatura, produzidas por ajustes diferentes dos dados experimentais, em diferentes ordens de teoria de perturbação e diferentes esquemas de renormalização. Nesse trabalho foi utilizado os ajustes propostos pela Colaboração CTEQ na ref. [96]. O PYTHIA possue implementado o ajuste L2, enquanto o DTUJET possue o ajuste L1.

\section{Calorimetro}

Num experimento, a energia de partículas hadrônicas, fotons e eletrons $\left(e^{+} \mathrm{e}\right.$ $e^{-}$) são colhidas respectivamente,por calorímetros hadrônicos e eletromagnéticos, que no caso de colliders, podem ser convenientemente mapeados pelas coordenadas esféricas dadas pelo ângulo polar $\theta$ e o ângulo azimutal, $\phi$. Porém, usualmente, no lugar de $\theta$, usa-se a rapidez, definida por $y=\frac{1}{2} \log \frac{E+P_{z}}{E-P_{z}}$, que no limite de altas energias tem os mesmos valores que a pseudo-rapidez $\eta=\log \tan \frac{\theta}{2}$. A vantagem da troca de $\theta$ por $y$, é que assim pode-se trabalhar somente com grandezas invariantes por translações ao longo do eixo $z$, em 
cuja direção os feixes se deslocam. No presente trabalho, geralmente será usada a rapidez, sendo feita uma ressalva nos pontos em que ela for substituída pela pseudo-rapidez.

Neste trabalho, é utilizada uma idéia simplificada do calorímetro. Não é feita diferença entre calorímetro hadrônico e eletromagnético, e com $100 \%$ de resolução. Sua área interna do calorímetro é dividida em células, cada uma com dimensão $\Delta y \times \Delta \phi$, sendo $\Delta y$ dado pela cobertura em $y$ que o calorímetro é capaz de dar, dividido pelo número de segmentações que ele possui; $\Delta \phi$ é definido como $2 \pi$ dividido pelo número de segmentações.

O trabalho foi desenvolvido considerando-se em termos gerais um acelerador de protons como o LHC (Large Hadron Collider), com $14 \mathrm{TeV}$ de energia no centro de massa, e com luminosidade $\mathcal{L}=10^{34} \mathrm{~cm}^{-2} \mathrm{~s}^{-1} \approx 100 \mathrm{fb}^{-1} \mathrm{ano}^{-1}$.

Porém não foram levados em conta detalhes específicos dos detetores, como forma, cobertura, efeito do material de que é constituído, resolução, segmentação, etc. Foi simulado com o Pythia um calorímetro simplificado, com as seguintes características:

cobertura de rapidez: $-5<y<5$

segmentação: A área total do calorímetro é subdividida em células de dimensão $y \times \phi=\frac{10}{50} \times \frac{2 \pi}{30}=0.2 \times \frac{1}{12}$.

\section{Hadronização}

A subrotina usada para a definição dos jatos é a LUCELL, do Pythia, descrita a seguir de forma extremamente simplificada.

As células com energia transversal depositada superior a $E_{T_{m i n}}$ são ordenadas em ordem decrescente de energia transversal. Soma-se a energia transversal $\left(E_{T}=E \operatorname{sen} \theta\right)$ depositada nas células que distam até um dado $\Delta R$ dela, sendo $\Delta R$ calculado como $\sqrt{(\Delta y)^{2}+(\Delta \phi)^{2}}$, se essa soma for superior a $E_{T_{\text {total }}}$, aquele grupo de células é considerado um jato (todas as células que fazem parte desse jato e que porventura estivessem na lista ordenada de energia transversal, são retiradas dela). Se for inferior, tenta-se novamente o mesmo algoritmo com a próxima célula (proveniente da ordenação decrescente em $E_{T}$ ), até que não reste nenhuma célula com energia transversal superior a $E_{T_{m i n}}$.

Os parâmetros utilizados com o Lucell nesse trabalho, foram: 
$E_{T_{\min }}=1.0 \mathrm{GeV}$.

$\Delta R=\sqrt{(\Delta \eta)^{2}+(\Delta \phi)^{2}}=0.7$.

$E_{T_{\text {total }}}=7.0 \mathrm{GeV}$.

\subsubsection{Seleção dos Eventos}

Um dos principais objetivos dos trabalhos de simulação executados antes de uma experiência é a seleção dos cortes que devem ser impostos aos eventos obtidos, de forma a separar o sinal da partícula ou reação desejada de todas as outras partículas e reações que ocorrerão, e que recebem o nome genérico de ruído de fundo. Essa separação deve ser feita com a maior eficiência possível (ou seja, perdendo a menor quantidade possível de sinal).

Vários fatores interferem na estratégia a ser adotada na análise de uma reação, dentre eles a luminosidade do acelerador, a energia dos feixes incidentes, a segmentação dos detetores, sua resolução e eficência, o mecanismo de produção da partícula de interesse, seus canais de decaimento e no caso do higgs, a sua massa.

Além disso, sem dúvida, antes de se determinar a forma de análise a ser empregada, é preciso definir o que se considera sinal e ruído.

\section{Definição do Sinal}

A reação procurada nesse trabalho é o processo $W^{+} W^{-} \rightarrow h_{0}$, ou seja, a produção de higgs por fusão de W's. A reação $Z^{0} Z^{0} \rightarrow h_{0}$ também interessa, pois pode gerar os mesmos estados finais que a primeira, e possui todas as mesmas características que ela. Ela não foi incluida entre os eventos gerados, pois não seria introduzida nenhuma diferença na análise. A escolha do canal de decaimento do higgs é importante, porque há alguns extremamente difíceis de serem observados, embora muitas vezes tenham uma seção de choque considerável.

Foi imposto ao higgs o decaimento num par de bosons Z, que por sua vez decaíram em pares de muons; os motivos dessa escolha são vários:

- Os bosons Z são singletos de cor, de forma que não emitirão gluons, que produziriam jatos fazendo com que o gap desaparecesse. 
- Seu decaimento em um par de muons não produzirá hadrons. Esse aspecto é importante, pois decaimentos envolvendo jatos contaminariam um gap que porventura existisse, ficando difícil distinguir um evento que não produziu o gap de outro que teve seu gap preeenchido pelo decaimento do higgs. Poderiam ser utilizados eletrons em vez de muons, do ponto de vista cinemático, nada se modificaria. Do ponto de vista experimental os muons possuem a vantagem de apresentar um ruído de fundo menor, o que é crucial num acelerador que trabalhará com a luminosidade do LHC.

- Esse produto final do higgs contendo quatro leptons carregados é conhecido como gold plated pelo fato de ser muito limpo. Os leptons produzidos são isolados, e a massa do higgs pode ser medida diretamente como a massa invariante produzida por esses leptons. Mas infelizmente, a razão de ramificaçăo desse decaimento é muito pequena (da ordem de $0.07 \%$ para $h_{0} \rightarrow \mu^{+} \mu^{-} \mu^{+} \mu^{-}$. Como se está tratando de um processo com seção de choque da ordem de picobarns, a taxa de eventos produzidos anualmente é pequena.

- Além disso a eficiência das câmaras de muons no reconhecimento dessas partículas nos experimentos atuais é muito alta, da ordem de 98 a $99 \%$.

- Existe a possibilidade de que um hadron (em geral pions e kaons) devido à sua longa vida média, chegue às câmaras de muons, e seja confundido com um muon pelo calorímetro ( punch through). A probabilidade de que isso ocorra cresce com a energia do hadron, e para kaons e pions com $120 \mathrm{GeV}$ de energia, a probabilidade de que ocorra punch through é de $\approx 3 \%$ [97].

\section{Características do Processo $W^{+} W^{-} \rightarrow h_{0} \rightarrow Z_{0} Z_{0}$}

Há várias características do processo de fusão de bosons W que auxiliam na tarefa de separar o sinal do ruído:

- O produto final, constituído de quatro muons isolados (os muons do ruído de fundo são provenientes de decaimentos semi-leptônicos de quarks pesados, e portanto estão sempre acompanhados de jatos) e 


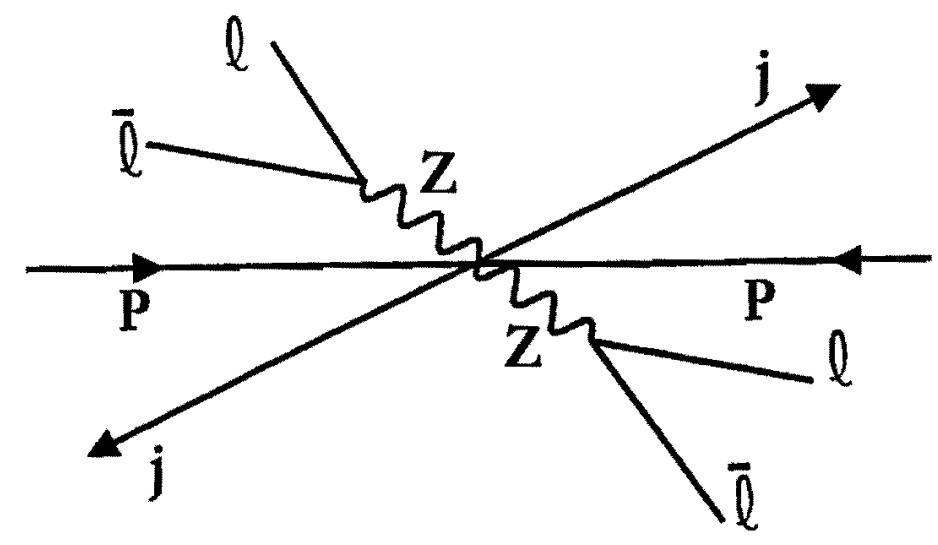

Figura 6.1: $O$ processo $W W \rightarrow h \rightarrow Z Z$ no referencial de repouso do higgs.

altamente energéticos, representa uma assinatura bastante clara dessa reação, permitindo a reconstrução da massa dos Z's que os emitiram, e subseqënte reconstrução da massa do higgs.

- Conforme já foi mencionado (vide pag. (41)), os bosons Z provenientes do decaimento de um higgs com massa superior a $2 m_{W}$ têm polarização predominantemente longitudinal, enquanto a polarização dos Z's provenientes de $q \bar{q} \rightarrow Z Z$ é, na maior parte das vezes, transversal. Atualmente há certo ceticismo quanto a possibilidade de se usar a polarização como assinatura, devido à falta de sinais experimentais que ela gera.

- Os dois quarks que emitem os bosons vetoriais produzem dois jatos próximos aos feixes (grande rapidez), e com momento transversal da ordem de $M_{W} / 2$ (vide pag. (46)), conforme se observa nas fig. (5.1) e 6.1. Esses dois jatos podem ser utilizados num tag. Nem a fusão de gluons nem os processos de QCD produzem esses jatos.

- A própria possibilidade de existência do gap (discutida no cap. (5)) é uma importante característica desse tipo de processo. 


\section{Definição do Ruído}

No caso do higgs produzido por fusão de bosons vetoriais $\mathrm{W}$ num collider hadrônico, há três tipos de ruídos a serem considerados:

- Os decaimentos do higgs precisam ser separados do evento subjacente, ou seja, dos produtos das reações dos gluons,quarks e diquarks que formavam o proton, e que restaram após a colisão dura. É possivel, por exemplo, que um dos produtos intermediários do feixe remanescente seja um quark $b$, cujos decaimentos semi-leptônicos produzem leptons que podem ser confundidos com os do sinal, levando a uma massa reconstruída errada para o higgs. Esse tipo de problema é contornado com um corte de isolamento, que exige que não haja hadrons muito energéticos num cone próximo aos leptons.

- O evento contendo um higgs precisa ser distinguido de outros eventos que produzam o mesmo estado final composto de quatro leptons.

As principais reaçồes com esse perfil são:

$p p \rightarrow Z Z$ conhecido como contínuo $Z Z$, é irredutível por ter o mesmo estado final que o sinal. Essa reção inclui vários processos: $W W \rightarrow$ $Z Z, Z Z \rightarrow Z Z, q \bar{q} \rightarrow Z Z, g g \rightarrow Z Z$.

$p p \rightarrow Z Q \bar{Q}$ sendo $Q=b$ ou $t$, que podem produzir leptons.

$p p \rightarrow t \bar{t} \rightarrow W^{+} b W^{-} \bar{b}$ onde os bosons $\mathrm{W}$ decaem em leptons e os jatos provenientes do quark $b$ simulam leptons isolados.

- No caso específico desse trabalho, deseja-se analisar a possibilidade de separar o higgs produzido por uma fusão de W's do produzido pela fusão de gluons. Portanto, considerando-se como sinal a fusão de W's, o higgs produzido por uma fusão de gluons é um ruído, e como tal deve ser separado do sinal.

\subsubsection{Processos Analisados}

Para atingir os objetivos descritos, foram gerados eventos de produção de bosons de higgs com massas entre 300 e $900 \mathrm{GeV}$, variando de $100 \mathrm{em} 100$ $\mathrm{GeV}$, para uma energia no centro de massa igual a $14 \mathrm{TeV}$.

Os eventos foram gerados da seguinte forma: 


\section{Com o código Pythia}

fusão de W's: Para a geração dessa reação foi usado o processo 124 do PYTHIA, que é baseado na ref. [85]. O higgs é descrito como uma ressonância, sendo usada uma aproximação de Breit-Wigner, de forma que os reultados obtidos para o higgs com massa até $400 \mathrm{GeV}$ descrevem fielmente a física envolvida. A partir dessa massa, até $700 \mathrm{GeV}$ a descrição é uma boa aproximação. Para massas maiores que $700 \mathrm{GeV}$, os resultados obtidos não são bons, e foram mantidos apenas para que se possa ter uma ligeira visão do que ocorreria nesses casos.

fusão de gluons: Para a geração desse processo foi usada a reação 102 do PYTHIA [98]. Na seção (3.3.4) é apresentado um cálculo da sua seção de choque. É usada aqui também Breit-Wigner.

2. Fusão de W's com o código DTUJET, embora esse programa não possua a reação de fusão de bosons $\mathrm{W}$ formando um higgs. Os bosons W e Z, e também o higgs não foram incluídos ainda nesse programa. Segundo o modelo adotado pelo DTUJET, ao emitir um boson W, o proton divide-se irremediavelmente em dois objetos independentes: um quark (que emitiu o boson) e um diquark, e no caso de uma colisão proton-proton, esses quatro elementos remanescentes da colisão interagirão da mesma forma que um processo de minimum bias, trocando, em primeira aproximação, um pomeron que formará duas cadeias de hadrons, ou dois strings. Porisso o procedimento adotado foi o seguinte:

- Para cada uma das massas de higgs consideradas foi rodado um programa com o Pythia, produzindo 10 mil eventos, sendo guardada, para cada evento gerado, a energia $\sqrt{\hat{s}}$ consumida pelo subprocesso (a fusão de W's). Para cada uma das massas foi calculado o valor médio dessa energia, $\langle\sqrt{\hat{s}}>$.

- Novamente para cada uma das massas, foi gerado, com o DTUJET, um grupo de 10 mil eventos, sendo que a energia no centro de massa foi calculada como: $\sqrt{s}=14 T e V-<\sqrt{\hat{s}}\rangle$. Os eventos foram gerados de modo a obedecer à condição de não haver difração, pois não seria possível haver uma colisão difrativa, e além disso uma fusão de bosons $\mathrm{W}$. Não está sendo considerada aqui 
a possibilidade de difração dura (sec. 4.1.1). O programa inclui somente difracão soft.

Não foi gerada fusão de gluons com esse código.

É necessário lembrar que dessa forma não foram produzidos os jatos na direção do feixe, e com momento transversal da ordem da massa do boson W, cuja fragmentação poderia, eventualmente, contaminar um gap que estivesse presente no processo. Esse fato afeta o resultado final.

\section{Cortes no Espaço de Fase}

No caso da fusão de bosons vetoriais foi introduzida uma exigência no valor mínimo do momento da colisão dura com a intenção de acelerar a geração de eventos. Como não seria interessante alterar a amostra de eventos obtida, foi realizado um teste com a geração de mil eventos, cujo resultado é mostrado na fig. (6.2), que consta de um histograma do momento transversal trocado nas reações para três massas diferentes do higgs $(300,500$ e $900 \mathrm{GeV})$. A partir desses resultados, os cortes foram escolhidos como sendo:

- para $m_{h}=300 \mathrm{GeV}$ ou $m_{h}=400 \mathrm{GeV}$, momento transversal mínimo $p_{t_{\min }}=100 \mathrm{GeV}$.

- para $m_{h}=500 \mathrm{GeV}, m_{h}=600 \mathrm{GeV}$ ou $m_{h}=700 \mathrm{GeV}$, momento transversal mínimo $p_{t_{\min }}=150 \mathrm{GeV}$.

- para $m_{h}=800 \mathrm{GeV}$ ou $m_{h}=900 \mathrm{GeV}$, momento transversal mínimo $p_{t_{\min }}=200 \mathrm{GeV}$.

Já para a produção de higgs por fusão de gluons, como se observa na fig. (6.3), não pode ser feito um corte assim tão alto. Nesse caso foram gerados eventos com $p_{t_{\min }}=5 \mathrm{GeV}$.

Esses cortes não foram usados com o DTUJET, pois o subprocesso de fusão de W's não foi efetivamente gerado.

\subsubsection{Cortes Adotados para Seleção dos Eventos}

As diferentes características do sinal e do ruído são usadas para separá-los um do outro, através da implementação de cortes. Por exemplo, como os muons 


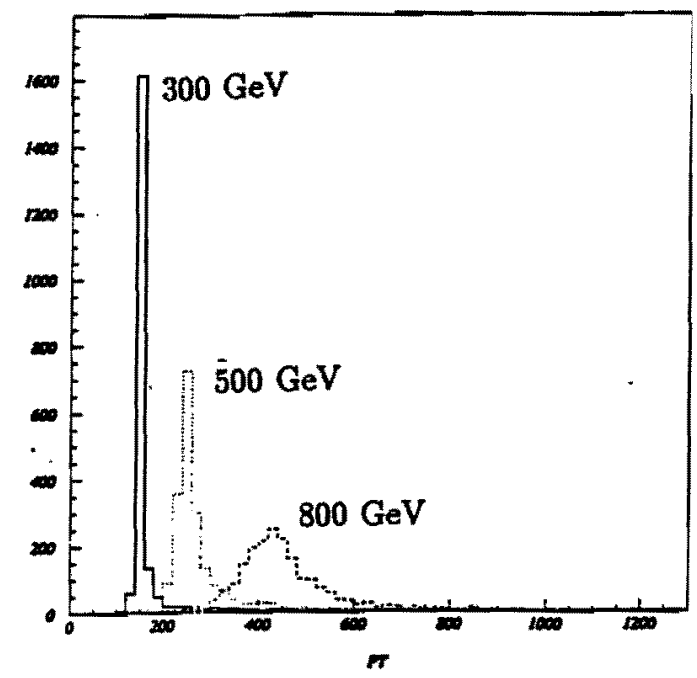

Figura 6.2: Distribuição do momento transversal trocado na fusão de bosons W produzindo higgs com massas de $300 \mathrm{GeV}, 500 \mathrm{GeV}$ e $800 \mathrm{GeV}$.

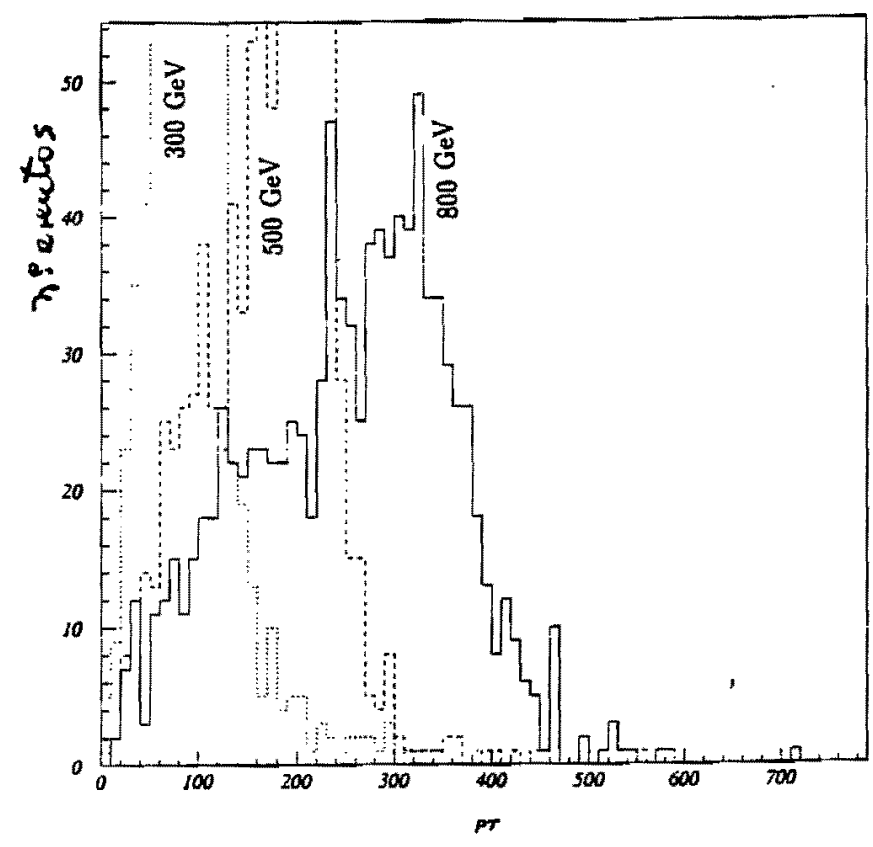

Figura 6.3: Distribuição do momento transversal trocado na fusão de gluons produzindo higgs com massas de $300 \mathrm{GeV}, 500 \mathrm{GeV}$ e $800 \mathrm{GeV}$ 
vindos do decaimento do higgs são produzidos isolados, são cortados (ou eliminados) os eventos que não apresentem essa característica. No presente trabalho os cortes adotados foram divididos em dois conjuntos.

De um lado, foram considerados os cortes relativos aos leptons, levando em consideração suas características de isolamento, energia, a massa invariante que eles carregam, etc. Esses cortes foram agrupados em um conjunto denominado Conjunto de Cortes Comuns, e foi aplicado a todos os eventos gerados. A intenção básica desse grupo de cortes é separar um evento contendo um higgs decaindo pelo canal $h_{0} \rightarrow Z_{0} Z_{0} \rightarrow \mu^{+} \mu^{-} \mu^{+} \mu^{-}$de todos os outros que, ou nem sequer produzem quatro muons, sendo completamente diferentes do sinal, ou provêm de algumas das outras reações citadas anteriormente (sec. (6.1.2)) que contêm muons provenientes de decaimentos de quarks pesados, ou outras fontes que não sejam o Z.

O objetivo de todo corte é aumentar a razão sinal/ruído, pois seria impossível eliminar o ruído sem causar dano ao sinal. Um exemplo disso é o corte de isolamento dos quatro muons que, dos cortes apresentados até agora, é o que mais afeta o sinal, e no entanto, é essencial para eliminar os muons provenientes de quarks.

Como não há esperança de se eliminar completamente o $\mathrm{ZZ}$ contínuo, coloca-se num mesmo gráfico a massa reconstruída a partir dos quadrimomentos dos leptons finais para todos os eventos que tenham passado pelos cortes apresentados na seção anterior. A maior parte desses eventos é constituída pelo contínuo $\mathrm{ZZ}$ e vai produzir uma linha aproximadamente suave em grande parte da extensão do espectro; espera-se que o higgs, sendo uma ressonância, produza um pico nessa figura em torno da região de sua massa (ver para ilustração a fig. (6.4)). Porém, o número de eventos produzindo higgs é muito pequeno, e esse pico com certeza será muito sutil. No caso de um higgs muito pesado $\left(m_{h} \sim 600 \mathrm{GeV}\right)$, que é também muito largo (pag. (41)), não será possível determinar a massa dessa forma. Neste caso será necessário de alguma maneira cercar mais o sinal, mesmo que perdendo uma fração maior do mesmo, mas tendo uma maior confiabilidade nos que permanecerem.

Surge então o outro conjunto de cortes, onde foram colocados os que lidam especificamente com a diferença entre processos que produzam um par de Z's decaindo em quatro muons, sejam contínuo $\mathrm{ZZ}$, fusão de gluons levando a higgs, ou qualquer outro, pois baseiam-se no tag dos quarks que emitiram os bosons W. Para este segundo conjunto de cortes existe muita divergência na literatura, motivo pelo qual foram consideradas três possibilidades diferentes. 

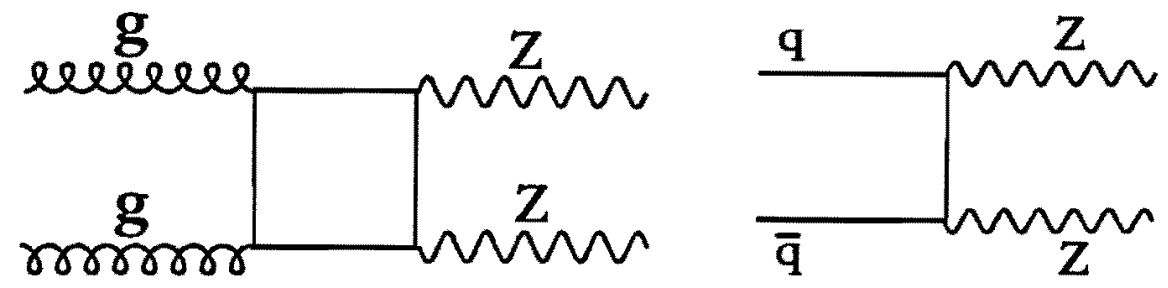

Figura 6.4: Diagrama para o contínuo ZZ.

Esse conjunto foi denominado Conjunto de Cortes Específicos.

Todos os cortes pincelados aqui serão descritos em detalhes nos próximos itens.

\section{Conjunto de Cortes Comuns}

Todos os eventos produzidos pelo Pythia e analisados nesse trabalho, passaram pelo seguinte conjunto de cortes, que foi chamado de Conjunto dos Cortes Comuns $[100,101]$ :

1. Os leptons devem ter: $\left|y^{l}\right|<2.5$ e $p_{T}^{l}>10 \mathrm{GeV}$. A maioria das publicações exige que os quatro leptons cumpram essa condição, que nesse trabalho foi relaxada. Foi exigido que os quatro leptons possuíssem $p_{T}^{l}>10 \mathrm{GeV}$, mas apenas três deles precisavam ter $\left|y^{l}\right|<2.5$.

2. Cada um dos leptons deve ser isolado. Uma medida desse isolamento é exigir que numa região de raio $R=\sqrt{\phi^{2}+y^{2}}=0.3$ em torno do eletron, a energia transversal depositada não ultrapasse o limite de $E_{T}^{\max }=5 \mathrm{GeV}$.

3. Considerando-se os quatro leptons, deve ser possivel formar dois pares cujas massas invariantes sejam próximas da massa do Z, ou seja, $\mid M_{l l}-$ 


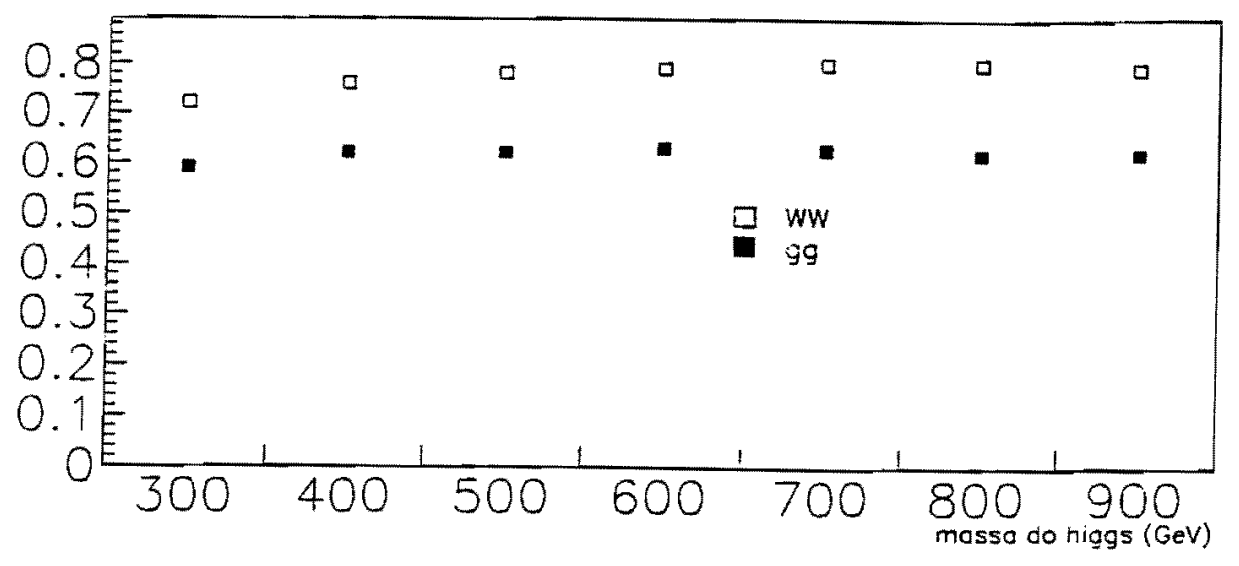

Figura 6.5: Fração dos eventos que sobrevivem ao Conjunto de Cortes Comuns.

$M_{Z} \mid<10 \mathrm{GeV}$. O objetivo desse corte é eliminar os pares de muons que não sejam provenientes do decaimento de Z's.

4. Identificação do muon (que é feita corretamente em aproximadamente $98 \%$ dos casos).

5. Ao menos um $Z$ obedecendo a condição: $p_{Z}>\sqrt{M_{Z Z}^{2}-4 M_{Z}^{2}}$, onde $M_{Z Z}$ é a massa invariante do par de Z's, ou seja, a massa do higgs produzido. $\mathrm{O}$ objetivo desse corte é eliminar ruído devido à QCD.

A fig. (6.5) apresenta a fração dos eventos que sobrevivem ao conjunto de cortes exposto acima, em função da massa do higgs.

\section{Conjunto de Cortes Específicos}

Foram criados quatro grupos de eventos, Grupo I, Grupo II, Grupo III e Grupo IV. Os Grupos I, II e III são constituídos, todos eles, de 10 mil eventos (gerados com o PYTHIA para cada uma das massas de higgs consideradas, $300,400,500,600,700,800$ e $900 \mathrm{GeV}$, produzidos por fusão de gluons e por fusão de W's. O que diferencia os três grupos são os cortes a que os eventos 
foram submetidos. O Grupo IV é constituído de 10 mil eventos gerados com o DTUJET. Os quatro grupos são descritos a seguir.

Grupo I O primeiro deles procura levar em conta a sugestão de Björken [89], analisada no cap. (5), e faz um tag em dois jatos do evento, para verificar se há um gap de rapidez entre eles. A princípio esse não é um corte eficiente porque, embora reduza bastante o ruído, reduz também o sinal, levando a uma razão sinal/ruído menor que a obtida com outros recursos. Porém, existia a posibilidade de que tal corte evidenciasse de tal forma a existência do gap de rapidez, que seu uso se tornasse interessante; ou então, que esse corte, aliado a algum outro baseado no gap, levasse a uma melhor relação sinal-ruído.

Os cortes empregados nesse grupo são:

1. O conjunto de cortes comuns a todos os eventos, apresentados na pag. (98).

2. Exigência de que existam no mínimo dois jatos com momento transversal de $40 \mathrm{GeV}$ e $|y|>2$, sendo $\eta$ a rapidez do jato. Essas escolhas são justificadas pelo explanado anteriormente: o jato originado pelo quark que emite o $\mathrm{W}$ adquire um momento transversal da ordem de $m_{W} / 2$, e segue a direção dos feixes incidentes, ou seja, tem grande rapidez.

Define-se o tamanho do intervalo onde é procurado o gap como a distância em rapidez entre os dois feixes que emitiram os bosons $W$, dada por: $\Delta(y)=y_{1}-y_{2}-1.4$, conforme pode ser observado na fig. (5.3) onde $y_{1}$ e $y_{2}$ são dados pela rapidez dos dois jatos que satisfazem as condições impostas acima. Se mais que dois jatos satisfizerem essas condições são considerados os dois com maior momento transversal. É subtraído o valor 1.4 porque considera-se que cada um dos jatos possua um raio de 0.7 unidades no plano $y \times \phi$.

Grupo II Nesse grupo são usados os cortes propostos nas ref. [101]; é feito um tag em somente um dos jatos, levando-se em consideração a observação de que fazer um tag em dois jatos diminui muito a estatística, embora melhore a razão sinal/ruído ( [101]).

Os cortes incluídos nesse grupo são:

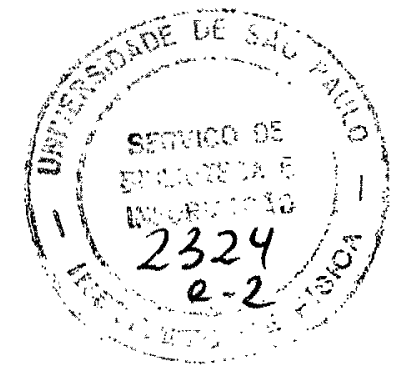


- O conjunto de cortes comuns a todos os grupos, apresentados na pag. (98).

- O evento deve possuir ao menos um jato com energia $E>1 \mathrm{TeV}$ e $2.0<|y|<5.0$.

Para os processos submetidos a esse corte, não é possível verificar se há ou não gap da mesma maneira que foi feita para os eventos do Grupo $\mathrm{I}$, pois não é feito um $\operatorname{tag} \mathrm{em}$ dois jatos.

Por isso, os gaps são procurados em dois intervalos fixos, simétricos em relação ao zero. Um deles vai de $y=-2$ a $y=2$, e o outro de $y=-3$ a $y=3$, e portanto os intervalos possuem larguras constantes respectivamente iguais a $\Delta y=4$ e $\Delta y=6$. É necessário observar que o intervalo de rapidez de largura $\Delta y=6$ já traz de início um problema, porque o tag efetuado admite jatos com rapidez entre $2.0 \mathrm{e}$ 5.0 , que portanto muito provavelmente sujarão o intervalo. A intenção foi somente a de analisar a possibilidade de existência de um $g a p$ com mais do que quatro unidades de rapidez.

Grupo III Nesse grupo os eventos são gerados com o PYTHIA, e não são feitos cortes além dos apresentados na sec. (6.1.4). Esse é o tipo de procedimento que foi adotado pelo GEM [100] em sua análises para o SSC. Foi usado aqui, embora se esteja tratando de um acelerador com energia menor no centro de massa (LHC), porque os cortes adotados no Conjunto de Cortes Comuns refere-se somente a produtos da colisão dura, gerados a partir do higgs, e que portanto não dependem da energia total disponivel, mas somente de $m_{h}$.

Também nesse caso, os gaps são procurados em dois intervalos fixos, simétricos em relação ao zero. Um deles vai de $y=-2$ a $y=2$, e o outro de $y=-3$ a $y=3$, e portanto os intervalos possuem valores constantes respectivamente iguais a $\Delta y=4$ e $\Delta y=6$.

Grupo IV Os eventos desse grupo foram gerados com o DTUJET, conforme detalhado no início desse capítulo (pag.( 94)). Não foram feitos cortes, porque na verdade, como discutido anteriormente, o higgs não é produzido nesse gerador, e não há como fazer a sua identificação. 


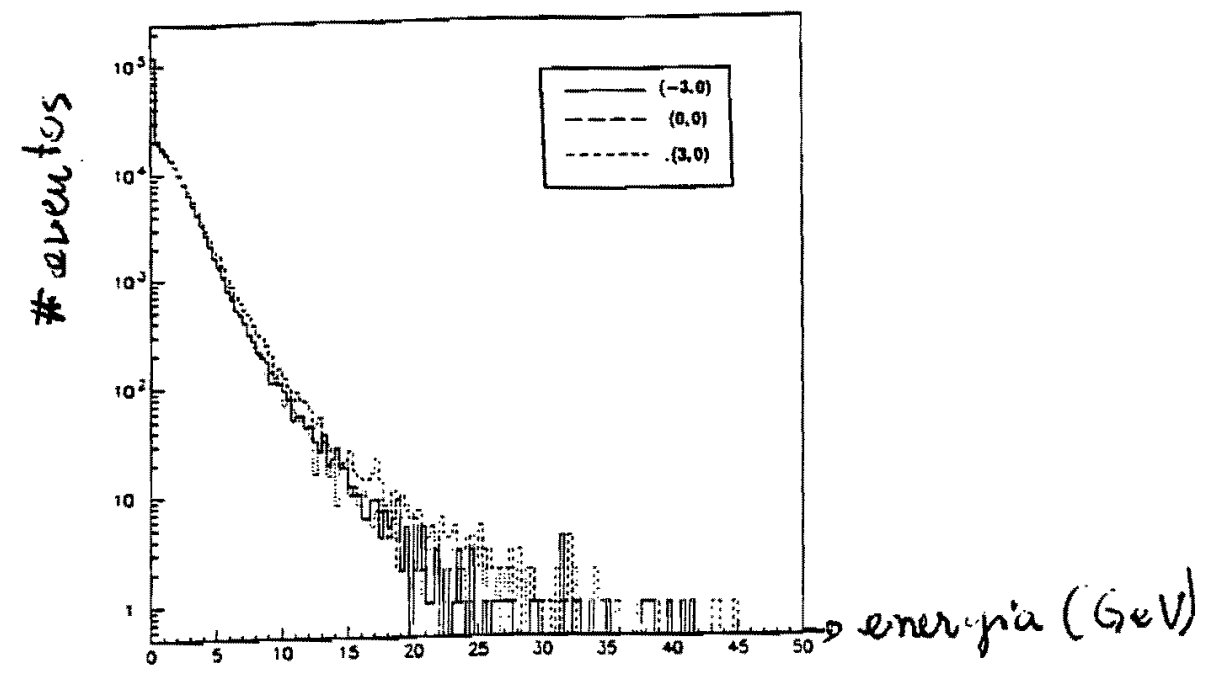

Figura 6.6: Distribuição de energia depositada em cones centrados em três pontos no espaço $y \times \Phi:(-3,0),(0,0),(3,0)$.

\subsection{Pile Up}

Conforme apresentado na sec. ( 6.2), devido à alta luminosidade dos anéis de colisão, a cada bunch crossing ocorrerão várias colisões proton-proton. Nesta seção será apresentado o método utilizado na geração e análise de eventos de pile up [1.02].

Os eventos foram gerados com o PYTHIA sendo usados os mesmos parâmetros apresentados na $\sec (6.1 .1)$. Foram incluídos todos os processos possíveis em uma colisão proton-proton.

Foram gerados 250 mil eventos, e somada a energia depositada em cones com raio $R=\sqrt{(\Delta y)^{2}+(\Delta \Phi)^{2}}=0.7$ em torno dos seguintes pontos no espaço $y \times \Phi:(y, \Phi)=(-3,0),(-2,0),(-1,0),(0,0),(1,0),(2,0),(3,0)$, abrangendo assim a região de interesse nesse trabalho (no que concerne a $y$; em relação a $\Phi$, a distribuição deve ser uniforme). Desta forma foi formado um banco de eventos.

A fig. ( 6.6) mostra a distribuição de energia depositada nos cones centrados em três pontos diferentes: $(-3,0),(0,0) e(3,0)$. As três distribuições são semelhantes, mostrando que na região central de rapidez a distribuição de partículas é uniforme. 
Supondo-se que cada uma das colisões proton-proton seja independente das demais ocorridas no mesmo bunch crossing, pode-se usar uma distribuição de Poisson com média $N$ para obter o número de colisões num determinado bunch. Isso foi feito para dois valores de $N: 8$ e 12. Para cada uma dessas médias, após ser escolhido o número de colisões $n_{c o l}$ de um determinado pile up, foram escolhidos aleatoriamente $n_{c o l}$ eventos do banco de dados formado anteriormente. Desta forma, foram gerados 10 mil eventos com pile up. Aproximadamente $20 \%$ desses eventos possui jatos (ou cones) com mais de $20 \mathrm{GeV}$ de energia depositada. Este resultado está de acordo com outro publicado recentemente [103].

Esse fato implica em grande contaminação dos eventuais gaps formados por algum desses eventos. 


\section{Capítulo 7}

\section{Resultados e Discussões}

A sec.( 5.2) mostra que no estudo da deteção do gap foi dada ênfase à determinação da Probabilidade de Sobrevivência do Gap de Rapidez, $S$, definida como a fração de eventos que contém efetivamente o gap dentre todos os eventos potencialmente geradores de um gap.

\subsection{Probabilidade de Sobrevivência do Gap}

A fig. (7.1) compara a Probabilidade de Sobrevivência do Gap nos casos de fusão de gluons e de fusão de W's, em função da massa do higgs, quando foram aplicados aos eventos os cortes do Grupo I. Não foram consideradas colisões múltiplas, ou seja, apenas um parton de um dos protons vai interagir com somente um parton do outro proton.

Cada um dos pontos dessa figura é obtido da seguinte forma:

1. Para cada massa do higgs considerada, e para cada um dos processos, foi gerado o número de eventos necessário para que, após serem impostos os cortes do Grupo I, restassem 10 mil eventos.

2. Para cada um desses 10 mil eventos, foi calculado o tamanho e a localização do gap de rapidez da maneira proposta por Björken [89] (pag.(100)).

3. Depois disso são contados quantos eventos mantêm a existência do gap, ou seja, qual o número de eventos $N_{g a p}$ em que fica vazia a região destinada ao gap em três situações: 
(A) Foram levadas em consideração todas as partículas finais carregadas, com exeção dos muons.

(B) Foram consideradas as partículas finais carregadas, com exceção dos muons, que tivessem um momento transversal $p_{T}>1.0 \mathrm{GeV}$.

(C) Foram consideradas as partículas finais carregadas, com exceção dos muons, que tivessem um momento transversal $p_{T}>2.0 \mathrm{GeV}$.

4. Esse número $N_{g a p}$ foi dividido pelo número total de eventos gerados (antes de se fazer uso de qualquer um dos cortes), produzindo então $S_{i n i}$, nas três situações diferentes (A), (B) e (C) descritas acima.

5. Separadamente, para cada uma das massas do higgs, e para cada um dos processos de produção (fusão de W's e de gluons) foi gerado um grupo de 10 mil eventos aos quais foram aplicados os cortes comuns a todos os eventos (pag. (98)); foi calculada, para cada um dos dois processos, fração de eventos que sobrevive a esse grupo de cortes. Esa fração foi multiplicada pelo valor de $S_{i n i}$, obtido no item anterior, obtendo-se assim, $S$, a probabilidade de sobrevivência do gap para cada um dos casos considerados.

Observa-se na fig.( 7.1):

- De acordo com as previsões teóricas, a probabilidade de sobrevivência do gap é maior na fusão de W's do que na fusão de gluons;

- Para a fusão de W's, $S$ é uma ordem de grandeza menor que o esperado nos cálculos teóricos, que gira entre 5 e $10 \%$. Esse comportamento já era esperado, pois já foi comentado que os estudos teóricos levam em conta a probabilidade de que haja interações múltiplas entre os partons constituintes dos protons, mas não consideram a possibilidade da fragmentação dos elementos remanescentes dos protons, mesmo no caso de uma interação simples, após a colisão, atingirem a região central do detetor.

- A probabilidade de sobrevivência do gap é quase nula no caso da fusão de gluons, mas ela é nula somente na parte (A) da figura; nas outras duas partes ela é muito pequena, mas diferente de zero. 


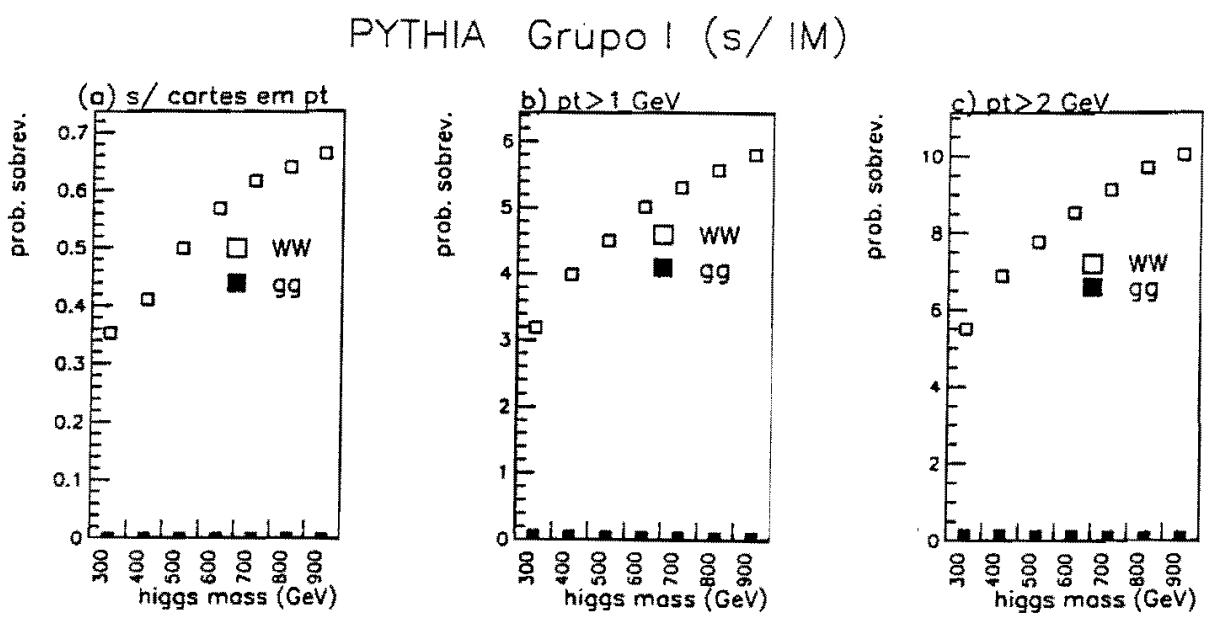

Figura 7.1: Probabilidade de Sobrevivência do Gap na formação de um higgs por fusão de W's e de gluons. O texto esclarece as legendas. As situações (A), (B) e (C) estão descritas na pag. (104). IM=Interações Múltiplas.

- Se forem consideradas somente as partículas finais com momento transversal $p_{T}>1.0 \mathrm{GeV}$, obtém-se a parte (B) da mesma figura, em que a probabilidade de sobrevivência do gap para a fusão de W's varia entre $3 \%$ para $m_{h}=300 \mathrm{GeV}$ e aproximadamente $6 \%$ para $m_{h}=900 \mathrm{GeV}$, e mantém-se muito pequena (mas não nula) para a fusão de gluons.

- A parte (C) da mesma figura mostra a probabilidade de sobrevivência do gap no caso em que somente partículas finais com momento transversal $p_{T}>2.0 \mathrm{GeV}$ são consideradas, e chega-se a obter $S \sim 10.0 \%$. Novamente obtém-se para a fusão de gluons valores muito próximos de zero (mas que não são nulos).

- Nos três casos apresentados, observa-se que a probabilidade de sobrevivência do gap aumenta com a massa do higgs, para a fusão de W's, chegando a resultados bastante animadores para higgs pesados.

Porém, se forem levadas em conta as colisões múltiplas, obtemos os resultados apresentados na fig.(7.2). Os pontos dessa figura foram obtidos exatamente da mesma forma que os da figura anterior, com a única diferença 
de que foi considerada a possibilidade de que ocorram interações múltiplas, de acordo com o modelo descrito na sec. (5.1.2) na geração dos eventos. A observação dessa figura leva às seguintes conclusões:

- A porcentagem de eventos sobreviventes é muito menor que no caso anterior, onde não eram consideradas as colisões múltiplas.

- Observa-se na parte (A) da fig. que a probabilidade de sobrevivência do gap é bastante pequena, chegando no máximo a $0.004 \%$ dos eventos de produção de higgs por fusão de W's, quando são consideradas todas as partículas finais carregadas (com exceção dos muons).

- Se forem consideradas somente as partículas finais carregadas com momento transversal $p_{T}>1.0 \mathrm{GeV}$, obtém-se o resultado mostrado na parte (B) dessa figura, chegando-se a uma fração de $0.7 \%$ dos eventos sobrevivendo com um gap nos casos de fusão de W's.

- Já com o corte em $p_{T}>2.0 \mathrm{GeV}$ mostrado na parte (C) da figura, chega-se a aproximadamente $8 \%$ dos gaps sobrevivendo para um higgs produzido por fusão de W's com massa $m_{h}>800 \mathrm{GeV}$.

- Para a fusão de gluons, $S$ é sempre muito pequena, embora seja nula somente na parte (A) da figura.

- Mantém-se, como no caso anterior, o crescimento da probabilidade de sobrevivência do gap com a massa do higgs para a fusão de W's.

Devido a essa probabilidade de sobrevivência do gap muito pequena, foram tentados os outros grupos de cortes, Grupo II e Grupo III.

Os resultados referentes ao grupo de cortes II são apresentados nas fig. (7.3) para o intervalo de rapidez $-3<y<3$, sem interações múltiplas. Os itens (A), (B) e (C) têm o mesmo significado que para as figuras anteriores, e que se manterá para todas as posteriores:

(A) Fração de eventos em que o intervalo de rapidez considerado é mantido vazio, ou seja, não é atingido por nenhuma partícula carregada. Os muons e as partículas neutras não estão sendo levadas em consideração. 


\section{PYTHIA GrupoI (c/IM)}
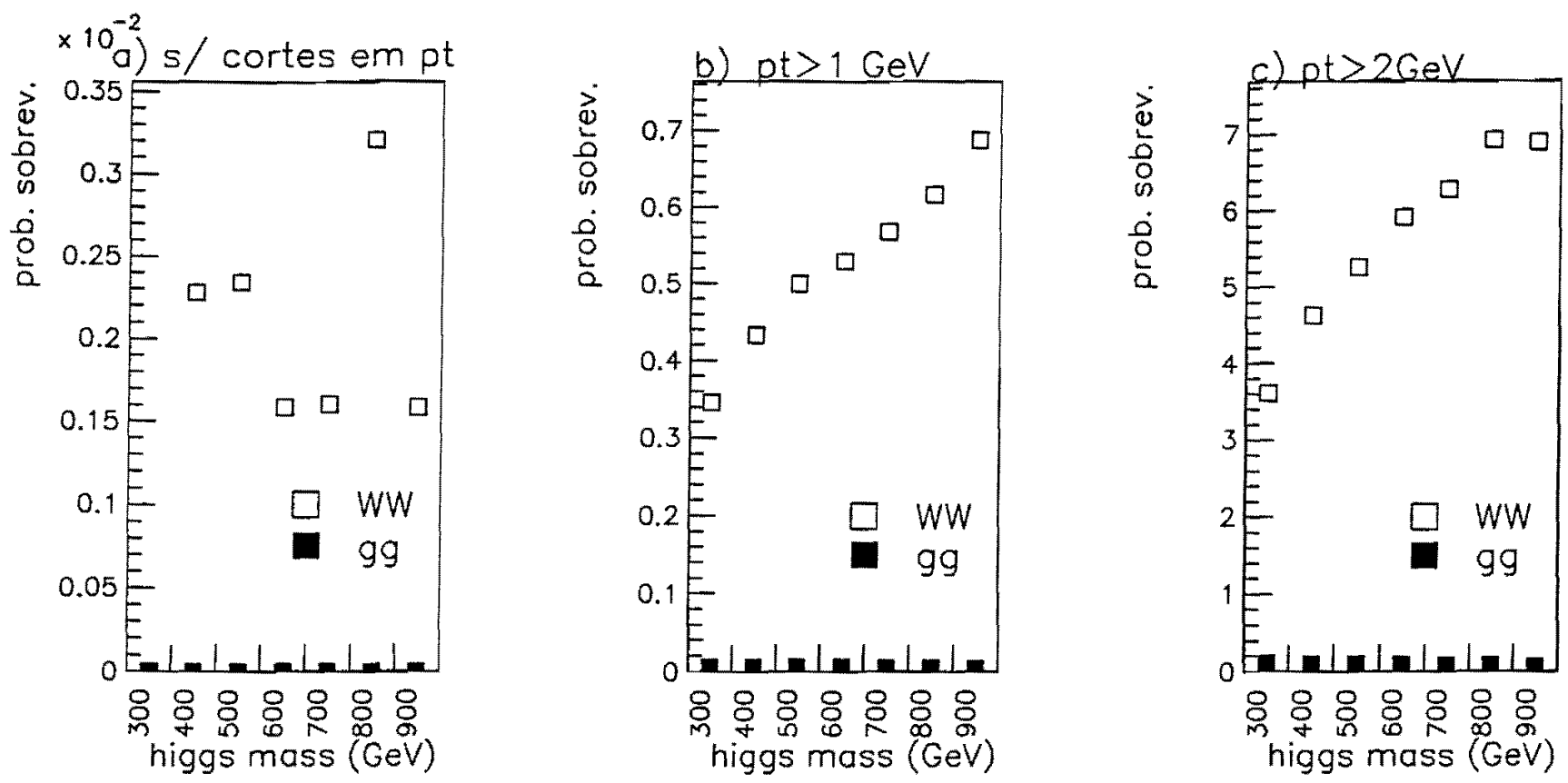

Figura 7.2: Probabilidade de Sobrevivência do Gap na formação de um higgs por fusão de W's e de gluons. As situações (A), (B) e (C) estão descritas na pag. (104).IM=Interações Múltiplas. 


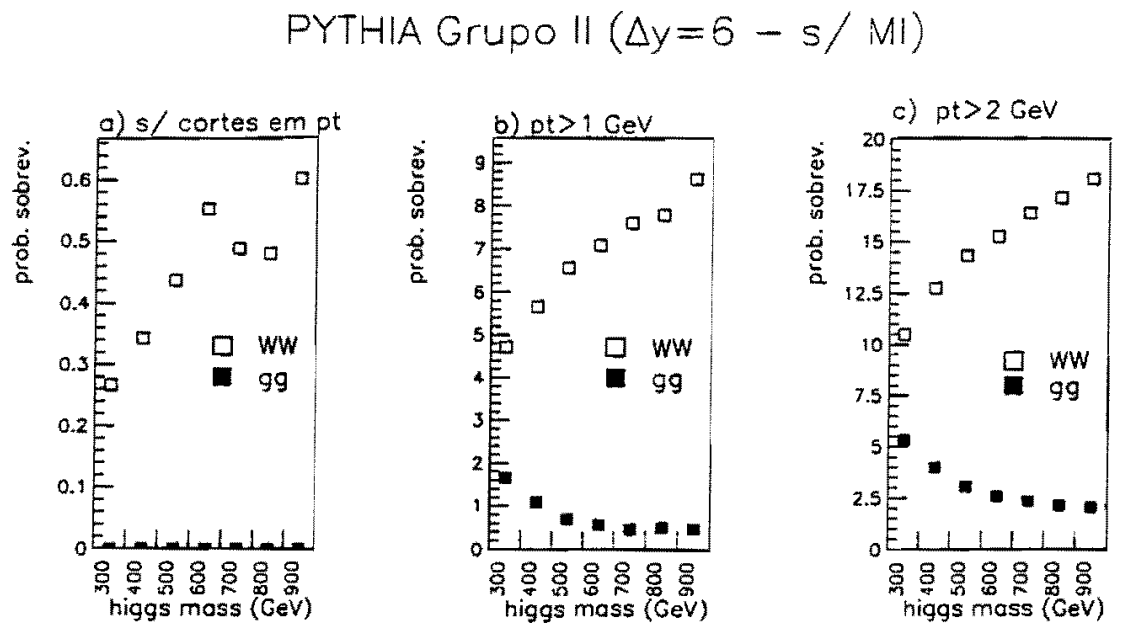

Figura 7.3: Probabilidade de Sobrevivência do Gap na formação de um higgs por fusão de W's e de gluons. As situações (A), (B) e (C) estão descritas na pag.(107).IM=Interações Múltiplas.

(B) Fração de eventos em que o intervalo de rapidez considerado é mantido vazio quando são consideradas somente as partículas carregadas com $p_{t}>1.0 \mathrm{GeV}$. Os muons, as partículas neutras e as partículas carregadas com momento transversal $p_{T}<1.0 \mathrm{GeV}$ não estão sendo levadas em consideração.

(C) Fração de eventos em que o intervalo de rapidez considerado é mantido vazio quando são consideradas somente as partículas carregadas com $p_{t}>2.0 \mathrm{GeV}$. Os muons, as partículas neutras e as partículas carregadas com momento transversal $p_{T}<2.0 \mathrm{GeV}$ não estão sendo levados em consideração.

Verifica-se na fig.( 7.3) que:

- A probabilidade de sobrevivência do gap obtida com os cortes do Grupo II para a fusão de W's, tem valores semelhantes aos obtidos na fig.( 7.1), onde eram aplicados os cortes do Grupo I quando se inclui todas as partículas carregadas (menos os muons). Quando são aplicados os cortes de momento transversal mínimo das partículas, essa relação se mantém, como pode ser visto nas partes (B) e (C) da fig.( 7.3). 
- Observa-se ao mesmo tempo, que nos dois últimos casos ((B) e (C)), a fração de eventos com fusão de gluons que sobrevive aos cortes também aumenta, embora ainda seja menor que para a fusão de W's.

- O comportamento de $S$ em função da massa do higgs é para a fusão de W's é oposto àquele para a fusão de gluons: $S$ cresce com $m_{h}$ no primeiro caso, e decresce no segundo.

- Além disso, deve-se ter em mente que uma fração de eventos maior sobrevive aos cortes do Grupo II que aos cortes do Grupo I.

A diminuição do intervalo de rapidez vai propiciar resultados ainda melhores, conforme pode ser observado na fig.(7.4), para $\Delta y=4$ :

- Quando são considerados todos os hadrons finais carregados, parte (A), já se obtém um bom resultado para a fusão de W's, com $S$ variando entre aproximadamente $4 \%$ e $8 \%$, enquanto para a fusão de gluons, essa grandeza permanece nula para todas as massas do higgs.

- Quando são introduzidos os cortes nos momentos transversais dos hadrons finais, partes (B) e (C) da figura, $S$ aumenta bastante para a produção de higgs por fusão de W's, chegando quase a $50 \%$ quando o higgs tem massa de $900 \mathrm{GeV}$, e tendo sido eliminadas as partículas com momento transversal menor que $2.0 \mathrm{GeV}$. Mesmo para massas menores, como $m_{h}=300 \mathrm{GeV}$, obtém-se $S=30 \%$, que é um resultado excelente.

- A probabilidade de sobrevivência do gap para os processos de produção de higgs por fusão de gluons também aumenta bastante, chegando a mais de $10 \%$ quando se considera um higgs de massa $m_{h}=300 \mathrm{GeV}$, em eventos em que são cortados os hadrons finais com $p_{T}<2.0 \mathrm{GeV}$. Porém, esses valores de $S$ não chegam a atingir, na faixa de massa estudada, os valores obtidos para a fusão de W's.

- A melhora dos resultados com relação ao intervalo de rapidez considerado anteriormente, que era maior, se deve a dois motivos:

1. Está sendo procurado um gap menor, e que portanto, tem maior probabilidade de sobrevivência. 


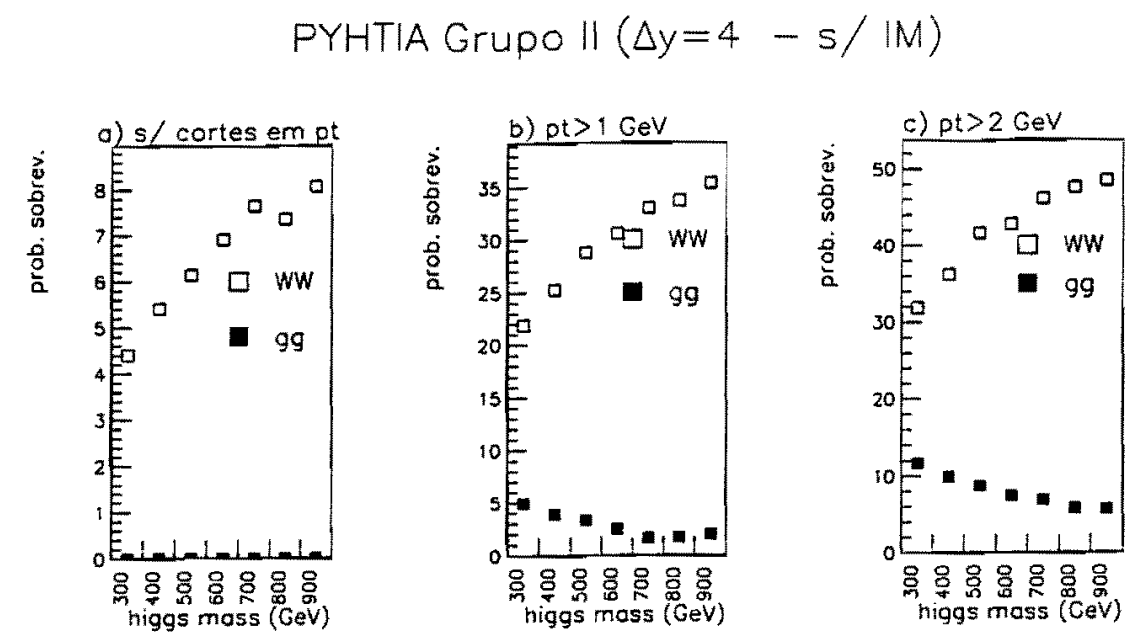

Figura 7.4: Probabilidade de Sobrevivência do Gap na formação de um higgs por fusão de W's e de gluons. As situações (A), (B) e (C) estão descritas na pag.(107). IM=Interações Múltiplas.

2. Foram aceitos eventos que tivessem pelo menos um jato com rapidez no intervalo $2<y<5$, de forma que grande parte dos eventos já tem o intervalo onde se procura o gap contaminado a priori. Porém, é interessante conhecer a fração de eventos que possui um gap de 6 unidades de rapidez, centrado em zero, sem contaminação. É muito pouco provável que um evento proveniente do fundo apresente um gap desse tamanho.

As fig. ( 7.6) e ( 7.5) representam a probabilidade de sobrevivência do gap para produção de higgs por fusão de W's e de gluons, usando o Grupo II de cortes, e com as interações múltiplas sendo levadas em consideração, respectivamente para os casos em que se toma um intervalo de rapidez $\Delta y=6.0 \mathrm{e}$ outro de $\Delta y=4.0$, ambos centrados em zero.

As conclusões a que se chega a partir da observação das figuras são:

- Se não for feito nenhum corte no momento transversal dos hadrons finais, a probabilidade de sobrevivência do gap só não é nula, embora 


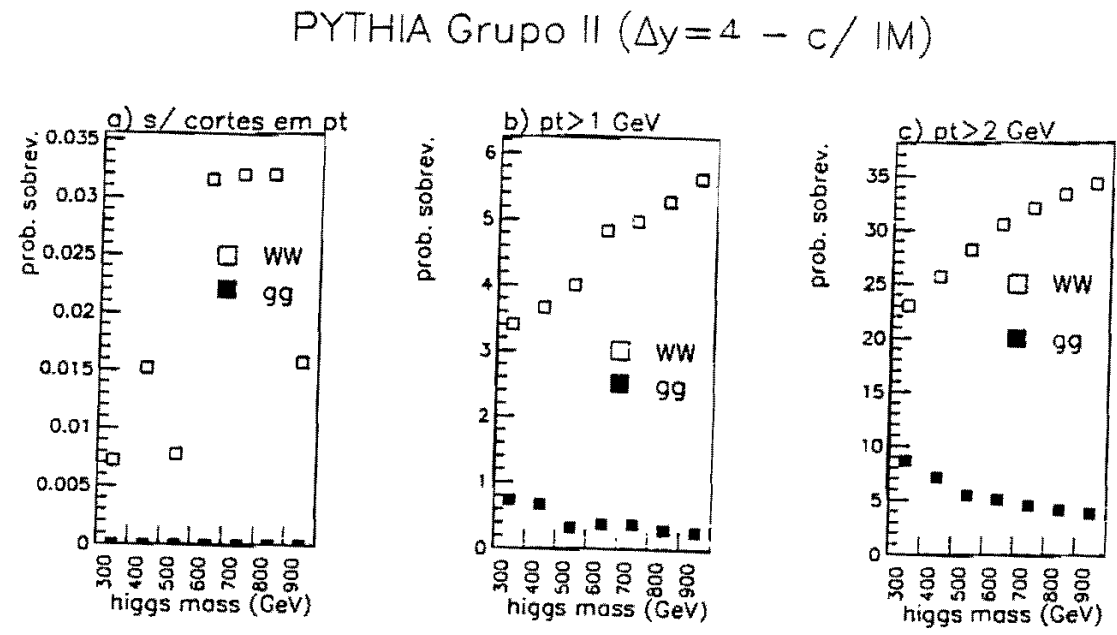

Figura 7.5: Probabilidade de Sobrevivência do Gap na formação de um higgs por fusão de W's e de gluons. As situações (A), (B) e (C) estão descritas na pag.(107). IM=Interações Múltiplas.

PYTHIA Grupo $\|(\Delta y=6-c / \mid M)$
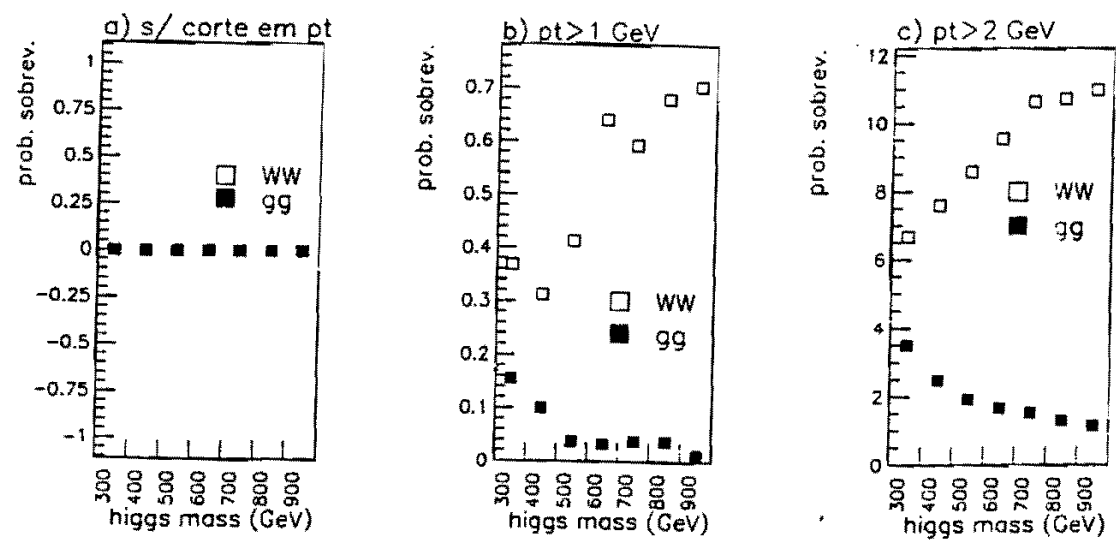

Figura 7.6: Probabilidade de Sobrevivência do Gap na formação de um higgs por fusão de W's e de gluons. As situações (A), (B) e (C) estão descritas na pag.(107). IM=Interações Múltiplas. 
seja ainda muito pequena, para a fusão de W's quando é considerado o intervalo menor de rapidez, $\Delta y=4.0$.

- Considerando-se os cortes já discutidos de $p_{T}$, os resultados obtidos são extremamente animadores, sendo que a parte (C) da fig. (7.5) apresenta uma alta fração dos eventos em que o gap de rapidez sobrevive.

- A probabilidade de sobrevivência do gap para a fusão de gluons também aumenta quando se fazem os cortes em $p_{T}$, mas não chegam aos valores atingidos pela fusão de W's, embora para pequenas massas do higgs, os valores se aproximem bastante, como se observa na parte (C) da figura.

- Mais uma vez, observa-se o comportamento inverso de $S$ em função de $m_{h}$ para a fusão de gluons (decrescente) e para a fusão de W's (crescente).

Há ainda o terceiro grupo de cortes a ser analisado. Nesse caso também foram considerados dois intervalos de rapidez: $-3.0<y<3.0$ e $-2.0<y<$ 2.0, mostrados respectivamente nas fig. (7.8) e fig. (7.7). As observações a serem feitas sobre esses resultados, obtidos sem a incorporação das interações múltiplas são:

- Como era de se esperar, os resultados são melhores para $\Delta y=4$ do que para $\Delta y=6$, porque se está procurando um gap menor, que portanto tem maior probabilidade de sobrevivência.

- Considerando-se os itens (B) e (C) das duas figuras, conclui-se que eliminando-se as partículas com $p_{T}$ muito pequeno, a probabilidade de sobrevivência do gap é grande, ultrapassando $40 \%$ dos eventos quando se considera o intervalo menor de rapidez.

A inclusão das interações múltiplas não atrapalha demais esses resultados, como é mostrado nas fig. (7.9) e (7.10):

- No item (A) das duas figuras, onde são incluídos todos os hadrons finais, a probabilidade de sobrevivência do gap é nula, ou muito pequena.

- Repetem-se os comportamentos observados anteriormente de $S$ crescer com $m_{h}$ para os processos de fusão de W's e decrescer para os processos de fusão de gluons. 


$$
\text { PYTHIA Grupo III }(\Delta y=4-s / \mid M)
$$
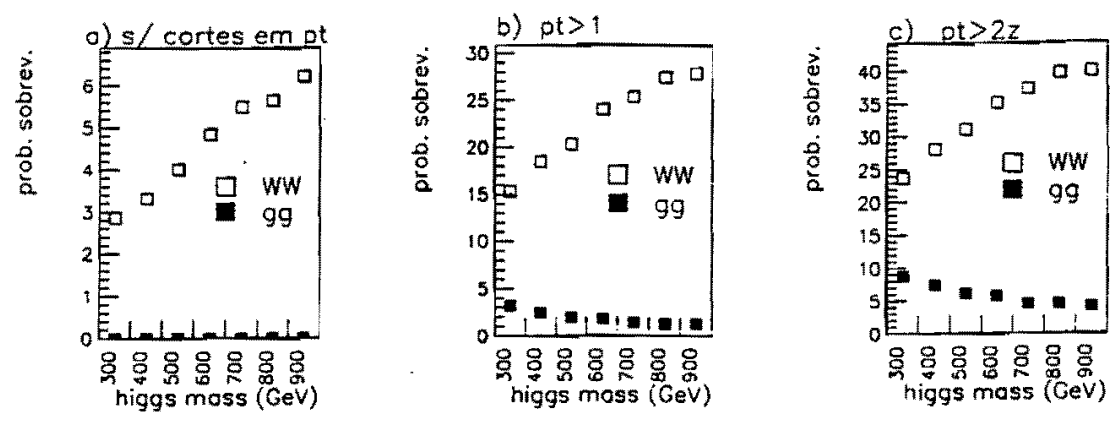

Figura 7.7: Probabilidade de Sobrevivência do Gap na formação de um higgs por fusão de W's e de gluons. As situações (A), (B) e (C) estão descritas na pag.(107). IM=Interações Múltiplas.

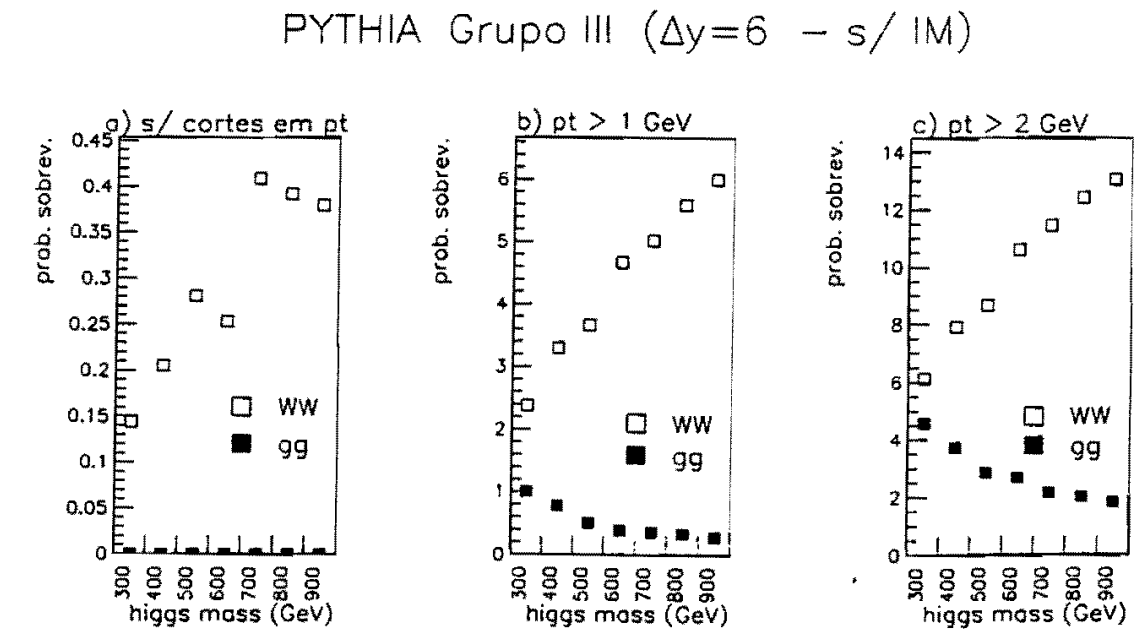

Figura 7.8: Probabilidade de Sobrevivência do Gap na formação de um higgs por fusão de W's e de gluons. As situações (A), (B) e (C) estão descritas na pag.(107). IM=Interações Múltiplas. 
PYTHIA Grupo III $(\Delta y=4 \mathrm{c} / \mathrm{IM})$
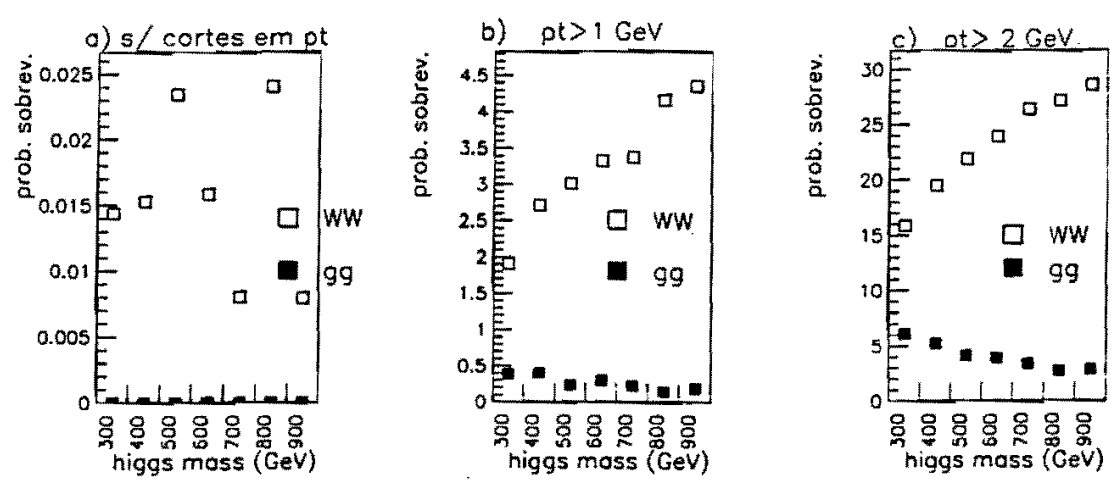

Figura 7.9: Probabilidade de Sobrevivência do Gap na formação de um higgs por fusão de W's e de gluons. As situações (A), (B) e (C) estão descritas na pag.(107). IM=Interações Múltiplas.

- A probabilidade de sobrevivência do gap assume um valor interessante quando é feito o corte eliminando todas as partículas com momento transversal inferior a $2.0 \mathrm{GeV}$, (parte $(\mathrm{C})$ ). Porém, para massas pequenas do higgs, a probabilidade de sobrevivência do gap para a fusão de W's e para a fusão de gluons possuem valores bem próximos, o que tornaria difícil a distinção entre os dois tipos de processos.

\subsection{Seção de Choque e Luminosidade}

Pelo que foi apresentado e discutido anteriormente, seria possível chegar-se a algumas conclusões:

- Desde que sejam feitos cortes no momento transversal dos hadrons finais, a probabilidade de sobrevivência do gap é apreciável para massas do higgs grandes, e em muitos casos, mesmo para as massas menores tem um valor que pode ser considerado satisfatório. 


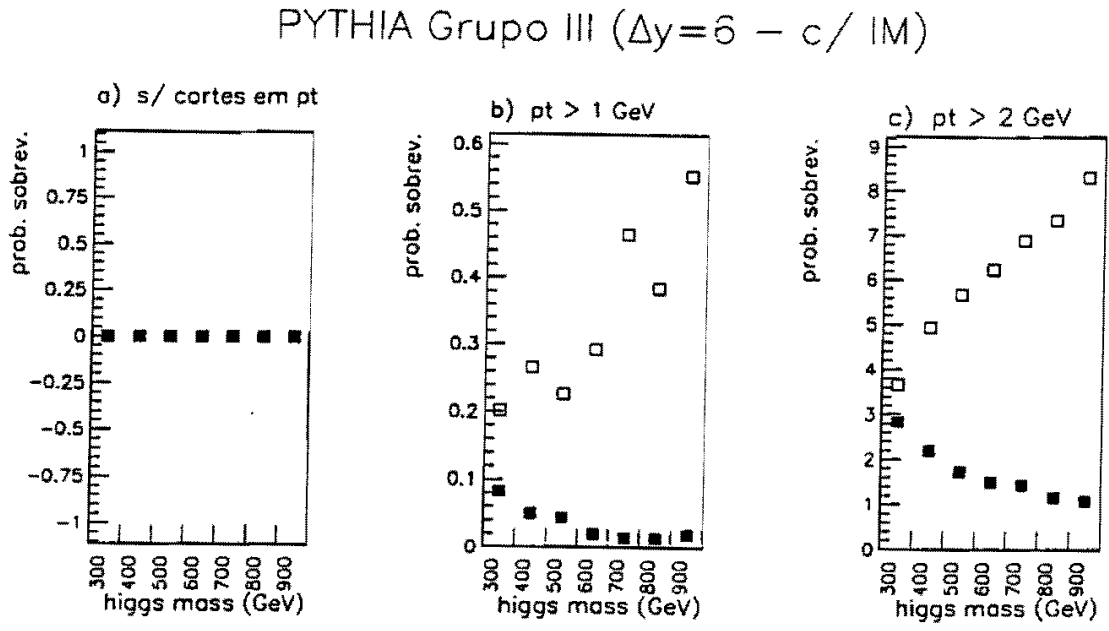

Figura 7.10: Probabilidade de Sobrevivência do Gap na formação de um higgs por fusão de W's e de gluons. As situações (A), (B) e (C) estão descritas na pag. (107). IM=Interações Múltiplas.

- Porém, a razão entre as probabilidade de sobrevivência do gap para os dois processos diminui quando se aumenta o valor do corte em $p_{t}$ pois este corte aumenta o valor de $S$ para a fusão de W's, causando efeito equivalente, porém não tão marcante na fusão de gluons. Para pequenas massas do higgs, esse comportamento da fusão de gluons deve comprometer gravemente o uso de $S$ (ver fig. (7.8) e ( 7.7).

- A inclusão das interações múltiplas modifica o resultado numérico dessas conclusões, porém não invalida a análise qualitativa: com um corte no momento transversal dos hadrons finais, é possivel, conforme o grupo de cortes adotado para o reconhecimento do evento, obter grandes valores de $S$, ou seja, uma alta taxa de sobrevivência do gap de rapidez.

- O grupo de cortes que produz melhor resultado em termos de obtenção de eventos com gap depende da massa do higgs. Para massas pequenas, o grupo I é o que dá a maior taxa $W W_{\text {gap }} / g g_{g a p}$, onde $W W_{\text {gap }}$ representa o número de eventos gerados por fusão de W's em que o gap sobrevive, e $g g_{\text {gap }}$ tem o mesmo significado para a fusão de gluons. Para massas grandes, todos os grupos obtêm para essa mesma taxa um valor $\sim 10$, 
que é muito boa. Mas com o grupo II o número de eventos será maior, e por isso talvez ele deva ser preferido.

Considerando-se que um dos objetivos da tese é analisar a possibilidade de se distinguir entre um higgs produzido pela fusão de gluons de outro produzido pela fusão de bosons vertoriais $\mathrm{W}$, é preciso lembrar que em cada um dos gráficos, os grupos de eventos submetidos à analise deste trabalho foi previamente submetido ao mesmo grupo de cortes, e são, portanto, indistinguíveis do ponto de vista do experimento. Dessa forma, um resultado como o apresentado na parte (C) da fig. (7.7) indica que, numa experiência real, se for encontrado um evento com um higgs de massa próxima de 300 $\mathrm{GeV}$, e que após um corte de momento transversal dos hadrons finais apresentou um gap, ele pode ter sido produzido tanto por uma fusão de gluons como por uma fusão de W's. E a variável $S$ nem sequer dá uma indicação de qual dos dois processos tem maior probabilidade de haver ocorrido. Isso porque estão faltando dois ingredientes para essa análise, que são a seção de choque dos processos e a luminosidade com que trabalhará o acelerador, e que permitirão avaliar o número de eventos de cada tipo que poderá ser esperado por ano. A introdução desses dois fatores não ajudará na distinção entre os dois processos, mas permitirá determinar se é possível ou não distingui-los experimentalmente.

As figuras a seguir foram produzidas de forma ligeiramente diferente das apresentadas anteriormente: $o$ valor da probabilidade de sobrevivência do gap que havia sido mostrado, é multiplicado pela seção de choque do processo e multiplicado por uma luminosidade, que foi tomada como sendo a do LHC, $\mathcal{L}=10^{34} \mathrm{~cm}^{-2} \mathrm{~s}^{-1} \simeq 100$ eventos/fb-ano, obtendo-se assim, o número de eventos por ano que é gerado nesse acelerador.

A disposição dessas novas figuras será um pouco modificada com relação às anteriores. Cada figura consta de seis partes, sendo que as três superiores apresentam o resultado sem levar em consideração a possibilidade de interações múltiplas, e as três inferiores levam essa possibilidade em consideração. Embora em termos experimentais seja mais razoável considerar sempre a possibilidade de interações múltiplas, que de fato devem ocorrer, os resultados estão sendo apresentados com e sem elas porque não há ainda um modelo definitivo para esse tipo de interação, de forma que é necessário conhecer o comportamento do subprocesso principal separadamente. Cada um dos conjuntos de resultados será apresentado nas figuras da seguinte maneira: 
- Sem interações múltiplas:

(A) Todos os hadrons finais são considerados.

(B) São aceitos somente os hadrons finais com $p_{T}>1.0 \mathrm{GeV}$.

(C) São aceitos somente os hadrons finais com $p_{T}>2.0 \mathrm{GeV}$.

- Com interações múltiplas:

(D) Todos os hadrons finais carregados são considerados.

(E) São aceitos somente os hadrons finais carregados com $p_{T}>1.0$ $\mathrm{GeV}$.

(F) São aceitos somente os hadrons finais carregados com $p_{T}>2.0$ $\mathrm{GeV}$.

$\mathrm{Na}$ fig. (7.11), encontram-se os resultados para os eventos que passaram pelos cortes do Grupo I. Algumas conclusões já podem ser tiradas:

- Enquanto a probabilidade de sobrevivência do gap em função da massa do higgs era crescente para a fusão de W's, como foi observado em todas as figuras anteriores, o número de eventos por ano é decrescente, acompanhando o comportamento da seção de choque (que é decrescente).

- O número de eventos por ano em função da massa do higgs para a fusão de gluons também é decrescente.

- Se forem aceitos todos os hadrons finais carregados, como nas partes (A) e (D) da figura, o número de eventos com fusão de gluons que mantém o gap é nulo. Porém deixa de ser quando são desprezadas as partículas com $p_{T}$ muito pequeno, mas ainda assim não são muito animadoras.

- Para fusão de W's e massas grandes do higgs, maiores que 700 ou 800 $\mathrm{GeV}$, espera-se no máximo um evento por ano conservando o gap de rapidez, mesmo quando são cortados os hadrons com pequeno $p_{T}$. 
- Quando são cortados os hadrons com $p_{T}<1.0 \mathrm{GeV}$, somente aqueles casos em que as interações múltiplas não estão ligadas apresentam algum resultado quanto ao gap (sobrevive, por ano, 1 higgs produzido por fusão de W's, e praticamente nenhum por fusão de gluons). Porém essa é certamente uma situação não realista. Levando-se em conta a possibilidade de interações múltiplas, o número de eventos por ano é menor que 1 nos dois casos.

- Quando são eliminados os hadrons com $p_{T}<2.0 \mathrm{GeV}$, chega-se a um número pequeno, em torno de 2 eventos por ano para bosons de Higgs com massa de $300 \mathrm{GeV}$ produzidos por fusão de W's, enquanto quase não existem higgs produzidos por fusão de gluons que sobrevivam aos cortes mantendo um gap sem contaminação.

Na fig. (7.12) são apresentados os resultados obtidos com o grupo de cortes II, para um intervalo de rapidez $\Delta y=4.0$. Observa-se que:

- Mantém-se o comportamento decrescente do número de eventos por ano em função da massa do higgs, tanto para fusão de W's como de gluons, que foi observado na fig. (7.11), e que no caso da fusão de W's é um comportamento oposto ao da probabilidade de sobrevivência do gap.

- Quando nenhuma das partículas finais é cortada, e não são consideradas interações múltiplas, há uma chance pequena de se observar um evento por ano com gap de rapidez na fusão de W's, enquanto a fusão de gluons não produziria nenhum evento limpo. Porém, como é de se esperar, quando as interações múltiplas são introduzidas, praticamente nenhum evento é visto, não importa de que tipo.

- Fazendo-se um corte que aceite somente os hadrons com $p_{T}>1.0$ $\mathrm{GeV}$, para massas pequenas do higgs, espera-se de 2 a 3 higgses por ano produzidos por fusão de W's, mas em contrapartida aparecem 1 ou 2 eventos produzidos por fusão de gluons. Se forem introduzidas as interações múltiplas, a razão entre os eventos com gap produzidos por fusão de W's e por fusão de gluons se mantém praticamente a mesma, porém os números absolutos são muito menores, e a observação do evento fica difícil (praticamente impossível). 

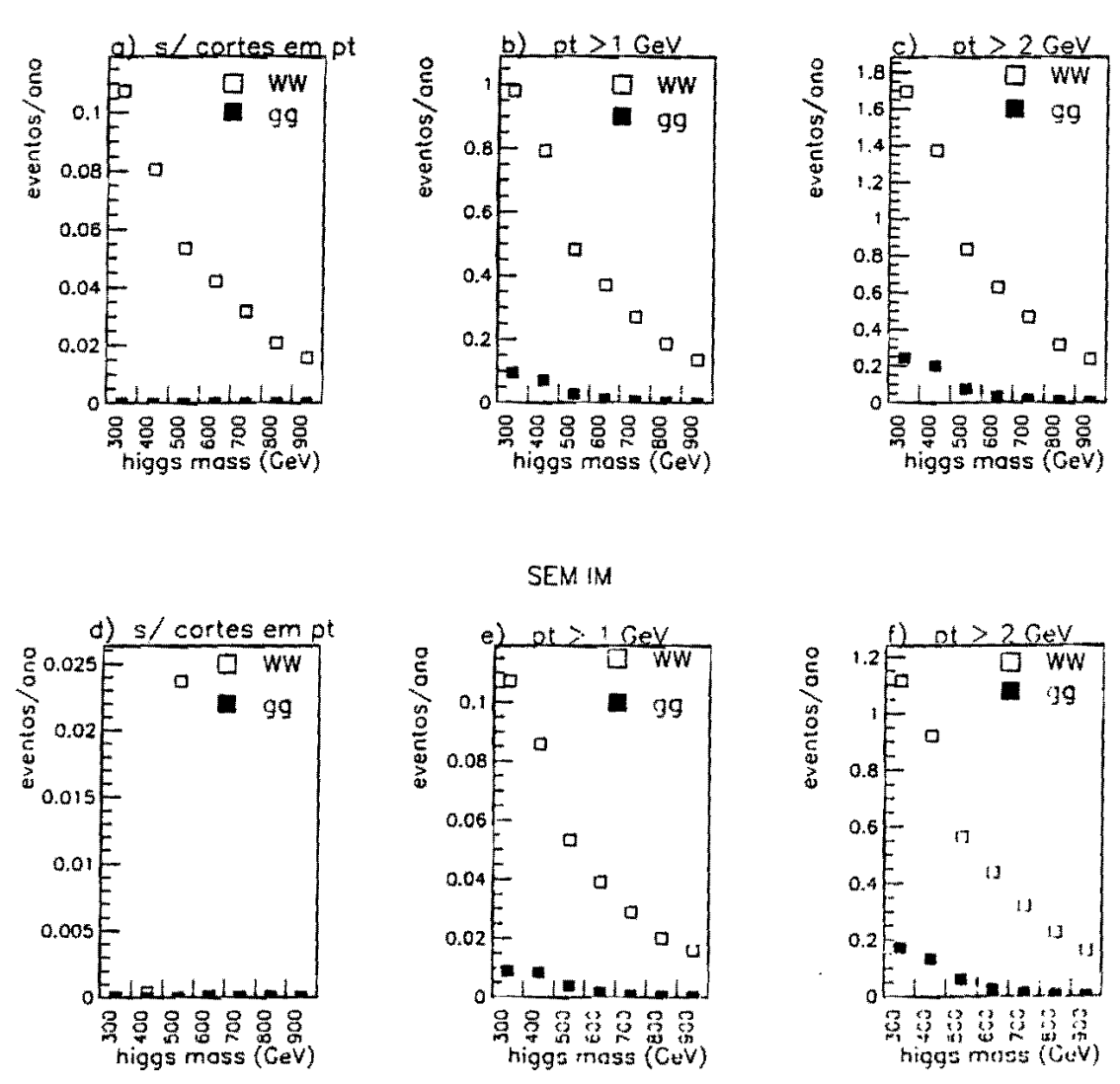

Figura 7.11: Número de eventos contendo gaps produzidos por ano na formação de um higgs por fusão de W's e de gluons. O tipo de processo incluído em cada item da figura está descrito na pag. (117). IM=Interações Múltiplas. 
- Se o corte for feito para aceitar os hadrons com $p_{T}>2.0 \mathrm{GeV}$, novamente aumenta o número de eventos por ano que mantêmo gap, mesmo quando as interações múltiplas estão ligadas, chegando-se a obter quase 3 eventos com gap na fusão de W's para um higgs com $m_{h}=300 \mathrm{GeV}$. Mas, os eventos com fusão de gluons também conservam o gap, e para esse mesmo valor de massa do higgs, são esperados 2 eventos com gap produzidos pela fusão de gluons, o que torna inviável querer distinguir os dois processos de produção do higgs por esse meio. Além disso, para $m_{h}=500 \mathrm{GeV}$, é praticamente 1 evento por ano que pode ser observado.

A fig. (7.13) mostra os resultados para número de eventos por ano em funcão da masșa do higgs, quando são usados os cortes do Grupo III, e é considerado um intervalo de rapidez $\Delta y=4.0$. De sua observação, concluise que:

- Mantém-se o fato de que o número de eventos por ano em função da massa do higgs é decrescente para os dois processos de produção estudados.

- São encontrados alguns eventos com gap quando se considera todos os hadrons finais (partes (A) e (D) da figura), produzidos por fusão de W's para higgses com massas pequenas. Mas mais uma vez, a introdução de interações múltiplas leva ao desaparecimento do gap em todos os eventos.

- Quando é feito o corte no momento transversal dos hadrons finais, aceitando somente os que possuem $p_{T}>1.0 \mathrm{GeV}$, novamente os processos de fusão de gluons são confundidos com os de fusão de W's, pois um número de eventos quase igual dos dois tipos de processo conserva o gap, tanto quando as interações múltiplas estão sendo consideradas, como quando não estão.

- Quando o corte no momento transversal é feito em $2.0 \mathrm{GeV}$, o número de eventos com gap produzido por fusão de gluons supera o número produzido por fusão de W's. Quando as interações múltiplas são consideradas, aproximadamente $1 / 3$ dos eventos são fusões de W's, e os outros $2 / 3$ são fusões de gluons. 
CAPíTULO 7. RESULTADOS E DISCUSSÕES

122

PYTHIA Grupo II $(\Delta y=4)$

SEM IM
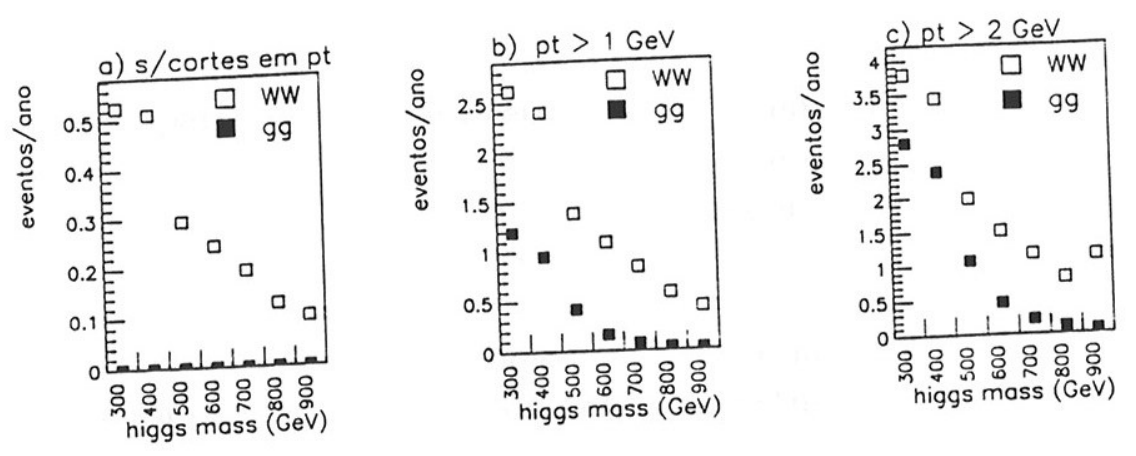

COM IM
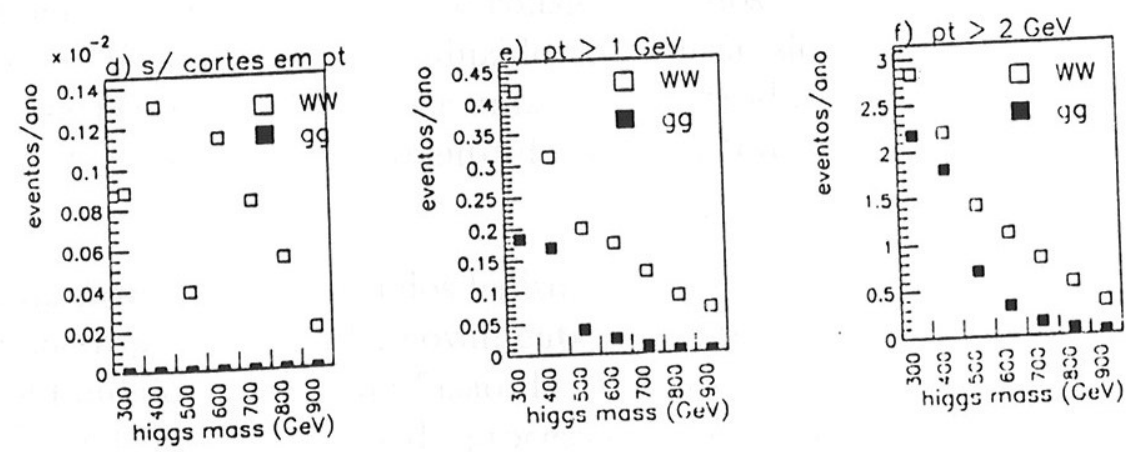

Figura 7.12: Número de eventos contendo gaps produzidos por ano na formação de um higgs por fusão de W's e de gluons. O tipo de processo incluído em cada item da figura está na descrição na pag.(117). IM=Interações Múltiplas. 


\section{PYTHIA Grupo III $(\Delta y=4)$}
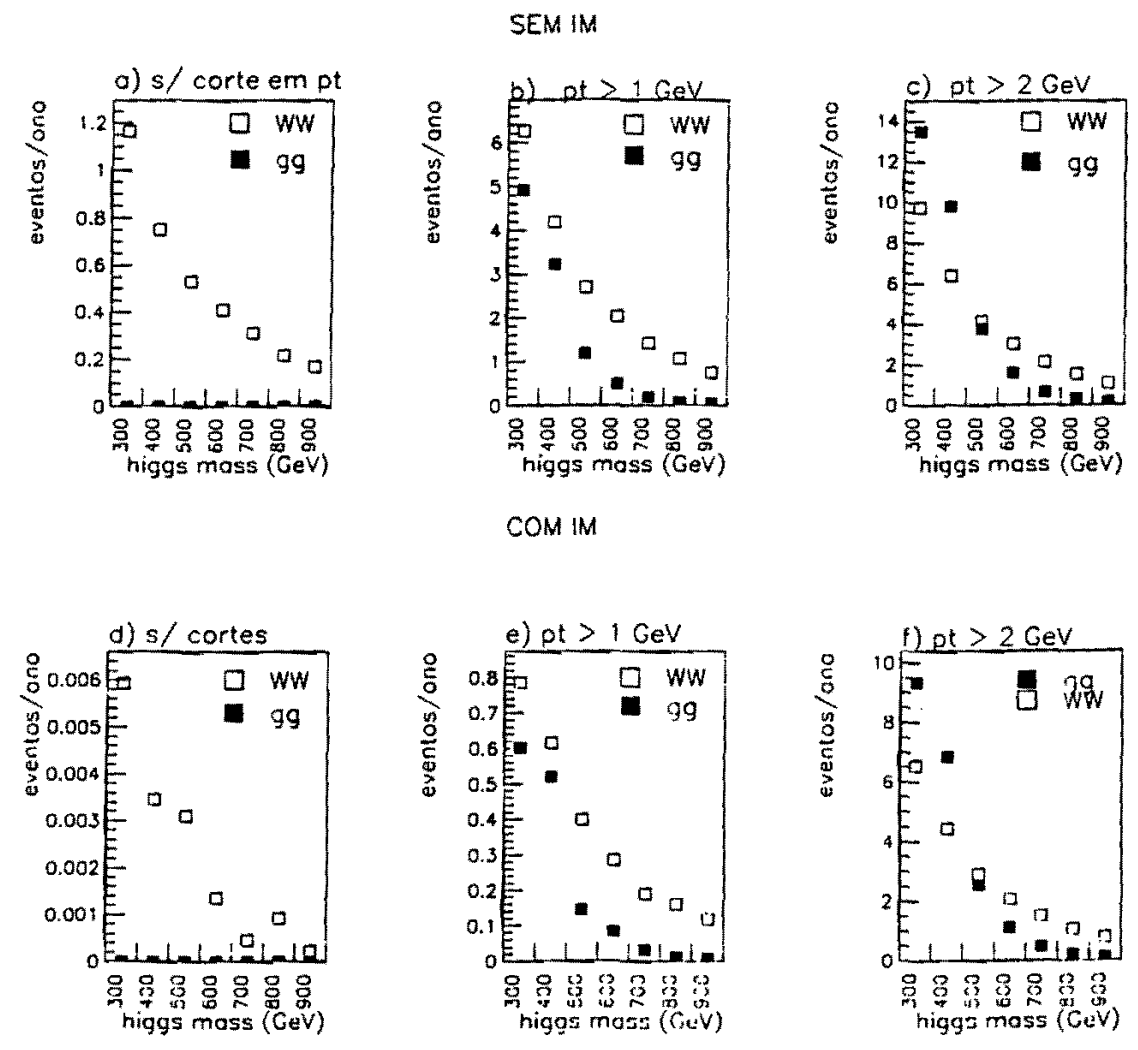

Figura 7.13: Número de eventos contendo gap produzidos por ano na formação de um higgs por fusão de W's e de gluons. O tipo de processo incluído em cada item da figura está na descrição na pag.(117). IM=Interações Múltiplas. 


\section{Primeira Conclusão}

De tudo que foi observado até o momento, algumas conclusões gerais podem ser tiradas:

1. Embora seja extremamente útil, a probabilidade de sobrevivência do gap é uma variável que não dá uma visão completa do que pode ser esperado experimentalmente. Muitas vezes foi encontrada uma probabilidade de sobrevivência do gap muito grande para processos que, devido ao valor da seção de choque, produzirão pouquíssimos ou nenhum evento por ano no LHC, que é na verdade o que interessa no momento.

2. O número de eventos por ano é uma variável mais prática, que permite visualizar melhor que pode ser esperado experimentalmente.

3. Em geral a fusão de W's apresenta um número maior de eventos com gap do que a fusão de gluons. A exceção fica por conta dos resultados apresentados na fig (7.13) onde, após os cortes em pt, a fusão de gluons confunde-se ou chega a superar a fusão de W's.

4. Ao contrário do que ocorre para a probabilidade de sobrevivência do gap, que para fusão de W's aumenta com $m_{h}$, o número de eventos por ano decresce para $m_{h}$ crescente. Já para a fusão de gluons, as duas grandezas apresentam o mesmo comportamento decrescente com $m_{h}$.

5. Se os resultados forem analisados cruamente, sem a imposição de cortes, é impossível usar o gap como assinatura. A melhor de todas as situações ocorre na parte (A) da fig. (7.13), que mostra ser possível achar um, ou talvez até dois eventos num ano com gap, quando o evento é tratado sozinho, ou seja, sem interações múltiplas. Porém, introduzindo as interações múltiplas já não há possibilidade nenhuma de encontrar um evento cujo gap não tenha sido contaminado.

6. Os cortes em pt, eliminando as partículas com pequeno momento transversal facilitam a tarefa de encontrar eventos com gap, mas ainda assim persiste a dificuldade. Com um corte razoavelmente drástico, aceitando somente as partículas com $p t>2 \mathrm{GeV}$, consegue-se para alguns dos grupos de cortes resultados positivos, mesmo com inclusão de interações múltiplas(fig. (7.13) e (7.12)). 
7. No entanto quando o número de eventos torna-se apreciável para a fusão de W's, é também apreciável para a fusão de gluons, pois o corte em pt ajuda as duas reações, e o que é pior, ajuda mais a fusão de gluons.

8. Por esse motivo, como se vê nas fig. (7.11), (7.12) e (7.13), não é possível distinguir a fusão de W's da fusão de gluons, quando não há corte em $p_{t}$, porque muito poucos eventos subsistem com gap, e quando se impõe o corte em $p_{t}$, vários eventos são encontrados com gap, mas o mesmo ocorre com o processo de fundo, a fusão de gluons.

\subsection{Comparando Dois Modelos de Minimum Bias}

O modelo para interações múltiplas utilizado pelo PYTHIA foi comparado com outro modelo para interações nos estados inicial e final, usado pelo DTUJET. O DTUJET não possui processos eletrofracos, e a maneira encontrada de produzir os eventos com esse programa está descrita na pag. (94).

A fig. (7.15) mostra a probabilidade de sobrevivência do gap para fusão de bosons W obtida para o DTUJET e para o PYTHIA (foram usados os cortes do Grupo III, e incluídas as interações múltiplas; os resultados para o PYTHIA são os mesmos apresentados nas figuras (7.9) e (7.10)). Na parte superior da figura estão os resultados para intervalos de rapidez com largura $\Delta y=6$, sendo aceitos todos os hadrons finais carregados na parte (A) da figura, os hadrons finais carregados com $p_{T}>1.0 \mathrm{GeV}$ na parte (B), e os hadrons finais carregados com $p_{T}>2.0 \mathrm{GeV}$ na parte (C). Na parte inferior, estão dispostos os resultados para intervalos de rapidez com largura $\Delta y=4$, novamente para todos os hadrons finais (D), hadrons finais com $p_{T}>1.0$ (E), e hadrons finais com $p_{T}>2.0(\mathrm{~F})$.

Algumas conclusões podem ser prontamente tiradas desse conjunto de figuras:

- Os resultados obtidos com o DTUJET quando não é feito nenhum corte no momento transversal dos hadrons finais (partes (A) e (D) da figura), não tem significado estatístico pois, para todas as massas consideradas, muito poucos eventos passaram por todos os cortes (cerca de 5 eventos, ou em muitos casos, menos). 


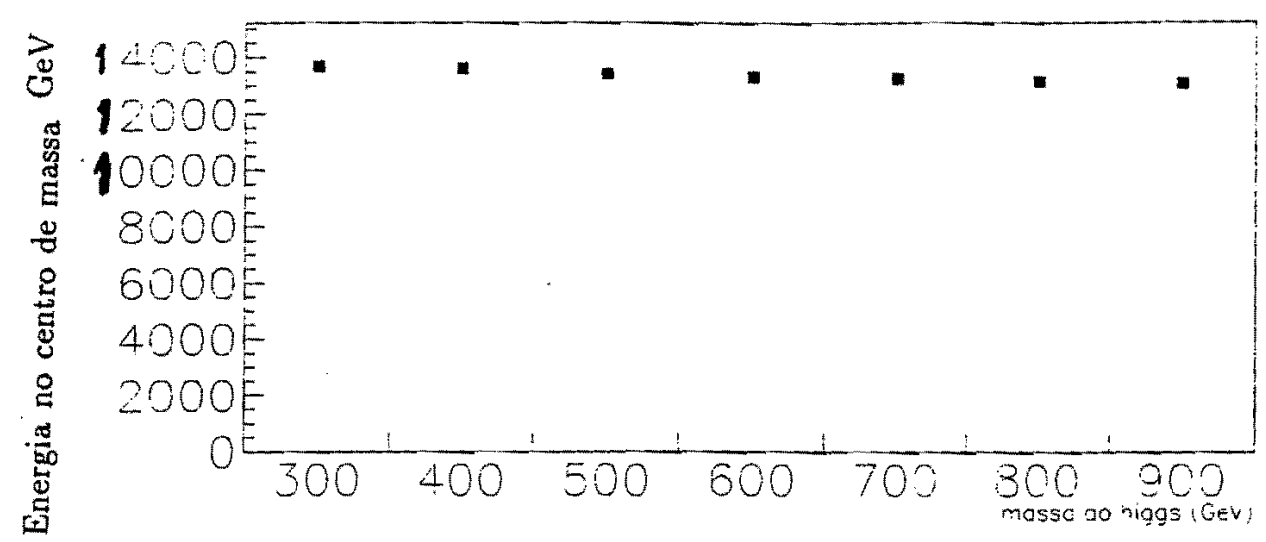

Figura 7.14: Energia no centro de massa utilizada em função da massa do higgs para processos rodados com o DTUJET (vide pag.(94)).

- Os resultados obtidos com o DTUJET são ligeiramente crescentes para pequenas massas do higgs, mas logo atingem um patamar, permanecendo a partir de entâo constantes. Este é um resultado esperado pois, em cada um dos gráficos, a única variável que está sendo alterada nos pontos obtidos com o DTUJET é a energia no centro de massa, e essa variação não é muito grande, conforme se verifica na fig. (7.14).

- Em todos os casos, o DTUJET produziu muito mais eventos com gap que o PYTHIA. A diferença, embora ainda marcante, é menor quando são considerados intervalos de rapidez $\Delta \eta=4.0$, e são aceitos os hadrons finais com $p_{T}>2 . \mathrm{GeV}$ (parte (F)).

A fig. (7.16) apresenta o número de eventos por ano produzido em cada um dos seis casos analisados na fig. (7.15). Observa-se que:

- Tanto para o PYTHIA como para o DTUJET o número de eventos por ano é decrescente para massas de higgs crescentes. Como anteriormente, isso reflete o fato da seção de choque ser decrescente com a massa do higgs.

- Para o DTUJET há um número de eventos por ano muito maior que para o PYTHIA (nenhum resultado do PYTHIA é nulo, embora pareça 
PYTHIA (GRUPO III) - DTUJET (ambos com IM)

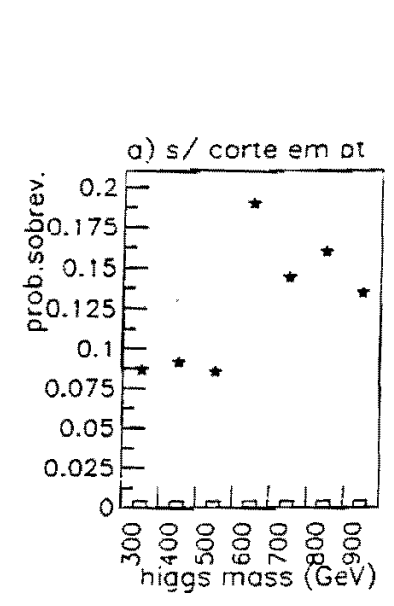

$\Delta \eta=6$

$\star$ dtuje:
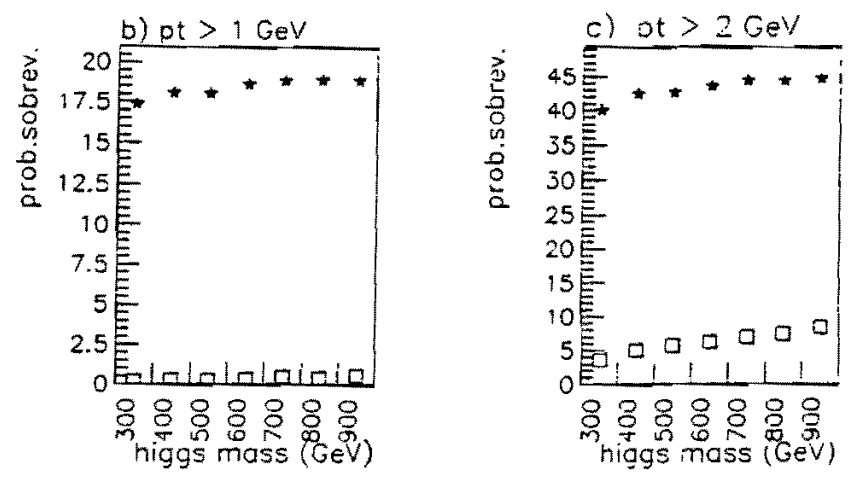

$\Delta \eta=4$

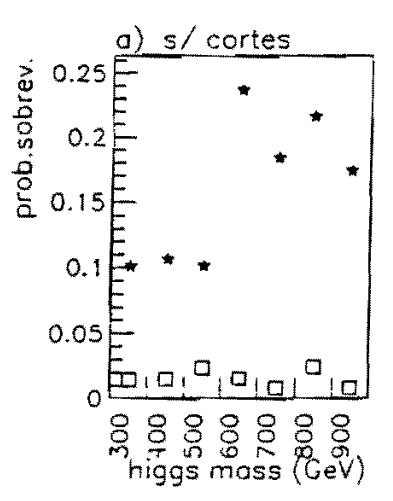

b) pt $>1 \mathrm{GeV}$

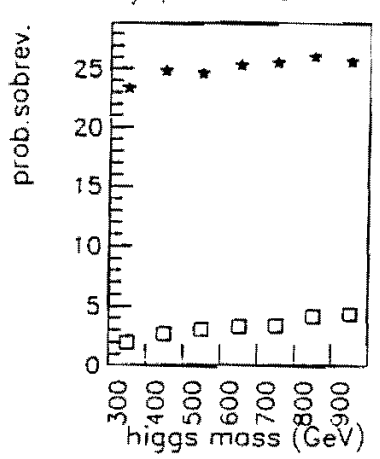

c) pt $>2 \mathrm{GeV}$

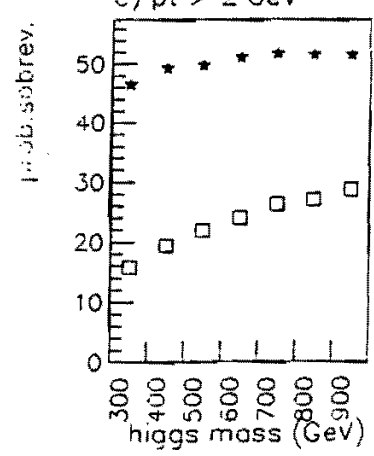

Figura 7.15: Probabilidade de Sobrevivência do Gap para fusão de W's para processos gerados com o PYTHIA(Grupo III e incluindo interações múltiplas) e com o DTUJET. Na pag. (125) há uma explicação sobre as partículas levadas em conta em cada figura. 
no gráfico; eles são muito menores que os valores obtidos com o DTUJET. A diferença é mais acentuada para $m_{h}$ pequena. 
PYTHIA (GRUPO III) - DTUUET (amboscom IM)

$\Delta n=6$
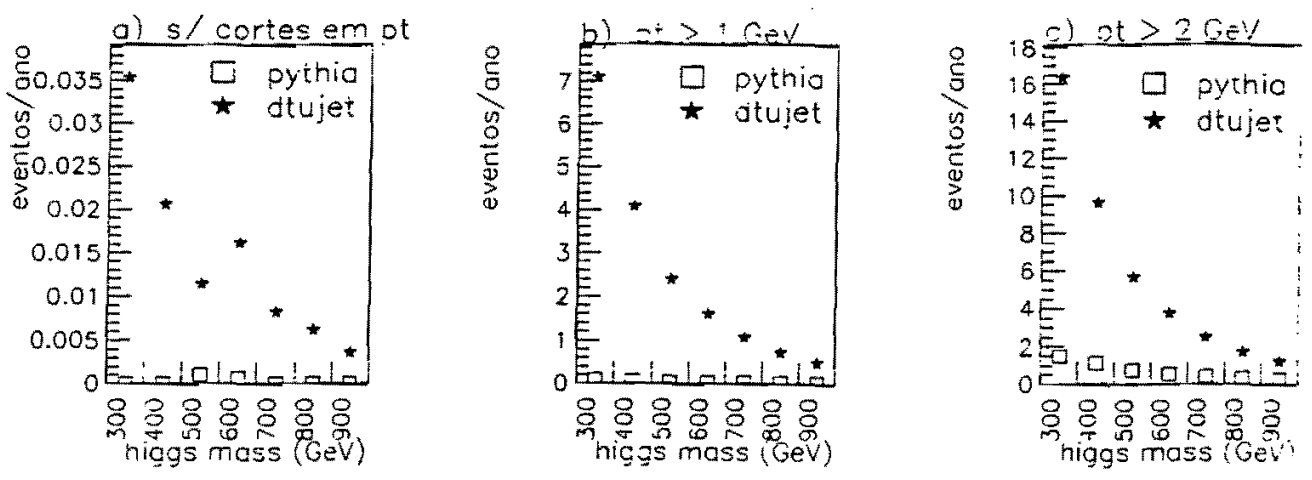

$\Delta \eta=4$
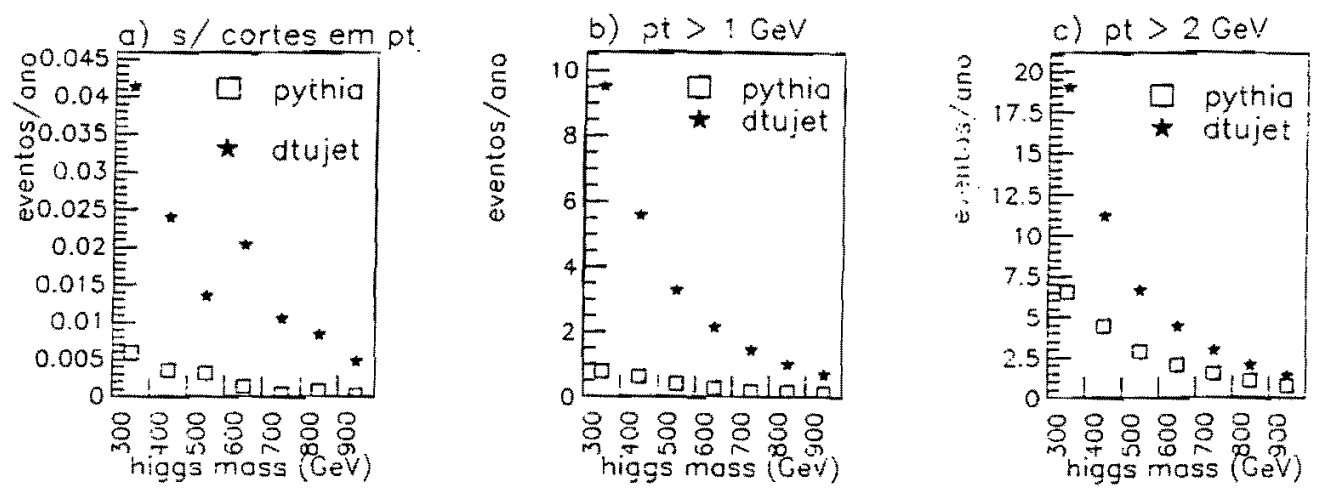

Figura 7.16: número de eventos por ano para fusão de W's para processos gerados com o PYTHIA, usando os cortes do Grupo III (incluindo interações múltiplas) e com o DTUJET. 


\section{Capítulo 8}

\section{Conclusão}

Não é possível usar a existência ou não do gap de rapidez para diferenciar um higgs produzido por fusão de bosons vetoriais de outro produzido por fusão de gluons. A Probabilidade de Sobrevivência do Gap, proposta por Bjorken para calcular a probabilidade de que uma fusão de bosons vetoriais apresente um gap, na verdade fornece a probabilidade de que não ocorram interações múltiplas entre os vários partons dos protons incidentes. Porém, o processo de fragmentação dos partons que restam dos feixes incidentes produz grande número de hadrons e contamina um eventual gap.

Mesmo a modificação dessa definição para uma forma mais apropriada do ponto de vista experimental, dada pelo número de eventos que apresentam gap dividido pelo número total de eventos, apresenta problema pois, conforme foi exaustivamente mostrado ao longo do trabalho, as seções de choque dos processos e a luminosidade do acelerador com que se vai trabalhar são informações essenciais para que se possa verificar a possibilidade de detetar ou não o gap.

As tentativas de evidenciar melhor o gap, eliminando as partículas que tenham chegado ao estado final com pequeno momento transversal não auxiliam, pois a fusão de gluons produz um número maior de partículas, porém mais soft.

Para higgs com massas pequenas, até $\approx 400 \mathrm{GeV}$, é possivel que sejam observados cerca de 10 eventos por ano apresentando gap (fig. 7.13). Desde que não se pretenda separar os higgs produzidos por fusão de W's de outros produzidos por fusão de gluons (o que seria impossível conforme já foi observado), talvez seja possível usar esse sinal para a produção do higgs de forma 
geral, comparando-o por exemplo com o contínuo ZZ.

No que concerne à comparação dos pacotes Monte Carlo, o resultado final foi de certa forma inesperado, pois o DTUJET que sempre efetua troca de pomerons para fazer fragmentação apresenta um número maior de eventos com gap. Por outro lado, esse fato reforça a idéia de que a simulação é necessária para que se tenha uma visão antecipada, ainda que limitada, das previsões experimentais de um modelo.

Há diversos pontos a serem abordados ainda. É possível que num acelerador com maior energia no centro de massa a situação se altere. Seria preciso um trabalho de simulação para esse caso. Um ponto que mereceria ser investigado no futuro é a possibilidade de usar o fato que a fusão de gluons produz minijatos, cujo comportamento seja diferente do comportamento dos hadrons individualmente. 


\section{Bibliografia}

[1] V. Barger e R. J. N. Phillips, in Collider Physics, ed. Addison-Wesley Publishing Company, série Frontiers in Physics, vol. 71, 1990.

[2] S. L. Glashow, Nucl. Phys., 22 (1961) 579.

[3] S. Weinberg Phys. Rev. Lett. 19 (1967) 1264.

[4] A. Salam in Proc. $8^{\text {th }}$ Nobel Symp., ed. N. Svartholm, p.367, Stockholm: Almqvist \& Wiksells (1968).

[5] P. W. Higgs, Phys. Lett. 12 (1964) 132;

Phys Rev. Lett. 13 (1964) 508;

Phys. Lett. 145 (1966) 1156.

[6] F. Englert, R. Brout, Phys Rev. Lett. 13 (1967) 321.

[7] T. Kibble, Phys. Rev. 155 (1967) 1554.

[8] M. Kobayashi e T. Maskawa, Prog. Theor. Phys. 49 (1973) 652.

N. Cabibbo, Phys. Rev. Lett. 10 (1963) 531.

[9] G. 't Hooft, Nucl. Phys. B35 (1971) 167.

[10] B. W. Lee e J. Zinn-Justin, Phys. Rev. D5 (1972) 3121, 3137, 3175.

[11] G. 't Hooft e M. Veltman, Nucl. Phys. B44 (1972) 189;

B50 (1972) 318.

[12] J. M. Cornwall, D. N. Levin e G. Tiktopoulos, Phys. Rev. D10 (1974) 1145. 
[13] C. H. Llewwllyn Smith, Phys. Lett. B46 (1973) 233.

[14] B. W. Lee, C. Quigg, H. Tacker, Phys. Rev. D16 (1977) 1519.

[15] M. S. Chanowitz, Ann. Rev. Nuc. Part. Sci. 38 (1988) 323; Proceedings from Higgs Workshop, Erice, Trapani, Itália, julho de 1989.

[16] O. Adriani et al. (L3 Collaboration) preprint CERN PPE/92-128.

[17] H. Veltman, M. Veltman, Acta Polonica B22 (1991) 669.

[18] A. Sirlin, Comm. Nucl. Part. Phys. 21 (1994) 287.

[19] M. Veltman, Acta Polonica B8 (1977) 475.

[20] J. D. Bjorken, in Proceedings of the SLAC Summer Institute on Particle Physics, Stanford California, 1976 editado por M. C. Zipf, SLAC report n. 198, Stanford California, 1977.

[21] E. Gross e P. Yepes, preprint CERN-PPE/92-153.

[22] Phys. Rev. D50 (1995) Particle Data Group.

[23] G. Altarelli, palestra proferida no International Workshop on Electroweak Physics Beyond the Standard Model, Valência, Espanha, outubro de 1991. Preprint CERN TH-6317/91.

[24] G. Bhanot e K. Bitar, Phys. Rev. Lett. 61 (1988) 798.

J. Kuti, L. Lin e Y. Shen, Phys. Rev. Lett. 61 (1988) 678.

M. Gockeler, K. Jansen e T. Neuhaus, Phys. Lett. B273 (1991) 450.

U. M. Heller, H. Neuberger e P. Vranas, Phys. Lett. B283 (1992) 355.

[25] A. D. Linde, JETP Lett. 23 (1976) 64;

Phys. Lett. B62 (1976) 435;

S. Weinberg, Phys. Rev. Lett. 36 (1976) 294.

[26] A. I. Vainshtein, M. B. Voloshin, V. I. Zakharov e M. S. Shifman, Sov. J. Nucl.Phys. 30 (1979) 711.

A. I. Vainshtein, V. I. Zakharov e M. A. Shifman, Sov. Phys. Usp. 23 (1980) 429.

M. Voloshin, Sov. J. Nucl. Phys. 44 (1986) 478. 
[27] J. F. Gunion, H. E. Haber, G. L. Kane e S. Dawson, The Higgs Hunters' Guide (Frontiers in Physics, Lecture Note Series), ed. Addison Wesley (1987).

[28] J. Fleischer e F. Jegerlehner, Phys. Rev. D23 (1981) 2001.

[29] S. Dawson e S. Willenbrock, Phys. Lett. B211 (1988) 200.

[30] E. Braaten e J. P. Leveille, Phys. Rev. D22 (1980) 715.

[31] N. Sakai, Phys. Rev. D22 (1980) 2220.

[32] T. Inami e T. Kubota, Nucl. Phys. B179 (1981) 171.

[33] A. Djouadi, palestra proferida no MRST-1984 Meeting What next? Exploring the future of High-Energy Physics, maio de 1994, Montreal, Canadá. Preprint UdeM-GPP-TH-04.

[34] P. Agrawal e S. Ellis, Phys, Lett. B22 (1989) 145.

[35] W. Y. Keung e W. J. Marciano, Phys. Rev. D30 (1984) 248.

[36] B. L. Ioffe e V. Khoze, Sov. J. Nucl. Part. 9 (1978) 50.

[37] J. Ellis, M. Gaillard e D. V. Nanopoulos, Nucl. Phys: B106 (1976) 292.

[38] A. Djouadi, M. Spira e P. M. Zerwas, Phys. Lett. B264 (1991) 440.

[39] T. Han e S. Willenbrock, Phys. Lett. B273 (1991) 167.

[40] R. Cahn, proceedings da Ecole d'Ete de Physique des Particules, Centre de Recherches Nucléaires (CRN), Strasbourg, setembro de 1990.

[41] R. N. Cahn Nucl. Phys. B255 (1985) 341.

[42] C. von Weizacker, Z. Physics. 88 (1934) 612.

E. Williams, Phys. Rev. 45 (1934) 729.

[43] S. Dawson, Nucl. Phys. B249 (1985) 42.

[44] A. Donnachie, P. V. Landshoff Phys. Lett. B296 (1992) 227. 
[45] V. N. Gribov, Sov. Phys. JETP 26 (1968) 414;

M. Baker e K. A. Ter-Martirosyan, Phys. Rep. 28 (1976) 1;

M. Baker e L. McLerran, Proc. of International Conference on High Energy Physics, EPS, Palermo (Itália) 1975.

[46] A. Donnachie e P. V. Landshoff, Nucl. Phys. B244 (1984) 322.

[47] G. Ingelman e P. Schlein, Phys. Lett. B152 (1985) 256.

[48] A. Brandt et al., Phys. Lett. B297, (1992) 417.

[49] J. Collins, L. Frankfurt, e M. Strikman, Phys. Lett. B30 (1993) 161.

[50] K. Goulianos, Comments Nucl. Part. Phys. 17 (1987) 177.

[51] J. Pumplin, Phys. Rev. D8 (1973) 2899.

[52] U. P. Sukhatme e F. S. Henyey, Nucl. Phys. B108 (1976) 317; Nucl. Phys. B102 (1976) 275.

[53] P. Renton, Electroweak Interactions and Introduction to the Physics of Quarks and Leptons, Cambridge University Press, Cambridge (1990).

[54] Ta-Pei Cheng e Ling-Fong Li, Gauge Theory of Elementary Particle Physics, Clarendon Press, Oxford (1984).

[55] J. L. Cardy e G. A. Winbow, Phys. Lett. B52 (1974) 95.

[56] C. E. De Tar, S. D. Ellis e P. V. Landshoff Nucl. Phys. B87 (1975) 176.

[57] C. Collins, D. E. Soper e G. Sterman, Phys. Lett. B109 (1982) 388; Phys. Lett. B126 (1983) 275.

[58] G. T. Bodwin, Phys. Rev. D31 (1985) 2616; D34 (1986) 3932.

[59] R. Doria, J. Frenkel e J. C. Taylor, Nucl. Phys. B168 (1980) 93.

[60] J. C. Collins e D. E. Soper, Ann. Rev. Nucl. Part. Sci. 37 (1987) 383.

[61] O. Nachtmann, palestra proferida na 18a. Johns Hopkins Workshop, Florença, 1994; preprint HD-THEP-94-42. 
[62] S. Falciano et al., (NA10 coll.), Z. Phys. C31 (1986) 513;

M. Guanziroli et al., (NA10 coll), Z. Phys. C37 (1988) 545.

[63] H. Baer, F. Paige, S. Protopopescu e X. Tata, Florida State University preprint FSU-HEP-930329.

[64] T. Sjöstrand, Computer Physics Commun. 39 (1986) 347;

T. Sjöstrand e M. Bengtsson, Computer Physics Commun. 43 (1987) 367.

[65] V. A. Abramovskii, O. V. Kancheli e V. N. Gribov, Proceedings XVI International Conference on High Energy Physics, NAL (1972) 389.

[66] A. Krzywicki e B. Peterson, Phys. Rev. D6 (1972) 924;

J. Finkelstein e R. D. Peccei, Phys. Rev. D6 (1972) 2606;

F. Niedermayer, Nucl. Phys. B79 (1974) 355;

A. Casher, J. Kogut e L. Susskind, Phys. Rev. D10 (1974) 732.

[67] R. D. Field e R. P. Feynman, Nucl. Phys. B136 (1978) 1.

[68] K. Werner, Phys. Lett. B197 (1987) 225;

$Z$ Phys. C38 (1988) 193;

Nuclear Physics A525 (1991) 501C;

Phys. Rep. 232 (1993) 89.

[69] N. S. Amelin, et al. Sov. J. Nucl. Phys. 51 (1990) 535;

Sov. J. Nucl. Phys. 52 (1990) 362.

[70] P. Aurenche, F. W. Bopp, A. Capella, J. Kwiecinski, M. Maire, J. Ranft e J. Tran Thanh Van, Phys. Rev. D45 (1992) 92.

[71] P. Bruni e G. Ingelman, em preparação.

[72] H. U. Bengtsson e T. Sjöstrand, Computer Physics Commun. 46 (1987) 43.

[73] B. Andersson, G. Gustafson, G. Ingelman e T. Sjöstrand, Phys. Rep. 97 (1983) 31;

T. Sjöstrand, Nucl. Phys. B248 (1984) 469. 
[74] T. Sjöstrand, preprint CERN-TH.7112/93 (revisado em fevereiro de 1994).

[75] R. P. Feynman, Phys. Rev. D23 (1969) 1415;

Photon-Hadron Interactions, W. A. Benjamin, New York, 1972.

[76] G. 't Hooft, Nucl. Phys. B71 (1974) 461.

[77] G. Veneziano,Nucl. Phys. B74 (1974) 365;

Nucl. Phys. B117 (1976) 519.

[78] A. Capella, U. Sukhatme, C. -I. Tan e J. Tran Thanh Van, Phys. Rep. 236 (1994) 225.

[79] A. Capella, Proceedings of the Europhysics Study Conference, Erice, Itália, Março de 1981, ed. por R. T. Van de Walle; World Scientific Singapore.

[80] K. Hahn e J. Ranft, Phys. Rev. D41 (1990) 1463;

F. W. Bopp, A. Capella, J. Ranft e Tran Thanh Van, Z. Phys. C 51 (1990) 99.

[81] Z. Koba, H. B. Nielsen, P.Olsen, Nuclear Physics C 29 (1972) 201.

[82] K. Hahn, J. Ranft preprint KMU-HEP-89-04.

[83] J. Ranft preprint LNF-94/035(P) do Laboratori Nazionali di Frascati.

[84] Y. Dokshitzer, V. Khoze, S. Troyan, in Physics in Collision VI, Proceedings of the International Conference, Chicago, Illinois, 1986, editado por M. Derrick (World Scientific, Singapore, 1987), p. 365.

[85] R. N. Cahn e S. Dawson, Phys. Lett. B136 (1984) 196; erratum B138 (1984) 464;

S. Dawson Nucl. Phys. B249 (1984) 85;

G. Altarelli, B. Mele e F. Pitolli, Nucl. Phys. B287 (1987) 205.

[86] Y. L.Dokshitzer, V. A. Khoze, T. Sjostrand, Phys. Lett. B274, (1992) 116.

[87] T. Sjostrand e M. van Zijl, Phys. Rev. D36 (1987) 2019. 
[88] Conversa particular com T. Sjöstrand.

[89] J. D. Bjorken Phys. Rev. D47 (1993) 101.

[90] M. Block, F. Halzen, B. Margolis, Phys. Rev. D45 (1992) 839.

[91] E. Levin, Phys. Rev. D48 (1993) 2097.

[92] E. Gotsman, E. M. Levin e U. Maor, Phys. Lett. B309 (1993) 199.

[93] R. S. Fletcher e T. Stelzer, Phys. Rev. D48 (1993) 5162.

[94] G. Marchesini e B. R. Webber, Nucl. Phys. B310 (1988) 461

G. Marchesini et al. Comput. Phys. Commun. 67 (1992) 465.

[95] A. H. Mueller, Phys. Lett. B104 (1981) 161;

B. I. Ermolaev e V. S. Fadin, JETP Lett. 33 (1981) 269.

[96] J. Botts et al. (CTEQ-Collaboration), Phys. Lett. B304 (1993) 159.

[97] Yuan-Hann Chang e B. Zhou, preprint GEM MEMO PDT-BU-92-8.

[98] E. Eichten, I. Hinchliffe, K. Lane e C. Quigg, Rev. Mod. Phys. 56 (1984) 579

Rev. Mod. Phys. 58 (1985) 1065.

[99] M. Derrick et al. (ZEUS Collaboration) Phys. Lett. B315, 481 (1993).

J. Dainton (H1 Collaboration), RAL-94-012.

[100] Higgs Search with the GEM Detector, preprint GEM TN-93-373.

[101] V. Barger e R. J. N. Phillips, Conferência apresentada por V. Barger na VII Jorge André Swieca Summer School, Brasil, 1993.

[102] O. J. P. Éboli, T. L. Lungov e R. Zukanovich Funchal, Análise de Sinal e Background para Deteç̧ão de Leptoquarks Escalares no LHC, em preparação.

[103] V. Barger, R. J. N. Phillips e D. Zeppenfeld, Phys. Lett. B346 (1995) 106. 